\title{
A New Look at T Tauri Star Forbidden Lines: MHD-driven Winds from the Inner Disk
}

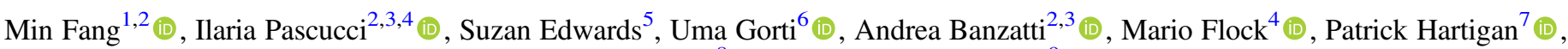 \\ Gregory J. Herczeg ${ }^{8}$ (D), and Andrea K. Dupree ${ }^{9}$ (i) \\ ${ }^{1}$ Department of Astronomy, University of Arizona, 933 North Cherry Avenue, Tucson, AZ 85721, USA \\ ${ }^{2}$ Earths in Other Solar Systems Team, NASA Nexus for Exoplanet System Science, USA \\ ${ }^{3}$ Department of Planetary Sciences, University of Arizona, 1629 East University Boulevard, Tucson, AZ 85721, USA \\ ${ }^{4}$ Max Planck Institute for Astronomy (MPIA), Königsthul 17, D-69117, Heidelberg, Germany \\ ${ }^{5}$ Five College Astronomy Department, Smith College, Northampton, MA 01063, USA \\ ${ }^{6}$ SETI Institute/NASA Ames Research Center, Mail Stop 245-3, Moffett Field, CA 94035-1000, USA \\ ${ }^{7}$ Department of Physics and Astronomy, Rice University, 6100 S. Main Street, Houston, TX 77005-1892, USA \\ Department of Physics and Astronomy, Rice University, 6100 S. Main Street, Houston, TX 77005-1892, USA
${ }^{8}$ Kavli Institute for Astronomy and Astrophysics, Peking University, Yiheyuan 5, Haidian Qu, 100871 Beijing, People's Republic of China \\ ${ }^{9}$ Harvard-Smithsonian Center for Astrophysics, Cambridge, MA 02138, USA \\ Received 2018 August 20; revised 2018 October 7; accepted 2018 October 8; published 2018 November 15
}

\begin{abstract}
Magnetohydrodynamic (MHD) and photoevaporative winds are thought to play an important role in the evolution and dispersal of planet-forming disks. We report the first high-resolution $\left(\Delta v \sim 6 \mathrm{~km} \mathrm{~s}^{-1}\right)$ analysis of $[\mathrm{S} \mathrm{II}] \lambda 4068$, [O I] $\lambda 5577$, and [O I] $\lambda 6300$ lines from a sample of $48 \mathrm{~T}$ Tauri stars. Following Simon et al. we decompose them into three kinematic components: a high-velocity component (HVC) associated with jets, and low-velocity narrow (LVCNC) and broad (LVC-BC) components. We confirm previous findings that many LVCs are blueshifted by more than $1.5 \mathrm{~km} \mathrm{~s}^{-1}$ and thus most likely trace a slow disk wind. We further show that the profiles of individual components are similar in the three lines. We find that most LVC-NC and LVC-BC line ratios are explained by thermally excited gas with temperatures between 5000 and $10,000 \mathrm{~K}$ and electron densities of $\sim 10^{7}-10^{8} \mathrm{~cm}^{-3}$. The HVC ratios are better reproduced by shock models with a pre-shock $\mathrm{H}$ number density of $\sim 10^{6}-10^{7} \mathrm{~cm}^{-3}$. Using these physical properties, we estimate $\dot{M}_{\text {wind }} / \dot{M}_{\text {acc }}$ for the LVC and $\dot{M}_{\text {jet }} / \dot{M}_{\text {acc }}$ for the HVC. In agreement with previous work, the mass carried out in jets is modest compared to the accretion rate. With the likely assumption that the LVC-NC wind height is larger than the LVC-BC, the LVC-BC $\dot{M}_{\text {wind }} / \dot{M}_{\text {acc }}$ is found to be higher than the LVC-NC. These results suggest that most of the mass loss occurs close to the central star, within a few au, through an MHD-driven wind. Depending on the wind height, MHD winds might play a major role in the evolution of the disk mass.
\end{abstract}

Key words: accretion, accretion disks - ISM: jets and outflows - magnetohydrodynamics (MHD) - protoplanetary disks - stars: pre-main sequence

Supporting material: extended figure

\section{Introduction}

Circumstellar disks form as a result of angular momentum conservation during the protostellar core collapse (Shu 1977) and play an important role in both star and planet formation. At early times, a significant fraction of the stellar mass is accreted through the disk, and what is not accreted or dispersed via other mechanisms provides the raw material to build planets. As such, it is important to understand how disks evolve and disperse.

In the current paradigm, there are three main stages of disk evolution and dispersal (e.g., Gorti et al. 2016; Ercolano \& Pascucci 2017 for recent reviews). For most of the disk lifetime (Stage 1), evolution is primarily set by accretion. Beyond a fraction of the radius where the sound speed equals the local Keplerian orbital speed $\left(\sim 2\right.$ au for $10^{4} \mathrm{~K}$ gas, and $\gtrsim 10-100$ au for $100-1000 \mathrm{~K}$ gas for a Sun-like star) gas becomes unbound, and a photoevaporative thermal wind can be established. When the disk accretion rate through this radius drops below the wind mass-loss rate, photoevaporation limits the supply of gas to the inner disk, a gap is formed (Stage 2), and the inner disk drains onto the star on the local viscous timescale — of order 100,000 yr. In the last stage (Stage 3), there is no accretion onto the star, and the disk is rapidly cleared from the inside out by stellar high-energy photons directly irradiating the outer disk.

Although photoevaporative winds are believed to play an important role in disk dispersal, large-scale jets/outflows, which contribute to significant mass loss at the earliest stages, are usually attributed to an origin in a magnetohydrodynamic (MHD) wind (e.g., Frank et al. 2014 for a recent review). Such winds can be effective in removing disk angular momentum and in facilitating accretion of disk gas onto the star (e.g., Pudritz et al. 2007; Shang et al. 2007).

Recently, significant theoretical effort has been devoted to understanding MHD disk winds and their relation to accretion and disk dispersal. It has long been realized that the inclusion of nonideal MHD effects suppresses magnetorotational instability (MRI) turbulence (e.g., Gammie 1996), and recent numerical simulations show that even a weak net vertical magnetic field, which could be, for example, leftover from the earlier stage of cloud collapse, can drive MHD winds (e.g., Suzuki \& Inutsuka 2009; Fromang et al. 2013). Local box simulations (e.g., Bai \& Stone 2013) and radially global, but vertically restricted, simulations (Gressel et al. 2015; Béthune et al. 2017) show that the launch of MHD winds is robust, can occur over the planet-forming region $(\sim 1-30 \mathrm{au})$, and can drive accretion at the observed levels. However, the predicted wind mass-loss rate, and hence the accretion rate, strongly depends on the magnetic field strength and how it evolves (e.g., Armitage et al. 2013; Bai \& Stone 2017), both of which are poorly constrained. Therefore, identifying diagnostics that trace disk winds and deriving wind mass-loss rates are important to 
understand not only how disks disperse but also how they evolve.

Low-excitation optical forbidden lines, especially from the [O I] $\lambda 6300$ transition, have long been used to study jets/ outflows from T Tauri stars (e.g., Edwards et al. 1987; Hartigan et al. 1995). Their line profiles typically present two distinct components: a high-velocity component (HVC), blueshifted by $30-200 \mathrm{~km} \mathrm{~s}^{-1}$ from the stellar velocity, and a low-velocity component (LVC), typically blueshifted by $\sim 5 \mathrm{~km} \mathrm{~s}^{-1}$. Spatially resolved observations have demonstrated that HVCs are formed in extended collimated jets (e.g., Bacciotti et al. 2000; Lavalley-Fouquet et al. 2000; Woitas et al. 2002), most likely linked to MHD winds (e.g., Ferreira et al. 2006).

Employing much higher spectral resolution than in previous studies $\left(\Delta v \sim 4 \mathrm{~km} \mathrm{~s}^{-1}\right.$ ), Rigliaco et al. (2013) found that the [O I] LVC itself can be described by the combination of a narrow component (NC) and a broad component (BC). More recently, Simon et al. (2016) confirmed this finding on a much larger sample of $33 \mathrm{~T}$ Tauri stars observed with Keck/HIRES $\left(\Delta v \sim 7 \mathrm{~km} \mathrm{~s}^{-1}\right)$. Using the line profiles and inclinations from resolved disk images, they inferred that most of the LVC-BC arises within $\sim 0.5$ au while most of the LVC-NC arises outside this radius. By combining measured velocities with line widths and disk inclinations, they also found that the LVC-BC tends to be narrower and more blueshifted for closer to face-on disks, as shown in some disk wind models (e.g., Alexander 2008). Finally, since the emitting region is within the photoevaporating radius even for $10,000 \mathrm{~K}$ gas, they could conclude that the LVC-BC traces an MHD disk wind. The origin of the LVC-NC remains unclear, as the inferred radial extent is consistent with a thermally driven photoevaporative wind but no trend between blueshifts and disk inclinations was seen (Simon et al. 2016).

An important step in computing wind mass-loss rates is to constrain the properties of the emitting gas associated with the wind. Ratios of lines tracing the same kinematic component can be employed for this task (e.g., Dougados et al. 2010). However, so far the only lines analyzed at similarly high spectral resolution to distinguish $\mathrm{BC}$ and $\mathrm{NC}$ are the [O I] $\lambda 6300$ and [O I] $\lambda 5577$. Assuming that the lines are thermally excited, their ratios constrain the range of temperature-electron densities for the emitting gas and imply higher electron density in the region traced by the LVC-BC for gas at similar temperatures (Simon et al. 2016). However, far-UV photodissociation of $\mathrm{OH}$ molecules can produce a similar range of [O I] ratios in much cooler ( $\sim 1000 \mathrm{~K})$ gas (see, e.g., Harich et al. 2000 and Gorti et al. 2011 for an application to disks). In this scenario of nonthermal emission, line ratios do not constrain the temperature and density of the gas. In order to estimate wind mass-loss rates, it is therefore necessary to first establish that the [O I] $\lambda 6300$ and [O I] $\lambda 5577$ emission is thermal. As first pointed out in Natta et al. (2014), the [S II] $\lambda 4068$ has a critical density very similar to the [O I] $\lambda 6300$ line and is likely to be thermally excited; hence, the [S II] and [O I] line profiles, as well as their line ratio, can be used to distinguish between thermal and nonthermal excitation.

In this work, we expand on previous high-resolution studies by analyzing the $[\mathrm{S} \mathrm{II}] \lambda 4068$, [O I] $\lambda 5577$, and [O I] $\lambda 6300$ from a sample of $48 \mathrm{~T}$ Tauri stars with the main goal of determining whether the [O I] emission is thermal or nonthermal. In a parallel work, we focus on the kinematic behavior of individual [O I] components to clarify the link between jets and winds (Banzatti et al. 2018). First, we describe our sample, observations, and data reduction (Section 2). Then, we present our analysis (Section 3), which includes line decomposition in HVC, LVC-BC, and LVC-NC following Simon et al. (2016). In Section 4 we show that the [S II] $\lambda 4068$, [O I] $\lambda 5577$, and $[\mathrm{O}$ I] $\lambda 6300$ line profiles are very similar within each kinematic component, strongly suggesting that the [O I] emission is thermally produced, and not the result of photodisocciation of $\mathrm{OH}$ in a cool gas. We use line ratios to constrain the properties of the emitting gas and compute wind mass-loss rates in Section 5. We also calculate mass accretion rates from several permitted lines and compare accretion versus mass-loss rates (Section 5). We discuss the implications of our results in Section 6 and summarize our findings in Section 7.

\section{Sample and Data Reduction}

\subsection{Sample}

Our sample comprises 48 young stars with disks; see Table 1. Most of them belong to the following five star-forming regions and associations: Taurus, Lupus I, Lupus III, $\rho$ Oph, and Corona Australis. The TW Hya and Upper Sco associations have an average age of 5-10 Myr (Fang et al. 2013b; Donaldson et al. 2016; Fang et al. 2017), while all other regions are younger, with ages 1-3 Myr (Luhman \& Rieke 1999; Luhman et al. 2009; Sicilia-Aguilar et al. 2011; Frasca et al. 2017). We retrieve Gaia Data Release 2 parallactic distances for 47 of them from the geometric distance table provided by Bailer-Jones et al. (2018). No parallax is reported for DP Tau; hence, we adopt the median distance of nearby Taurus members, which is $\sim 130 \mathrm{pc}$. With these new distances, SZ 102 is two times farther away than other Lupus members, while V853 Oph is $\sim 50$ pc closer than the $\rho$ Oph star-forming region. However, we note that both sources have high astrometric excess noise in Gaia DR2 (2.881 and 5.944 for SZ 102 and V853 Oph, respectively), indicating that their astrometric solution is unreliable (Lindegren et al. 2018). Therefore, for these two sources we take the mean distance to Lupus III and $\rho$ Oph. Using stellar members collected by Alcalá et al. (2017) for Lupus and by Manara et al. (2015) for $\rho$ Oph, we find 160 pc for SZ 102 and 138 pc for V853 Oph.

In addition to source distance and association, Table 1 also lists stellar spectral type, visual extinction $\left(A_{\mathrm{V}}\right)$, luminosity, radius, and mass $\left(L_{\star}, R_{\star}, M_{\star}\right)$, disk type and inclination $(i)$, and corresponding references. Note that stellar luminosities from the literature have been scaled to the new distances in our table. Stellar radii are calculated from the Stefan-Boltzmann equation, where the effective temperature is derived from the source spectral type according to the spectral type-effective temperature relation in Herczeg \& Hillenbrand (2014). Stellar masses are calculated from the luminosity and effective temperature using the nonmagnetic pre-main-sequence evolutionary tracks of Feiden (2016). Our sample covers a large range in spectral type (F7 to M4) and hence in stellar mass $\left(\sim 2.8\right.$ to $\left.\sim 0.2 M_{\odot}\right)$. Note that the two sources in Cygnus (V1057 Cyg and V1515 Cyg) are well-known FU Ori objects, young stars showing strong episodic accretion bursts (e.g., Audard et al. 2014).

On the disk type, we distinguish "full disks" from "transitional disks" (TDs). TDs are known to have reduced near- and midinfrared excess emission with respect to the median of $\mathrm{T}$ Tauri stars pointing to a dust-depleted inner region (e.g., Espaillat et al. 2014). Hence, we classify our sources by comparing the source spectral energy distribution (SED) to the median SEDs of classical T Tauri stars (CTTSs) of similar spectral type; see Appendix A for details. With our approach we identify 28 full 
Table 1

A List of the Sources in This Work, as well as Their Stellar and Disk Properties and the Used Photospheric Templates

\begin{tabular}{|c|c|c|c|c|c|c|c|c|c|c|c|c|c|c|c|}
\hline \multirow[t]{2}{*}{ ID } & \multirow[t]{2}{*}{ Name } & \multirow[t]{2}{*}{ Region } & \multirow{2}{*}{$\begin{array}{l}\text { Dist } \\
(\mathrm{pc})\end{array}$} & \multirow{2}{*}{$\begin{array}{l}\text { Spt } \\
\text { Type }\end{array}$} & \multirow{2}{*}{$\begin{array}{c}\log L_{\star} \\
\left(L_{\odot}\right)\end{array}$} & \multirow{2}{*}{$\begin{array}{c}A_{\mathrm{V}} \\
(\mathrm{mag})\end{array}$} & \multirow{2}{*}{$\begin{array}{c}R_{\star} \\
\left(R_{\odot}\right)\end{array}$} & \multirow{2}{*}{$\begin{array}{c}M_{\star} \\
\left(M_{\odot}\right)\end{array}$} & \multirow[t]{2}{*}{ Disk } & \multirow{2}{*}{$\begin{array}{c}i \\
(\mathrm{deg})\end{array}$} & \multirow[t]{2}{*}{ Reference } & \multirow{2}{*}{$\begin{array}{l}\mathrm{RV}(\text { Helio}) \\
\left(\mathrm{km} \mathrm{s}^{-1}\right)\end{array}$} & \multirow{2}{*}{$\begin{array}{l}\text { Correction } \\
\left(\mathrm{km} \mathrm{s}^{-1}\right)\end{array}$} & \multicolumn{2}{|c|}{ Photospheric Template } \\
\hline & & & & & & & & & & & & & & [S II] $\lambda 4068$ & {$[\mathrm{O} \mathrm{I}] \lambda 5577,[\mathrm{O} \mathrm{I}] \lambda 6300$} \\
\hline 1 & DP Tau & Taurus & 130 & M0.8 & -1.24 & 0.80 & 0.56 & 0.55 & Full & & 1 & $16.55 \pm 0.89$ & -0.64 & no correction & TWA 13 \\
\hline 2 & CX Tau & Taurus & 126.8 & M2.5 & -0.61 & 0.25 & 1.36 & 0.35 & $\mathrm{TD}$ & 61 & 1,9 & $19.15 \pm 1.31$ & -0.40 & $\ldots$ & TWA $8 A$ \\
\hline 3 & FP Tau & Taurus & 127.2 & M2.6 & -0.81 & 0.60 & 1.10 & 0.35 & TD & 66 & 1,9 & $16.90 \pm 2.11$ & -0.75 & TWA 8A & TWA 8A \\
\hline 4 & FN Tau & Taurus & 129.7 & M3.5 & -0.29 & 1.15 & 2.22 & 0.24 & Full & & 1 & $16.29 \pm 1.23$ & -0.09 & TWA 8A & TWA 8A \\
\hline 5 & V409 Tau & Taurus & 130.2 & M0.6 & -0.19 & 1.00 & 1.87 & 0.47 & Full & & 1 & $17.78 \pm 0.73$ & -0.49 & TWA 13 & TWA 13 \\
\hline 6 & BP Tau & Taurus & 127.7 & M0.5 & -0.40 & 0.45 & 1.45 & 0.54 & Full & 39 & 1,10 & $16.76 \pm 0.54$ & -0.43 & TWA 13 & V819 Tau \\
\hline 7 & DK Tau A & Taurus & 127.1 & K8.5 & -0.35 & 0.70 & 1.41 & 0.66 & Full & 26 & 1,11 & $16.60 \pm 0.42$ & -0.71 & TWA 13 & V819 Tau \\
\hline 8 & HN Tau A & Taurus & 133.3 & K3 & -0.81 & 1.15 & 0.63 & 0.69 & Full & 75 & 1,9 & $18.91 \pm 1.96$ & -0.46 & no correction & $\begin{array}{c}\text { 2MASS J15584772- } \\
1757595\end{array}$ \\
\hline 9 & UX Tau A & Taurus & 137.5 & K0 & 0.18 & 0.65 & 1.74 & 1.40 & $\mathrm{TD}^{\mathrm{a}}$ & 39 & 1,11 & $18.80 \pm 0.64$ & -0.44 & EPIC $212021375^{\mathrm{b}}$ & EPIC 203476597 \\
\hline 10 & GK Tau A & Taurus & 128.1 & K6.5 & -0.11 & 1.50 & 1.78 & 0.67 & Full & 71 & 1,9 & $18.52 \pm 0.64$ & -0.49 & HD 201092 & V819 Tau \\
\hline 11 & GI Tau & Taurus & 129.2 & M0.4 & -0.32 & 2.05 & 1.58 & 0.53 & Full & & 1 & $19.02 \pm 0.55$ & -0.40 & TWA 13 & V819 Tau \\
\hline 12 & DM Tau & Taurus & 143.5 & M3.0 & -0.87 & 0.10 & 1.05 & 0.31 & $\mathrm{TD}^{\mathrm{a}}$ & 34 & 1,11 & $19.64 \pm 1.50$ & -1.36 & TWA 8A & TWA 8A \\
\hline 13 & LkCa 15 & Taurus & 156.9 & K5.5 & -0.11 & 0.30 & 1.69 & 0.76 & $\mathrm{TD}^{\mathrm{a}}$ & 51 & 1,11 & $18.71 \pm 0.46$ & -0.55 & HD $36003^{b}$ & V819 Tau \\
\hline 14 & DS Tau & Taurus & 157.2 & M0.4 & -0.62 & 0.25 & 1.12 & 0.62 & Full & 71 & 1,9 & $16.51 \pm 0.97$ & -0.28 & TWA 13 & TWA 13 \\
\hline 15 & SZ 65 & Lupus & 153.4 & K6 & -0.03 & 0.80 & 1.90 & 0.68 & $\mathrm{TD}$ & 61 & 1,19 & $-2.85 \pm 0.90$ & -0.60 & HD $36003^{\mathrm{b}}$ & V819 Tau \\
\hline 16 & SZ 68A & Lupus & 152.1 & $\mathrm{~K} 2$ & 0.75 & 1.00 & 3.57 & 1.27 & Full & 33 & 1,19 & $-1.41 \pm 1.28$ & -0.74 & EPIC $212021375^{\mathrm{b}}$ & $\begin{array}{c}\text { 2MASS J15584772- } \\
1757595\end{array}$ \\
\hline 17 & SZ 73 & Lupus & 154.8 & K8.5 & -0.74 & 2.75 & 0.90 & 0.75 & Full & 50 & 1,12 & $-3.56 \pm 1.59$ & -1.10 & HD $201092^{\mathrm{b}}$ & V819 Tau \\
\hline 18 & HM Lup & Lupus & 154.1 & M2.9 & -0.78 & 0.60 & 1.16 & 0.32 & Full & 53 & 1,12 & $-1.62 \pm 1.82$ & -0.90 & TWA 8A & TWA 8A \\
\hline 19 & GW Lup & Lupus & 153.9 & M2.3 & -0.61 & 0.55 & 1.34 & 0.37 & Full & 40 & 1,12 & $-1.17 \pm 1.12$ & -0.77 & TWA 8A & TWA 8A \\
\hline 20 & GQ Lup & Lupus & 150.1 & K5.0 & -0.04 & 1.60 & 1.80 & 0.78 & Full & 60 & 1,13 & $-2.13 \pm 0.29$ & -1.00 & HD $36003^{b}$ & V819 Tau \\
\hline 21 & SZ 76 & Lupus & 157.5 & M3.2 & -0.69 & 0.90 & 1.33 & 0.28 & $\mathrm{TD}^{\mathrm{a}}$ & & 1 & $-1.14 \pm 2.16$ & -1.01 & TWA 8A & TWA 8A \\
\hline 22 & RU Lup & Lupus & 157.2 & K7.0 & 0.16 & 0.00 & 2.48 & 0.55 & Full & 3 & 2,19 & $-0.8 \pm 2$ & -0.98 & no correction & V819 Tau \\
\hline 23 & IM Lup & Lupus & 156.4 & K6.0 & 0.01 & 0.40 & 1.98 & 0.67 & $\mathrm{TD}$ & 48 & 1,19 & $-0.64 \pm 0.58$ & -1.18 & HD $36003^{\mathrm{b}}$ & V819 Tau \\
\hline 24 & RY Lup & Lupus & 156.6 & $\mathrm{~K} 2$ & 0.26 & 0.40 & 2.02 & 1.27 & Full & 68 & 3,22 & $-0.43 \pm 1.09$ & -0.90 & EPIC $212021375^{\mathrm{b}}$ & EPIC 203476597 \\
\hline 25 & SZ 102 & Lupus & 160 & $\mathrm{~K} 2$ & -2.02 & 0.70 & 0.15 & $\ldots$ & Full & 73 & 3,20 & $12 \pm 2$ & -1.82 & no correction & no correction \\
\hline 26 & SZ 111 & Lupus & 156.9 & M1.2 & -0.69 & 0.85 & 1.11 & 0.50 & $\mathrm{TD}^{\mathrm{a}}$ & 53 & 1,22 & $-0.16 \pm 0.74$ & -1.32 & HD $209290^{\mathrm{b}}$ & V819 Tau \\
\hline 27 & SZ 98 & Lupus & 154.3 & M0.4 & -0.49 & 1.25 & 1.30 & 0.58 & Full & 47 & 1,19 & $-0.32 \pm 0.70$ & -0.86 & TWA 13 & TWA 13 \\
\hline 28 & EX Lup & Lupus & 157.0 & M0 & -0.12 & 1.1 & 2.04 & 0.44 & Full & 38 & 3,28 & $2 \pm 1$ & -0.88 & no correction & V819 Tau \\
\hline 29 & As $205 \mathrm{~A}$ & $\rho$ Oph & 125.9 & K5 & 0.89 & 1.75 & 5.26 & 0.68 & Full & 25 & $1,4,14$ & $-5.31 \pm 0.55$ & -1.78 & HD $36003^{b}$ & V819 Tau \\
\hline 30 & DoAr 21 & $\rho$ Oph & 132.6 & G1 & 1.32 & 7.10 & 4.50 & 2.79 & $\mathrm{TD}^{\mathrm{a}}$ & & 1 & $-1.63 \pm 2.44$ & -1.46 & $\ldots$ & EPIC 203476597 \\
\hline 31 & DoAr 24E & $\rho$ Oph & 135.7 & K0 & 0.20 & 4.32 & 1.76 & 1.41 & Full & 20 & 5,30 & $-5.77 \pm 0.60$ & -1.66 & $\ldots$ & EPIC 203476597 \\
\hline 32 & DoAr 44 & $\rho \mathrm{Oph}$ & 144.3 & $\mathrm{~K} 2$ & -0.03 & 1.7 & 1.45 & 1.22 & $\mathrm{TD}^{\mathrm{a}}$ & 16 & 6,11 & $-4.50 \pm 0.53$ & -1.50 & EPIC $212021375^{\mathrm{b}}$ & $\begin{array}{c}\text { 2MASS J15584772- } \\
1757595\end{array}$ \\
\hline 33 & $\mathrm{EM}^{*} \mathrm{SR} 21 \mathrm{~A}$ & $\rho$ Oph & 136.8 & F7 & 0.98 & 6.2 & 2.62 & 1.79 & $\mathrm{TD}^{\mathrm{a}}$ & 18 & $1,11,15,16$ & $-5.66 \pm 3.65$ & -1.41 & HIP $42106^{\mathrm{b}}$ & HIP $42106^{\mathrm{b}}$ \\
\hline 34 & V853 Oph & $\rho \mathrm{Oph}$ & 138 & M2.5 & -0.30 & 0.14 & 1.95 & 0.32 & Full & 54 & 5,11 & $-5.8 \pm 1.10$ & -1.96 & TWA 8A & TWA 8A \\
\hline 35 & RNO 90 & $\rho$ Oph & 115.7 & G8 & 0.42 & 4.3 & 2.01 & 1.68 & Full & 37 & 17,30 & $-9.11 \pm 1.09$ & -1.70 & $\mathrm{KW} 27^{\mathrm{b}}$ & EPIC 203476597 \\
\hline 36 & V2508 Oph & $\rho$ Oph & 122.7 & K7 & -0.13 & 1.7 & 1.78 & 0.63 & Full & 41 & 17,11 & $-7.62 \pm 0.79$ & -1.94 & HD $201092^{\mathrm{b}}$ & V819 Tau \\
\hline 37 & V1121 Oph & $\rho \mathrm{Oph}$ & 119.7 & K4 & -0.06 & 1.08 & 1.62 & 0.96 & Full & 31 & 5,11 & $-6.27 \pm 0.32$ & -1.78 & HD $36003^{b}$ & TWA 9A \\
\hline 38 & $\begin{array}{c}\text { RX J1842.9 } \\
-3532\end{array}$ & $\begin{array}{l}\text { Corona } \\
\text { Australis }\end{array}$ & 152.2 & K3 & -0.23 & 0.6 & 1.24 & 1.07 & $\mathrm{TD}^{\mathrm{a}}$ & 54 & 1,18 & $-0.92 \pm 0.53$ & -1.31 & HD $36003^{b}$ & $\begin{array}{c}\text { 2MASS J15584772- } \\
1757595\end{array}$ \\
\hline 39 & $\begin{array}{c}\text { RX J1852.3 } \\
-3700\end{array}$ & $\begin{array}{l}\text { Corona } \\
\text { Australis }\end{array}$ & 144.1 & K4 & -0.40 & 0.25 & 1.10 & 0.96 & $\mathrm{TD}^{\mathrm{a}}$ & 16 & 1,18 & $0.64 \pm 0.53$ & -1.16 & HD $36003^{b}$ & $\begin{array}{c}\text { 2MASS J15584772- } \\
1757595\end{array}$ \\
\hline 40 & VV CrA & $\begin{array}{l}\text { Corona } \\
\text { Australis }\end{array}$ & 146.6 & K7 & 0.32 & 3.95 & 2.99 & 0.53 & Full & 49 & 1,21 & $-1.0 \pm 1$ & -1.39 & no correction & V819 Tau \\
\hline
\end{tabular}




\begin{tabular}{|c|c|c|c|c|c|c|c|c|c|c|c|c|c|c|c|}
\hline \multirow{2}{*}{ ID } & \multirow{2}{*}{ Name } & \multirow{2}{*}{ Region } & \multirow{2}{*}{$\begin{array}{l}\text { Dist } \\
(\mathrm{pc})\end{array}$} & \multirow{2}{*}{$\begin{array}{l}\text { Spt } \\
\text { Type }\end{array}$} & \multirow{2}{*}{$\begin{array}{c}\log L_{\star} \\
\left(L_{\odot}\right)\end{array}$} & \multirow{2}{*}{$\begin{array}{c}A_{\mathrm{V}} \\
\text { (mag) }\end{array}$} & \multirow{2}{*}{$\begin{array}{c}R_{\star} \\
\left(R_{\odot}\right)\end{array}$} & \multirow{2}{*}{$\begin{array}{c}M_{\star} \\
\left(M_{\odot}\right) \\
\end{array}$} & \multirow{2}{*}{ Disk } & \multirow{2}{*}{$\begin{array}{c}i \\
(\operatorname{deg})\end{array}$} & \multirow{2}{*}{ Reference } & \multirow{2}{*}{$\begin{array}{l}\mathrm{RV}(\text { Helio }) \\
\left(\mathrm{km} \mathrm{s}^{-1}\right)\end{array}$} & \multirow{2}{*}{$\begin{array}{l}\text { Correction } \\
\left(\mathrm{km} \mathrm{s}^{-1}\right)\end{array}$} & \multicolumn{2}{|c|}{ Photospheric Template } \\
\hline & & & & & & & & & & & & & & [S II] $\lambda 4068$ & {$[\mathrm{O}$ I] $\lambda 5577,[\mathrm{O}$ I] $\lambda 6300$} \\
\hline 41 & $\mathrm{SCrA} A+\mathrm{B}$ & $\begin{array}{l}\text { Corona } \\
\text { Australis }\end{array}$ & 150.3 & K6 & 0.24 & 0.5 & 2.58 & 0.61 & Full & 10 & 8,30 & $1.41 \pm 2.42$ & -1.00 & no correction & $\begin{array}{c}\text { 2MASS J15584772- } \\
1757595\end{array}$ \\
\hline 42 & TW Hya & TWA & 59.8 & M0.5 & -0.63 & 0.00 & 1.11 & 0.61 & $\mathrm{TD}^{\mathrm{a}}$ & 7 & 1,11 & $13.55 \pm 0.34$ & -1.24 & TWA 13 & V819 Tau \\
\hline 43 & TWA 3A & TWA & 36.4 & M4.1 & -1.19 & 0.05 & 0.85 & 0.16 & $\mathrm{TD}$ & & 1 & $11.84 \pm 2.52$ & -0.83 & TWA 7 & TWA 7 \\
\hline 44 & V1057 Cyg ${ }^{\mathrm{c}}$ & Cygnus & 897.7 & $\sim \mathrm{G} 0 ?$ & 2.47 & 4.63 & & & Full & & 23,17 & $-14.3 \pm 1$ & -1.17 & no correction & EPIC 203476597 \\
\hline 45 & V1515 $\mathrm{Cyg}^{\mathrm{c}}$ & Cygnus & 980.8 & $\sim \mathrm{G} 3 ?$ & 2.01 & 2.93 & & & Full & & $24,25,17$ & $-11.13 \pm 0.53$ & -1.40 & no correction & $\begin{array}{c}\text { 2MASS J15584772- } \\
1757595\end{array}$ \\
\hline 46 & HD $143006^{\mathrm{a}}$ & Upper Sco & 165.5 & G3 & 0.50 & 0.45 & 1.81 & 1.52 & $\mathrm{TD}^{\mathrm{a}}$ & $\sim 28$ & $1,17,29$ & $-0.19 \pm 0.21$ & -1.30 & $\mathrm{KW} 27^{\mathrm{b}}$ & KW54 $1^{\mathrm{b}}$ \\
\hline 47 & DI Cep & Cepheus & 430.0 & G8 & 0.85 & 0.25 & 3.27 & 2.28 & Full & & 26,17 & $-7.00 \pm 0.56$ & -2.36 & $\mathrm{KW} 27^{\mathrm{b}}$ & EPIC 203476597 \\
\hline 48 & As 353A & LDN 673 & 404.0 & K5 & 0.51 & 0.00 & 3.50 & 0.60 & Full & & 27,17 & $-7.87 \pm 0.60$ & -1.10 & no correction & HBC 427 \\
\hline
\end{tabular}

Notes.

${ }^{\text {a }}$ Listed as the TDs in van der Marel et al. (2016).

${ }^{\mathrm{b}}$ Main-sequence templates.

${ }^{\mathrm{c}}$ The stellar masses and radii for the two FU Ori are unknown since their spectral types are very uncertain.

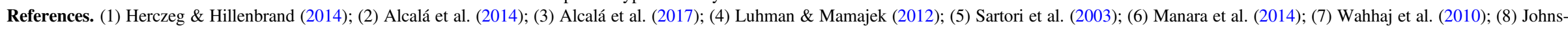

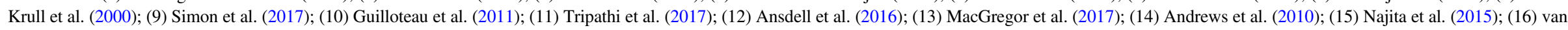

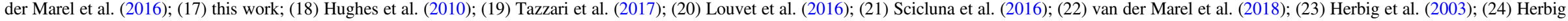
(1977); (25) Herbig \& Bell (1988); (26) Cohen \& Kuhi (1979); (27) Rice et al. (2006); (28) Hales et al. (2018); (29) Lazareff et al. (2017); (30) Pontoppidan et al. (2011). 
disks and 15 TDs. The five disks we could not classify are around $\mathrm{G}$ and $\mathrm{F}$ stars. As these stars are rare and their disks disperse faster (e.g., Kennedy \& Kenyon 2009), we simply lack a median SED for this spectral type range. For SR 21A and HD 143006 we adopt the literature classification of TDs (Najita et al. 2015; van der Marel et al. 2016). As there is no hint of reduced infrared emission in the SED of the other three G-type stars (V1057 Cyg, V1515 Cyg, and DI Cep), we classify their disks as full. Recently, van der Marel et al. (2016) used color criteria and SED modeling to identify a large sample of TD candidates in nearby star-forming regions. Of the 14 sources in common, 13 are classified in the same way. Only FN Tau is classified as full disk here but listed as TD in van der Marel et al. (2016). Note, however, that van der Marel et al. (2016) estimate an uncertainty on the cavity size that is as large as the cavity itself; hence, FN Tau might actually have a full disk as we adopt here.

\subsection{Observations and Data Reduction}

Our targets were observed during two nights, 2008 January 23 and May 23, through the program C199Hb (PI: Greg J. Herczeg). The Keck/HIRES spectrograph (Vogt et al. 1994) was used with the $\mathrm{C} 1$ decker and a 0 !" $861 \times 7^{\prime \prime}$ slit, resulting in a nominal resolution of 48,000. The sources were observed with the kv380 filter, wavelength coverage $3900-8500 \AA$, during the night of January 23 and with the wg335 filter, wavelength coverage $3480-6310 \AA$, during the night of May 23. In addition to the science targets, three telluric standards at least were observed each night. These standards are used to correct for telluric features (Section 3.1) near the [O I] $\lambda 6300$ line, as well as for flux calibration (Appendix B).

The raw data were reduced using the Maunakea Echelle Extraction (MAKEE) pipeline written by Tom Barlow. ${ }^{10}$ MAKEE is designed to run noninteractively using a set of default parameters and carry out bias subtraction, flat-fielding, and spectral extraction, including sky subtraction and wavelength calibration with ThAr calibration lamps. The wavelength calibration is performed in air by setting the keyword "novac" in the pipeline inputs. Heliocentric correction is also applied to the extracted spectra.

\subsection{Stellar Radial Velocity}

The stellar radial velocity (RV) is derived by crosscorrelating each star optical spectrum with the synthetic spectrum of a star that has the same effective temperature. The grid of model spectra is from Husser et al. (2013) for a solar abundance and surface gravity $\log g=4.0$. The model spectrum is first degraded to match the spectral resolution of HIRES, rotationally broadened to match the source photospheric features, and "veiled" by adding a constant flux value to account for the fact that photospheric lines from accreting $\mathrm{T}$ Tauri stars are less deep than those of non-accreting stars of the same spectral type. The cross-correlation is carried out separately for each echelle order that does not have strong emission lines or is highly veiled. Hence, the total number of available orders varies from source to source, but it is at least 5 , except for RU Lup, EX Lup, and V1057 Cyg. For RU Lup, we only detect photospheric features near the [O I] $\lambda 6300$ line and use them to estimate the stellar RV. For EX Lup, we detect photospheric features within 5733-5745 $\AA$ and 5936-5948

\footnotetext{
${ }^{10}$ http://www2.keck.hawaii.edu/inst/common/makeewww/index.html
}

and the cross-correlation is performed within these wavelength ranges. The stellar RV of V1057 Cyg is obtained by crosscorrelating the observed spectrum with the model spectrum within 5556-5580 $\mathrm{A}$ and $5645-5673 \AA$. Table 1 provides the stellar RV and associated uncertainty computed as the mean and standard deviation of the RVs obtained from individual orders.

SZ 102 and VV CrA have highly veiled spectra with strong emission lines; hence, no HIRES order can be used to derive their RVs. For these two sources, we download archival ESO Phase 3 X-Shooter VIS spectra, which are wavelength and flux calibrated and have a resolution of $\sim 17,400$ between 5500 and $10000 \AA$. For VV CrA, we derive its RV by fitting its Li I $\lambda 6708$ absorption line with a Gaussian function as in Pascucci et al. (2015). For SZ 102, we cannot clearly identify the Li I $\lambda 6708$ absorption line; hence, we derive its RV by crosscorrelating the observed spectrum with the model spectrum within 7485-7530, $8020-8112 \AA$, and 8762-8845 $\AA$ where we can identify photospheric absorption lines. The RVs of these two sources are also listed in Table 1.

Note that synthetic spectra are only used for the computation of stellar RVs. As discussed in the next section, photospheric subtraction is carried out mostly with the observed spectra of weak-line T Tauri stars (WTTSs). We do not have WTTS templates for 18 sources in the [S II] $\lambda 4068$ setting and for one source, SR 21A (F7), around the [O I] $\lambda 6300$ and the [O I] $\lambda 5577$ settings; hence, we used the main-sequence (MS) templates in these instances (see Table 1).

Arc calibration frames for the absolute wavelength solution were taken only at the beginning of each night. Therefore, systematic shifts on the wavelength solution are possible when moving the telescope. We assess these possible shifts by crosscorrelating the telluric lines with the model atmospheric transmission curve for the Keck Observatory, which is calculated with TAPAS (a web-based service of atmospheric transmission computation for astronomy; see Bertaux et al. 2014). We find that the wavelength calibration tends to be redshifted by $\sim 0.5-2 \mathrm{~km} \mathrm{~s}^{-1}$. In Table 1 , we list the RV correction for each source. Except for SZ 102 and VV CrA, whose RVs are estimated from X-Shooter spectra, the RVs of all other sources need to be corrected by adding the values in the "Correction" column to their RVs. However, the corrections only affect the heliocentric RV derived from the HIRES spectra. They do not change the relative shifts between the stellar photospheric absorption features and the forbidden lines, which are the focus of this paper. We list these corrections in case one wants to compare the RVs calculated here with those obtained via other methods.

\section{Line Profiles and Classification}

\subsection{Corrected Line Profiles and Detection Rates}

To produce corrected line profiles, we first remove any telluric absorption and then subtract the photospheric features near the lines. Telluric removal is only necessary for the [O I] $\lambda 6300$ line and is achieved with the procedure summarized in the HIRES Data Reduction manual ${ }^{11}$ and using the telluric standards acquired in the two nights.

After telluric removal, we subtract the stellar photosphere following Hartigan et al. (1989). In short, we select a

\footnotetext{
${ }^{11}$ See the description in https://www2.keck.hawaii.edu/inst/common/makee www/Atmosphere/index.html.
} 

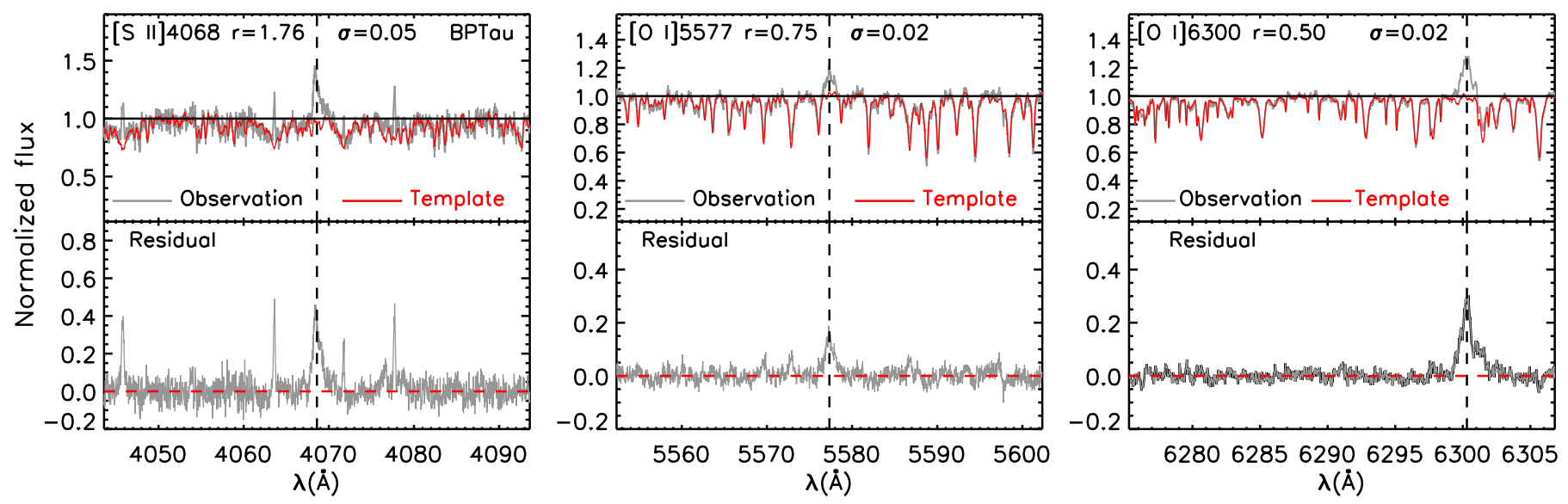

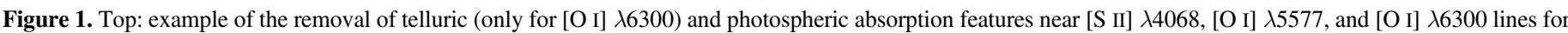

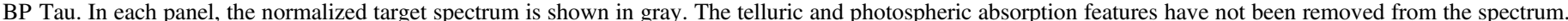

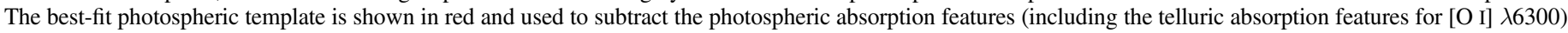
from the target spectrum. Bottom: final corrected line profiles of [S II] $\lambda 4068$, [O I] $\lambda 5577$, and [O I] $\lambda 6300$ of BP Tau.

photospheric standard with a spectral type similar to the target. We then broaden (through the rotation velocity $V \sin i$ ), veil (parameter $r_{\lambda}$ ), and shift in velocity its spectrum to best match the target's photospheric lines. Veiling is defined as the ratio of the excess $\left(F_{\text {excess }}\right)$ to the photospheric flux $\left(F_{\text {phot }}\right)$, $r_{\lambda}=F_{\text {excess }} / F_{\text {phot }}$, and it is used to mimic the filling in of the photospheric lines due to the excess emission from the accretion shocks. The best-fit photospheric spectrum is the one that minimizes the $\chi^{2}$, defined as $\chi^{2}=\sum\left(F_{\text {target }}-F_{\text {Standard }}\right)^{2}$. Corrected line profiles are produced by subtracting the best-fit photospheric spectra from the target spectra. We do not apply any photospheric subtraction on extremely veiled spectra that have no photospheric features, e.g., several of the spectra in the [S II] $\lambda 4068$ setting. Figure 1 shows an example of the technique described here, including telluric removal near the [O I] $\lambda 6300$ line (right panel). Table 1 lists the photospheric standards used for individual lines.

Overall, we detect the $[\mathrm{O} \mathrm{I}] \lambda 6300,[\mathrm{O} \mathrm{I}] \lambda 5577$, and [S II] $\lambda 4068$ lines from 45, 26, and 22 sources, respectively. ${ }^{12}$ Hence, the detection rate is $94 \%, 54 \%$, and $49 \%$ in these lines. ${ }^{13}$ A total of 19 sources have detections in all three lines.

\subsection{Line Decomposition}

As recently shown in Simon et al. (2016), oxygen forbidden lines can be well reproduced by the superimposition of a few Gaussian profiles, presumably tracing different kinematic components. Figure 2 compares the [S II] $\lambda 4068$, [O I] $\lambda 5577$, and $[\mathrm{OI}] \lambda 6300$ emission lines from DP Tau, one of the sources with the highest signal-to-noise ratio $(\mathrm{S} / \mathrm{N})$ spectra. Note how an NC centered at the stellar velocity is present in all lines (panel (a)). Broader, higher-velocity emission is also apparent in the three tracers (panel (b)).

Motivated by the similarity of the profiles in the high-S/N spectrum of DP Tau and following Simon et al. (2016), we use a combination of Gaussians to decompose the $[\mathrm{S} \mathrm{II}] \lambda 4068$,

\footnotetext{
${ }^{12}$ CX Tau, DoAr 21, and DoAr 24E are so faint at short wavelengths that their [S II] $\lambda 4068$ spectra cannot be extracted with the MAKEE pipeline. SR 21A shows only marginal detections in the [O I] $\lambda 6300$ line; see Figure 19. Of the three sources (SZ 68A, DoAr 21, and DoAr 24E) without [O I] $\lambda 6300$ detection, one is a TD and two are full disks; all have spectral types of early $\mathrm{K}$ to $\mathrm{G}$. A detailed discussion of these sources is presented in Appendix C.

${ }^{13}$ For the [S II] $\lambda 4068$ line, the three sources without extracted spectra have been excluded when calculating the detection rates.
}

[O I] $\lambda 5577$, and [O I] $\lambda 6300$ lines. To find the minimum number of Gaussians that describes the observed profile, we use an iterative approach based on the IDL procedure mpfitfun. We start by fitting each profile with one Gaussian and compute the residual spectrum by subtracting the best fit from the original profile. If the rms of the residual is higher than that of the original spectrum next to the line of interest (by more than $\sim 2 \sigma$ ), we add another Gaussian and refit the original profile simultaneously with two Gaussians. We recompute the residual spectrum and add an extra Gaussian until the rms of the residual is within $2 \sigma$ of the rms of the original spectrum.

Table 2 lists the parameters resulting from the decomposition of each individual line, while Figure 3 shows examples of the best-fit Gaussian profiles for three sources. All other fits are shown in Appendix D, Figure 19. Seven sources (HN Tau A, RU Lup, EX Lup, As 205A, VV CrA, SCrA A+B, and As 353A) have [S II] $\lambda 4068$ lines highly contaminated by $\mathrm{Fe}$ I emission. In addition, the [O I] $\lambda 5577$ line from RU Lup is also affected by Fe I emission. We decontaminate these line profiles before line decomposition as discussed in detail in Appendix E. Note that the $[\mathrm{S} \mathrm{II}] \lambda 4068$ profiles from EX Lup, As 205A, VV CrA, and As 353A are not well recovered at velocities more blueshifted than $\sim 200 \mathrm{~km} \mathrm{~s}^{-1}$. Hence, the most blueshifted HVC [S II] $\lambda 4068$ component for each of these sources is marked as unreliable in Table 2 and will not be used in the following analysis.

Table 2 also lists upper limits for undetected lines. For sources with detections in the [O I] $\lambda 6300$ line, the upper limits on the other lines are computed assuming that they share the [O I] $\lambda 6300$ profile and have peaks of $3 \times \mathrm{rms}$, where the $\mathrm{rms}$ is calculated from the standard deviation of the continuum near the line. For sources with nondetections in all the lines, the upper limits are calculated assuming a Gaussian profile with the FWHM of an unresolved line $\left(\sim 6.3 \mathrm{~km} \mathrm{~s}^{-1}\right)$ and a peak of $3 \times \mathrm{rms}$.

\subsection{Preliminary Classification}

Different approaches have been used in the literature to separate the LVC from the HVC (e.g., Hartigan et al. 1995; Natta et al. 2014). As our spectra have a similar resolution to those in Simon et al. (2016), we use their criteria for a preliminary classification; see the second-to-last column of 


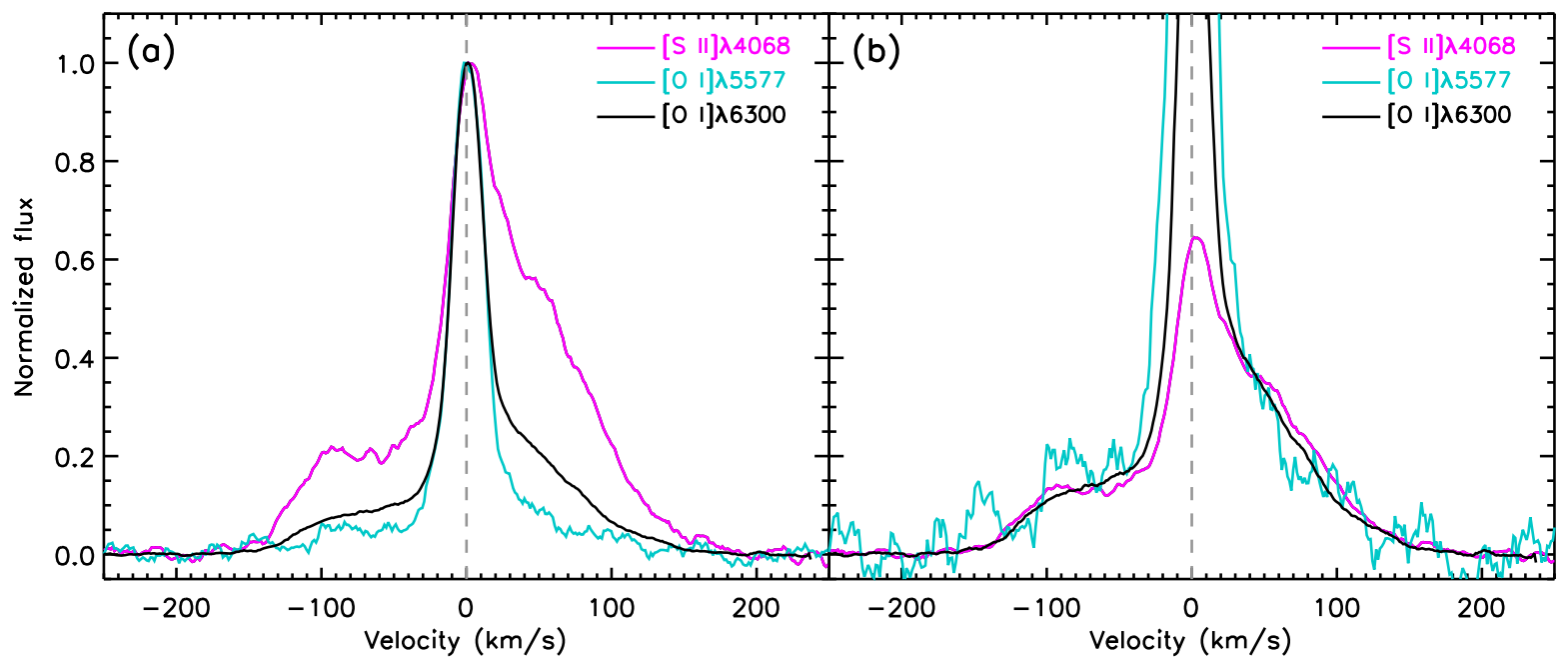

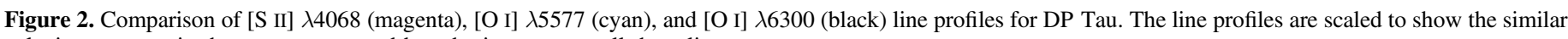
velocity structure in the narrow core and broad wings among all three lines.

Table 2. In short, a component is called LVC (HVC) if the absolute value of the Gaussian velocity centroid is smaller (larger) than $30 \mathrm{~km} \mathrm{~s}^{-1}$. Within the LVC, an LVC-BC has an FWHM $>40 \mathrm{~km} \mathrm{~s}^{-1}$, while an LVC-NC is narrower. As a consequence, even lines that can be fitted with one Gaussian fall into one of these categories (see also Simon et al. 2016 and McGinnis et al. 2018, but Banzatti et al. 2018 for an independent treatment of single components).

Based on this preliminary classification, we find that $\mathrm{HVCs}$ and LVCs are often present in the three line profiles but with different contributions: in the [S II] $\lambda 4068$ line the HVC tends to be more prominent relative to the $\mathrm{LVC}$ than in the [O I] $\lambda 6300$ and [O I] $\lambda 5577$ lines, the LVC dominates the [O I] $\lambda 5577$ profile, while both an HVC and an LVC are seen in the [O I] $\lambda 6300$ profile. Two extreme cases are worth discussing. The [O I] $\lambda 6300$ and [S II] $\lambda 4068$ profiles of FN Tau are the most complex in our sample, with one LVC and three HVCs, while its [O I] $\lambda 5577$ line only presents one LVC. For SZ 73, the [S II] $\lambda 4068$ and [O I] $\lambda 5577$ lines only have one component, classified as $\mathrm{HVC}$ and LVC, respectively, while its [O I] $\lambda 6300$ shows both one LVC and one HVC.

The above classification cannot be taken too rigidly, as there is some overlap in line widths between BC and NC (Banzatti et al. 2018), and HVCs could show small blueshifts in highly inclined systems and be wrongly classified as LVCs when adopting the sharp velocity boundary of $30 \mathrm{~km} \mathrm{~s}^{-1}$ utilized here. In the next section we will use line ratios to test and further refine our classification.

\subsection{Refined Classification}

Ratios of lines that trace the same kinematic component can be used to constrain the properties of the emitting gas. Simon et al. (2016) have already shown that the [O I] $\lambda 6300$ and [O I] $\lambda 5577$ lines have very similar LVC profiles (e.g., their Figure 15 and best-fit parameters in Table 5). Section 4.1 will illustrate that, within a specific kinematic component, the similarity extends to the [S II] $\lambda 4068$ line. Hence, here we use the ratios of these three forbidden lines to test whether different kinematic components separate out and, if so, further refine our preliminary classification.

Figure 4 shows line ratios (left panel) or upper limits (right panel) for all 45 sources that are detected in the [O I] $\lambda 6300$, the brightest of our forbidden lines. HVCs (green circles) have a combination of higher [S II] $\lambda 4068 /[\mathrm{O}$ I] $\lambda 6300$ (hereafter SII40/OI63) and lower [O I] $\lambda 5577 /[\mathrm{O}$ I] $\lambda 6300$ (hereafter OI55/63) ratios than LVCs. This is best illustrated by the mean and standard deviation of different component line ratios, which are shown as dotted ellipses in both panels. Note that these values are computed from our preliminary classification and only from sources with at least two detected forbidden lines that are used to calculate line ratios.

The left panel of Figure 4 reveals four possible LVCs with line ratios more compatible with HVCs (circles surrounded by green squares: one LVC-BC from DP Tau (ID 1) and HN Tau A (ID 8), and one LVC-NC and one LVC-BC from SZ 102 (ID 25). Interestingly, HN Tau A and SZ 102 have close to edge-on disks $\left(i>70^{\circ}\right)$. Thus, it is very likely that, owing to projection effects, their HVCs have centroids $<30 \mathrm{~km} \mathrm{~s}^{-1}$ and have been erroneously categorized as LVCs by our preliminary classification scheme. Although the disk inclination of DP Tau is not known, the star is about an order of magnitude underluminous compared to other Taurus members of the same spectral type (Herczeg \& Hillenbrand 2014), pointing to obscuration from a highly inclined disk (see discussions on such underluminous young stars in Fang et al. 2009, 2013b). In addition, the [O I] $\lambda 6300$ and [S II] $\lambda 4068$ LVC-BC centroids are close to our $30 \mathrm{~km} \mathrm{~s}^{-1} \mathrm{HVC} / \mathrm{LVC}$ boundary. Thus, we reclassify all four LVCs as HVCs; see the last column of Table 2.

Figure 4 also shows the expected SII40/OI63 and OI55/63 ratios for gas at $10^{4} \mathrm{~K}$, where collisions with electrons (densities $10^{5.8}-10^{8.5} \mathrm{~cm}^{-3}$ ) excite the upper level of the transitions (e.g., Natta et al. 2014; see also Section 4.2.1). Most HVCs have SII40/OI63 ratios higher than the model-predicted ones, while most LVCs have lower ratios. We also note that one LVC-BC from As 353A (ID 48; right panel) shows higher SII40/OI63 ratios than the model-predicted ones, similar to HVCs. This, combined with possible Fe I contamination in the [S II] $\lambda 4068$ transition (see Appendix E), leads us to mark the LVC-BC as suspicious; see the last column of Table 2.

Following this refined classification, we find that $51 \%$ (23/45) of the sources with [O I] $\lambda 6300$ detection have an HVC, while $84 \%(38 / 45)$ show an LVC. Furthermore, $31 \%(14 / 45)$ of the sample presents an LVC-BC, while 33\% (15/45) has an 
Table 2

Parameters for Line Decomposition of [S II] $\lambda 4068$, [O I] $\lambda 5577$, and [O I] $\lambda 6300$ Line Profiles

\begin{tabular}{|c|c|c|c|c|c|c|c|c|c|c|c|c|c|c|c|}
\hline \multirow[b]{2}{*}{ ID } & \multirow[b]{2}{*}{ Name } & \multicolumn{4}{|c|}{ [S II] $\lambda 4068$} & \multicolumn{4}{|c|}{ [O I] $\lambda 5577$} & \multicolumn{4}{|c|}{ [O I] $\lambda 6300$} & \multirow[b]{2}{*}{$\begin{array}{l}\text { Preliminary } \\
\text { Class }\end{array}$} & \multirow[b]{2}{*}{$\begin{array}{c}\text { Refined } \\
\text { Class }\end{array}$} \\
\hline & & $\begin{array}{l}\text { FWHM } \\
\left(\mathrm{km} \mathrm{s}^{-1}\right)\end{array}$ & $\begin{array}{c}v_{\mathrm{c}} \\
\left(\mathrm{km} \mathrm{s}^{-1}\right)\end{array}$ & $\begin{array}{l}\mathrm{EW} \\
(\AA)\end{array}$ & $\begin{array}{c}\log L_{4069} \\
\left(L_{\odot}\right)\end{array}$ & $\begin{array}{l}\text { FWHM } \\
\left(\mathrm{km} \mathrm{s}^{-1}\right)\end{array}$ & $\begin{array}{c}v_{\mathrm{c}} \\
\left(\mathrm{km} \mathrm{s}^{-1}\right)\end{array}$ & $\begin{array}{l}\text { EW } \\
(\AA)\end{array}$ & $\begin{array}{c}\log L_{5577} \\
\left(L_{\odot}\right)\end{array}$ & $\begin{array}{c}\text { FWHM } \\
\left(\mathrm{km} \mathrm{s}^{-1}\right)\end{array}$ & $\begin{array}{c}v_{\mathrm{c}} \\
\left(\mathrm{km} \mathrm{s}^{-1}\right)\end{array}$ & $\begin{array}{l}\mathrm{EW} \\
(\AA)\end{array}$ & $\begin{array}{c}\log L_{6300} \\
\left(L_{\odot}\right)\end{array}$ & & \\
\hline \multirow[t]{3}{*}{1} & DP Tau & 62.8 & -91.9 & 1.01 & -5.29 & 45.4 & -90.6 & 0.08 & -6.32 & 64.4 & -89.3 & 0.89 & -5.24 & HVC-B & \\
\hline & & 115.6 & 31.2 & 5.92 & -4.52 & 116.7 & 13.6 & 0.47 & -5.54 & 111.8 & 23.5 & 6.03 & -4.41 & LVC-BC & HVC-R \\
\hline & & 27.3 & 2.4 & 1.23 & -5.20 & 24.4 & 0.4 & 0.60 & -5.44 & 22.5 & 0.8 & 3.99 & -4.59 & LVC-NC & \\
\hline 2 & CX Tau & $\ldots$ & $\ldots$ & $\ldots$ & $\ldots$ & $\ldots$ & $\ldots$ & $\ldots$ & $<-6.52$ & 27.9 & -2.6 & 0.05 & -6.40 & LVC-NC & \\
\hline 3 & FP Tau & $\ldots$ & $\ldots$ & $\ldots$ & $<-5.51$ & 84.6 & 1.4 & 0.14 & -6.14 & 66.0 & 1.1 & 0.38 & -5.68 & LVC-BC & \\
\hline \multirow[t]{4}{*}{4} & FN Tau & 13.8 & -44.1 & 0.09 & -6.18 & $\cdots$ & $\cdots$ & $\cdots$ & $<-6.47$ & 10.6 & -43.0 & 0.03 & -6.25 & HVC-B & \\
\hline & & 15.3 & -117.8 & 0.14 & -5.99 & $\cdots$ & $\cdots$ & $\ldots$ & $<-6.36$ & 13.7 & -121.6 & 0.06 & -6.00 & HVC-B & \\
\hline & & 40.5 & -79.2 & 0.95 & -5.17 & $\ldots$ & $\ldots$ & $\cdots$ & $<-5.91$ & 38.3 & -77.4 & 0.47 & -5.11 & HVC-B & \\
\hline & & 30.4 & -11.1 & 0.27 & -5.72 & 22.8 & -12.3 & 0.02 & -6.45 & 26.9 & -12.2 & 0.34 & -5.26 & LVC-NC & \\
\hline \multirow[t]{2}{*}{5} & V409 Tau & 113.3 & -85.4 & 0.92 & -5.58 & $\ldots$ & $\ldots$ & $\ldots$ & $<-5.81$ & 107.6 & -64.8 & 0.22 & -5.50 & HVC-B & \\
\hline & & 70.8 & 42.5 & 0.29 & -6.08 & $\cdots$ & $\cdots$ & $\ldots$ & $<-5.95$ & 79.3 & 51.5 & 0.10 & -5.83 & HVC-R & \\
\hline \multirow[t]{2}{*}{6} & BP Tau & 94.7 & 11.3 & 0.36 & -5.22 & 88.3 & 4.0 & 0.17 & -5.24 & 98.9 & 12.4 & 0.26 & -5.04 & LVC-BC & \\
\hline & & 37.5 & -18.2 & 0.11 & -5.73 & 27.5 & -6.1 & 0.03 & -6.01 & 26.3 & -2.6 & 0.10 & -5.44 & LVC-NC & \\
\hline \multirow[t]{3}{*}{7} & DK Tau A & 45.1 & -125.6 & 0.16 & -5.30 & $\ldots$ & $\cdots$ & $\cdots$ & $<-5.69$ & 41.0 & -135.7 & 0.09 & -5.27 & HVC-B & \\
\hline & & 124.2 & -18.1 & 0.29 & -5.05 & 96.8 & -1.4 & 0.16 & -5.02 & 153.7 & -27.4 & 0.42 & -4.59 & LVC-BC & \\
\hline & & $\ldots$ & $\ldots$ & $\ldots$ & $<-5.79$ & 14.2 & 1.0 & 0.02 & -5.88 & 24.9 & -5.1 & 0.13 & -5.09 & LVC-NC & \\
\hline \multirow[t]{2}{*}{8} & HN Tau A & 116.2 & -78.5 & 2.80 & -4.25 & 183.1 & -57.9 & 0.23 & -5.19 & 132.4 & -67.7 & 2.99 & -4.07 & HVC-B & \\
\hline & & 60.2 & -9.6 & 1.44 & -4.54 & 45.3 & -0.0 & 0.10 & -5.56 & 50.4 & -6.5 & 1.33 & -4.42 & LVC-BC & HVC-B \\
\hline 9 & UX Tau A & $\ldots$ & $\ldots$ & $\ldots$ & $<-5.20$ & $\ldots$ & $\ldots$ & $\ldots$ & $<-5.18$ & 34.6 & -1.2 & 0.11 & -4.70 & LVC-NC & \\
\hline \multirow[t]{2}{*}{10} & GK Tau A & 160.6 & -22.4 & 0.36 & -4.87 & 116.6 & -35.5 & 0.04 & -5.57 & 179.1 & -30.7 & 0.38 & -4.58 & HVC-B & \\
\hline & & 36.0 & -1.0 & 0.13 & -5.31 & 51.5 & 3.1 & 0.05 & -5.48 & 38.6 & -3.3 & 0.17 & -4.94 & LVC-NC & \\
\hline \multirow[t]{3}{*}{11} & GI Tau & 86.3 & -51.9 & 0.20 & -4.79 & 38.9 & -52.6 & 0.03 & -5.73 & 85.9 & -67.5 & 0.19 & -4.85 & HVC-B & \\
\hline & & 33.6 & 46.3 & 0.15 & -4.91 & 54.1 & 40.6 & 0.05 & -5.44 & 31.7 & 46.7 & 0.17 & -4.90 & HVC-R & \\
\hline & & 39.3 & 6.2 & 0.08 & -5.20 & 59.0 & -11.5 & 0.06 & -5.38 & 44.7 & 1.3 & 0.16 & -4.92 & LVC-BC & \\
\hline 12 & DM Tau & $\ldots$ & $\ldots$ & $\ldots$ & $<-6.17$ & 24.8 & -2.0 & 0.05 & -6.51 & 26.1 & 0.5 & 0.35 & -5.69 & LVC-NC & \\
\hline 13 & LkCa 15 & $\ldots$ & $\ldots$ & $\ldots$ & $<-5.47$ & $\ldots$ & $\ldots$ & $\ldots$ & $<-5.54$ & 44.6 & -0.6 & 0.14 & -5.06 & LVC-BC & \\
\hline 14 & DS Tau & $\ldots$ & $\ldots$ & $\ldots$ & $<-4.86$ & $\ldots$ & $\ldots$ & $\ldots$ & $<-4.93$ & 151.1 & 16.3 & 0.19 & -5.05 & LVC-BC & \\
\hline 15 & SZ 65 & $\cdots$ & $\cdots$ & $\cdots$ & $<-4.92$ & $\cdots$ & $\cdots$ & $\ldots$ & $<-4.87$ & 109.9 & 3.6 & 0.25 & -4.74 & LVC-BC & \\
\hline 16 & SZ 68A & $\ldots$ & $\ldots$ & $\cdots$ & $<-6.55$ & $\ldots$ & $\ldots$ & $\ldots$ & $<-5.49$ & $\ldots$ & $\ldots$ & $\cdots$ & $<-5.50$ & $\ldots$ & $\ldots$ \\
\hline \multirow[t]{3}{*}{17} & SZ 73 & 82.5 & -88.4 & 0.70 & -4.89 & $\ldots$ & $\ldots$ & $\ldots$ & $<-5.39$ & 71.1 & -94.4 & 1.67 & -4.43 & HVC-B & \\
\hline & & $\ldots$ & $\ldots$ & $\ldots$ & $<-5.09$ & $\ldots$ & $\ldots$ & $\ldots$ & $<-5.27$ & 94.2 & 64.2 & 0.25 & -5.26 & HVC-R & \\
\hline & & $\ldots$ & $\ldots$ & $\cdots$ & $<-5.35$ & 41.1 & -11.9 & 0.11 & -5.66 & 50.8 & -19.2 & 0.69 & -4.82 & LVC-BC & \\
\hline \multirow[t]{2}{*}{18} & HM Lup & 68.3 & -119.7 & 0.50 & -5.78 & $\ldots$ & $\ldots$ & $\ldots$ & $<-5.70$ & 90.4 & -116.9 & 0.59 & -5.38 & HVC-B & \\
\hline & & $\ldots$ & $\ldots$ & $\ldots$ & $<-6.41$ & $\ldots$ & $\ldots$ & $\ldots$ & $<-6.31$ & 22.1 & -3.8 & 0.22 & -5.81 & LVC-NC & \\
\hline 19 & GW Lup & $\cdots$ & $\cdots$ & $\cdots$ & $<-5.99$ & 68.8 & -7.5 & 0.09 & -5.93 & 69.4 & -9.7 & 0.29 & -5.41 & LVC-BC & \\
\hline 20 & GQ Lup & $\cdots$ & $\ldots$ & $\cdots$ & $<-4.56$ & 46.1 & 10.7 & 0.04 & -5.04 & 91.4 & 4.0 & 0.28 & -4.18 & LVC-BC & \\
\hline 21 & SZ 76 & $\cdots$ & $\ldots$ & $\cdots$ & $<-5.80$ & 69.9 & 31.7 & 0.22 & -5.86 & 50.1 & 38.8 & 0.39 & -5.66 & HVC-R & \\
\hline & & 77.9 & -16.0 & 0.48 & -5.64 & 53.1 & -26.4 & 0.24 & -5.81 & 65.5 & -18.3 & 1.21 & -5.16 & LVC-BC & \\
\hline 22 & RU Lup & 130.0 & -137.8 & 1.68 & -3.68 & $\cdots$ & $\cdots$ & $\cdots$ & $<-4.67$ & 109.9 & -149.3 & 0.61 & -4.01 & HVC-B & \\
\hline & & 68.5 & -38.9 & 0.26 & -4.49 & $\ldots$ & $\ldots$ & $\ldots$ & $<-5.10$ & 40.5 & -32.3 & 0.07 & -4.92 & HVC-B & \\
\hline & & 150.3 & -13.1 & 0.43 & -4.28 & 120.5 & -11.7 & 0.21 & -4.47 & 176.4 & -10.1 & 0.41 & -4.18 & LVC-BC & \\
\hline & & $\ldots$ & $\ldots$ & $\cdots$ & $<-5.21$ & $\ldots$ & $\ldots$ & $\ldots$ & $<-5.57$ & 13.9 & -10.9 & 0.06 & -5.02 & LVC-NC & \\
\hline 23 & IM Lup & $\ldots$ & $\ldots$ & $\ldots$ & $<-4.98$ & $\ldots$ & $\ldots$ & $\ldots$ & $<-4.83$ & 117.1 & -10.0 & 0.16 & -4.79 & LVC-BC & \\
\hline 24 & RY Lup & $\ldots$ & $\ldots$ & $\ldots$ & $<-5.18$ & $\ldots$ & $\ldots$ & $\ldots$ & $<-5.13$ & 30.7 & 2.5 & 0.09 & -4.64 & LVC-NC & \\
\hline 25 & SZ 102 & 206.1 & -32.5 & 20.69 & -4.54 & $\ldots$ & $\ldots$ & $\cdots$ & $<-5.54$ & 209.3 & -38.1 & 22.13 & -4.63 & HVC-B & \\
\hline & & 58.3 & -25.8 & 17.62 & -4.61 & 30.7 & -16.0 & 0.89 & -5.92 & 40.0 & -24.5 & 17.99 & -4.72 & LVC-NC & HVC-B \\
\hline
\end{tabular}


Table 2

(Continued)

\begin{tabular}{|c|c|c|c|c|c|c|c|c|c|c|c|c|c|c|c|}
\hline \multirow[b]{2}{*}{ ID } & \multirow[b]{2}{*}{ Name } & \multicolumn{4}{|c|}{ [S II] $\lambda 4068$} & \multicolumn{4}{|c|}{ [O I] $\lambda 5577$} & \multicolumn{4}{|c|}{ [O I] $\lambda 6300$} & \multirow[b]{2}{*}{$\begin{array}{l}\text { Preliminary } \\
\text { Class }\end{array}$} & \multirow[b]{2}{*}{$\begin{array}{c}\text { Refined } \\
\text { Class }\end{array}$} \\
\hline & & $\begin{array}{c}\text { FWHM } \\
\left(\mathrm{km} \mathrm{s}^{-1}\right)\end{array}$ & $\begin{array}{c}v_{\mathrm{c}} \\
\left(\mathrm{km} \mathrm{s}^{-1}\right)\end{array}$ & $\begin{array}{l}\text { EW } \\
(\AA)\end{array}$ & $\begin{array}{c}\log L_{4069} \\
\left(L_{\odot}\right)\end{array}$ & $\begin{array}{c}\text { FWHM } \\
\left(\mathrm{km} \mathrm{s}^{-1}\right)\end{array}$ & $\begin{array}{c}v_{\mathrm{c}} \\
\left(\mathrm{km} \mathrm{s}^{-1}\right)\end{array}$ & $\begin{array}{l}\mathrm{EW} \\
(\AA)\end{array}$ & $\begin{array}{c}\log L_{5577} \\
\left(L_{\odot}\right)\end{array}$ & $\begin{array}{c}\text { FWHM } \\
\left(\mathrm{km} \mathrm{s}^{-1}\right)\end{array}$ & $\begin{array}{c}v_{\mathrm{c}} \\
\left(\mathrm{km} \mathrm{s}^{-1}\right)\end{array}$ & $\begin{array}{l}\mathrm{EW} \\
(\AA)\end{array}$ & $\begin{array}{c}\log L_{6300} \\
\left(L_{\odot}\right)\end{array}$ & & \\
\hline & & 85.3 & 20.6 & 53.70 & -4.13 & 108.9 & 5.9 & 4.45 & -5.22 & 93.6 & 11.8 & 127.33 & -3.87 & LVC-BC & HVC-R \\
\hline 26 & SZ 111 & $\ldots$ & $\ldots$ & $\ldots$ & $<-6.16$ & $\ldots$ & $\ldots$ & $\ldots$ & $<-5.91$ & 28.0 & -0.4 & 0.41 & -5.24 & LVC-NC & \\
\hline \multirow[t]{2}{*}{27} & SZ 98 & 167.4 & -50.9 & 2.05 & -4.46 & 108.9 & -12.4 & 0.21 & -5.13 & 152.5 & -37.7 & 1.07 & -4.40 & HVC-B & \\
\hline & & 30.4 & -8.1 & 0.21 & -5.46 & $\ldots$ & $\ldots$ & $\ldots$ & $<-5.75$ & 28.7 & -6.7 & 0.12 & -5.35 & LVC-NC & \\
\hline \multirow[t]{6}{*}{28} & EX Lup & $\ldots$ & $\ldots$ & $\ldots$ & $\ldots$ & $\ldots$ & $\ldots$ & $\ldots$ & $<-4.98$ & 25.1 & -119.3 & 0.08 & -4.53 & HVC-B & \\
\hline & & 84.3 & -181.2 & 0.17 & -3.86 & $\ldots$ & $\ldots$ & $\ldots$ & $<-4.48$ & 79.6 & -179.2 & 0.26 & -4.03 & HVC-B ${ }^{a}$ & \\
\hline & & 121.9 & -88.0 & 0.81 & -3.19 & $\ldots$ & $\ldots$ & $\ldots$ & $<-4.69$ & 48.9 & -79.5 & 0.14 & -4.29 & HVC-B & \\
\hline & & $\ldots$ & $\ldots$ & $\ldots$ & $\ldots$ & $\ldots$ & $\ldots$ & $\ldots$ & $<-4.88$ & 31.5 & 102.8 & 0.06 & -4.64 & HVC-R & \\
\hline & & $\ldots$ & $\ldots$ & $\ldots$ & $\ldots$ & $\ldots$ & $\ldots$ & $\ldots$ & $<-4.88$ & 31.1 & 56.9 & 0.11 & -4.41 & HVC-R & \\
\hline & & 37.8 & -15.0 & 0.10 & -4.11 & 65.1 & -15.8 & 0.07 & -4.53 & 59.6 & -17.6 & 0.45 & -3.79 & LVC-BC & \\
\hline \multirow[t]{4}{*}{29} & As $205 \mathrm{~A}$ & $\ldots$ & $\ldots$ & $\ldots$ & $\ldots$ & $\ldots$ & $\ldots$ & $\ldots$ & $<-5.09$ & 56.3 & -221.9 & 0.11 & -4.69 & HVC-B & \\
\hline & & 154.2 & -112.1 & 0.29 & -4.29 & $\ldots$ & $\ldots$ & $\ldots$ & $<-4.49$ & 225.5 & -85.4 & 0.28 & -4.27 & HVC-B ${ }^{a}$ & \\
\hline & & 60.4 & -19.0 & 0.12 & -4.67 & 50.8 & -0.7 & 0.03 & -5.18 & 54.5 & -13.5 & 0.16 & -4.50 & LVC-BC & \\
\hline & & 18.9 & 1.5 & 0.03 & -5.36 & 12.7 & 1.1 & 0.02 & -5.40 & 14.5 & -0.7 & 0.11 & -4.66 & LVC-NC & \\
\hline 30 & DoAr 21 & $\ldots$ & $\ldots$ & $\ldots$ & $\ldots$ & $\ldots$ & $\cdots$ & $\ldots$ & $<-4.31$ & $\ldots$ & $\cdots$ & $\cdots$ & $<-4.51$ & $\ldots$ & \\
\hline 31 & DoAr 24E & $\ldots$ & $\ldots$ & $\ldots$ & $\ldots$ & $\ldots$ & $\ldots$ & $\ldots$ & $<-5.69$ & $\ldots$ & $\ldots$ & $\ldots$ & $<-5.62$ & $\ldots$ & \\
\hline 32 & DoAr 44 & $\ldots$ & $\ldots$ & $\ldots$ & $<-5.04$ & 35.0 & 3.8 & 0.02 & -5.68 & 59.6 & -1.1 & 0.15 & -4.82 & LVC-BC & \\
\hline 33 & SR 21A & $\ldots$ & $\ldots$ & $\ldots$ & $<-3.29$ & $\ldots$ & $\ldots$ & $\ldots$ & $<-4.45$ & 16.3 & -6.9 & 0.01 & -4.81 & LVC-NC & \\
\hline \multirow[t]{3}{*}{34} & V853 Oph & 30.0 & -33.8 & 1.26 & -5.27 & $\ldots$ & $\ldots$ & $\ldots$ & $<-6.10$ & 28.5 & -31.3 & 0.93 & -5.00 & HVC-B & \\
\hline & & 68.4 & -16.0 & 1.19 & -5.30 & 37.5 & -12.4 & 0.24 & -5.62 & 70.2 & -14.4 & 1.31 & -4.85 & LVC-BC & \\
\hline & & $\ldots$ & $\ldots$ & $\ldots$ & $<-6.52$ & $\ldots$ & $\ldots$ & $\ldots$ & $<-6.33$ & 16.6 & -1.6 & 0.31 & -5.48 & LVC-NC & \\
\hline 35 & RNO 90 & $\ldots$ & $\ldots$ & $\ldots$ & $<-4.14$ & 56.1 & -3.0 & 0.06 & -4.73 & 57.5 & -3.4 & 0.23 & -4.17 & LVC-BC & \\
\hline 36 & V2508 Oph & $\ldots$ & $\ldots$ & $\ldots$ & $<-4.49$ & $\ldots$ & $\ldots$ & $\ldots$ & $<-4.67$ & 116.2 & -38.4 & 0.11 & -4.85 & HVC-B & \\
\hline 37 & V1121 Oph & $\ldots$ & $\ldots$ & $\ldots$ & $<-5.64$ & $\ldots$ & $\ldots$ & $\ldots$ & $<-5.54$ & 21.1 & -7.2 & 0.03 & -5.48 & LVC-NC & \\
\hline \multirow[t]{3}{*}{38} & $\mathrm{~J} 1842$ & 36.5 & -122.1 & 0.06 & -5.60 & $\ldots$ & $\ldots$ & $\ldots$ & $<-5.43$ & 45.6 & -126.3 & 0.10 & -5.18 & HVC-B & \\
\hline & & 83.9 & -16.4 & 0.28 & -4.94 & 93.0 & -3.1 & 0.08 & -5.29 & 109.2 & -11.5 & 0.35 & -4.64 & LVC-BC & \\
\hline & & 25.9 & 1.8 & 0.08 & -5.50 & 23.4 & -1.0 & 0.03 & -5.71 & 27.3 & -0.5 & 0.23 & -4.82 & LVC-NC & \\
\hline 39 & J1852 & $\ldots$ & $\ldots$ & $\ldots$ & $<-5.86$ & $\ldots$ & $\ldots$ & $\ldots$ & $<-5.88$ & 23.5 & -2.4 & 0.09 & -5.35 & LVC-NC & \\
\hline \multirow[t]{5}{*}{40} & VV CrA & $\ldots$ & $\ldots$ & $\ldots$ & $<-3.60$ & 85.2 & -416.1 & 0.06 & -5.06 & 133.2 & -361.6 & 0.78 & -3.88 & HVC-B & \\
\hline & & 135.5 & -209.3 & 1.88 & -3.52 & 94.7 & -234.8 & 0.30 & -4.37 & 119.7 & -205.4 & 1.58 & -3.57 & HVC-B ${ }^{a}$ & \\
\hline & & 147.8 & -88.8 & 3.89 & -3.21 & 101.5 & -78.5 & 0.14 & -4.71 & 114.2 & -87.0 & 1.73 & -3.53 & HVC-B & \\
\hline & & 35.7 & -22.5 & 0.48 & -4.11 & 51.4 & -19.8 & 0.18 & -4.59 & 42.2 & -22.1 & 0.69 & -3.93 & LVC-BC & \\
\hline & & $\ldots$ & $\ldots$ & $\ldots$ & $<-4.34$ & $\ldots$ & $\ldots$ & $\ldots$ & $<-4.99$ & 18.1 & -6.7 & 0.29 & -4.30 & LVC-NC & \\
\hline \multirow[t]{3}{*}{41} & $\mathrm{SCrA} \mathrm{A}+\mathrm{B}$ & 44.1 & -129.3 & 0.24 & -4.67 & $\ldots$ & $\ldots$ & $\ldots$ & $<-5.02$ & 45.6 & -127.7 & 0.51 & -3.91 & HVC-B & \\
\hline & & 132.7 & -72.3 & 0.60 & -4.27 & 121.1 & -40.4 & 0.09 & -4.65 & 128.0 & -49.5 & 0.66 & -3.80 & HVC-B & \\
\hline & & 58.5 & 59.1 & 0.07 & -5.19 & $\ldots$ & $\ldots$ & $\ldots$ & $<-4.85$ & 68.2 & 68.0 & 0.08 & -4.70 & HVC-R & \\
\hline 42 & TW Hya & 15.9 & -0.3 & 0.08 & -6.29 & 9.6 & 0.1 & 0.08 & -5.89 & 12.8 & 0.0 & 0.57 & -5.03 & LVC-NC & \\
\hline 43 & TWA 3A & $\ldots$ & $\ldots$ & $\ldots$ & $<-7.03$ & 56.0 & -1.1 & 0.11 & -6.98 & 61.9 & 1.3 & 0.59 & -6.09 & LVC-BC & \\
\hline \multirow[t]{3}{*}{44} & V1057 Cyg & $\ldots$ & $\ldots$ & $\ldots$ & $<-1.61$ & $\ldots$ & $\ldots$ & $\ldots$ & $<-2.51$ & 50.0 & -154.6 & 0.23 & -1.88 & HVC-B & \\
\hline & & $\ldots$ & $\ldots$ & $\ldots$ & $<-1.55$ & $\ldots$ & $\ldots$ & $\ldots$ & $<-2.45$ & 58.0 & -98.6 & 0.24 & -1.86 & HVC-B & \\
\hline & & $\ldots$ & $\ldots$ & $\ldots$ & $<-1.27$ & $\ldots$ & $\ldots$ & $\ldots$ & $<-2.17$ & 110.9 & -60.0 & 0.25 & -1.85 & HVC-B & \\
\hline \multirow[t]{2}{*}{45} & V1515 Cyg & $\ldots$ & $\ldots$ & $\ldots$ & $<-3.05$ & $\ldots$ & $\ldots$ & $\ldots$ & $<-3.28$ & 32.1 & -120.8 & 0.03 & -3.45 & HVC-B & \\
\hline & & $\ldots$ & $\ldots$ & $\ldots$ & $<-2.72$ & $\ldots$ & $\ldots$ & $\ldots$ & $<-2.94$ & 69.9 & -63.2 & 0.18 & -2.73 & HVC-B & \\
\hline \multirow[t]{2}{*}{46} & HD 143006 & $\ldots$ & $\ldots$ & $\ldots$ & $<-4.37$ & $\ldots$ & $\ldots$ & $\cdots$ & $<-4.73$ & 66.3 & 2.8 & 0.09 & -4.45 & LVC-BC & \\
\hline & & $\ldots$ & $\ldots$ & $\ldots$ & $<-4.91$ & $\ldots$ & $\ldots$ & $\ldots$ & $<-5.27$ & 19.1 & 2.1 & 0.02 & -5.02 & LVC-NC & \\
\hline
\end{tabular}


Table 2

(Continued)

\begin{tabular}{|c|c|c|c|c|c|c|c|c|c|c|c|c|c|c|c|}
\hline \multirow[b]{2}{*}{ ID } & \multirow[b]{2}{*}{ Name } & \multicolumn{4}{|c|}{ [S II] $\lambda 4068$} & \multicolumn{4}{|c|}{ [O I] $\lambda 5577$} & \multicolumn{4}{|c|}{$[\mathrm{O}$ I] $\lambda 6300$} & \multirow[b]{2}{*}{$\begin{array}{l}\text { Preliminary } \\
\text { Class }\end{array}$} & \multirow[b]{2}{*}{$\begin{array}{l}\text { Refined } \\
\text { Class }\end{array}$} \\
\hline & & $\begin{array}{l}\text { FWHM } \\
\left(\mathrm{km} \mathrm{s}^{-1}\right)\end{array}$ & $\begin{array}{c}v_{\mathrm{c}} \\
\left(\mathrm{km} \mathrm{s}^{-1}\right)\end{array}$ & $\begin{array}{l}\text { EW } \\
(\AA)\end{array}$ & $\begin{array}{c}\log L_{4069} \\
\left(L_{\odot}\right)\end{array}$ & $\begin{array}{c}\text { FWHM } \\
\left(\mathrm{km} \mathrm{s}^{-1}\right)\end{array}$ & $\begin{array}{c}v_{\mathrm{c}} \\
\left(\mathrm{km} \mathrm{s}^{-1}\right)\end{array}$ & $\begin{array}{l}\text { EW } \\
(\AA)\end{array}$ & $\begin{array}{c}\log L_{5577} \\
\quad\left(L_{\odot}\right)\end{array}$ & $\begin{array}{c}\text { FWHM } \\
\left(\mathrm{km} \mathrm{s}^{-1}\right)\end{array}$ & $\begin{array}{c}v_{\mathrm{c}} \\
\left(\mathrm{km} \mathrm{s}^{-1}\right)\end{array}$ & $\begin{array}{l}\mathrm{EW} \\
(\AA)\end{array}$ & $\begin{array}{c}\log L_{6300} \\
\left(L_{\odot}\right)\end{array}$ & & \\
\hline 47 & DI Cep & $\ldots$ & $\ldots$ & $\ldots$ & $<-4.75$ & $\ldots$ & $\cdots$ & $\ldots$ & $<-4.69$ & 27.3 & -7.5 & 0.11 & -4.18 & LVC-NC & \\
\hline \multirow[t]{4}{*}{48} & As $353 \mathrm{~A}$ & $\ldots$ & $\ldots$ & $\ldots$ & $\ldots$ & $\ldots$ & $\ldots$ & $\ldots$ & $<-4.77$ & 48.4 & -280.0 & 0.21 & -4.25 & HVC-B & \\
\hline & & 318.0 & -128.6 & 1.96 & -3.67 & $\ldots$ & $\ldots$ & $\ldots$ & $<-3.96$ & 322.8 & -126.6 & 0.94 & -3.61 & HVC-B ${ }^{\mathrm{a}}$ & \\
\hline & & 95.1 & -43.2 & 0.41 & -4.35 & $\ldots$ & $\ldots$ & $\ldots$ & $<-4.66$ & 62.7 & -27.9 & 0.13 & -4.47 & LVC-BC & Suspicious \\
\hline & & $\ldots$ & $\ldots$ & $\ldots$ & $\ldots$ & $\ldots$ & $\ldots$ & $\ldots$ & $<-4.99$ & 29.1 & -4.2 & 0.09 & -4.62 & LVC-NC & \\
\hline
\end{tabular}

Note.

The fitted parameters for the components from [S II] $\lambda 4068$ are unreliable. 


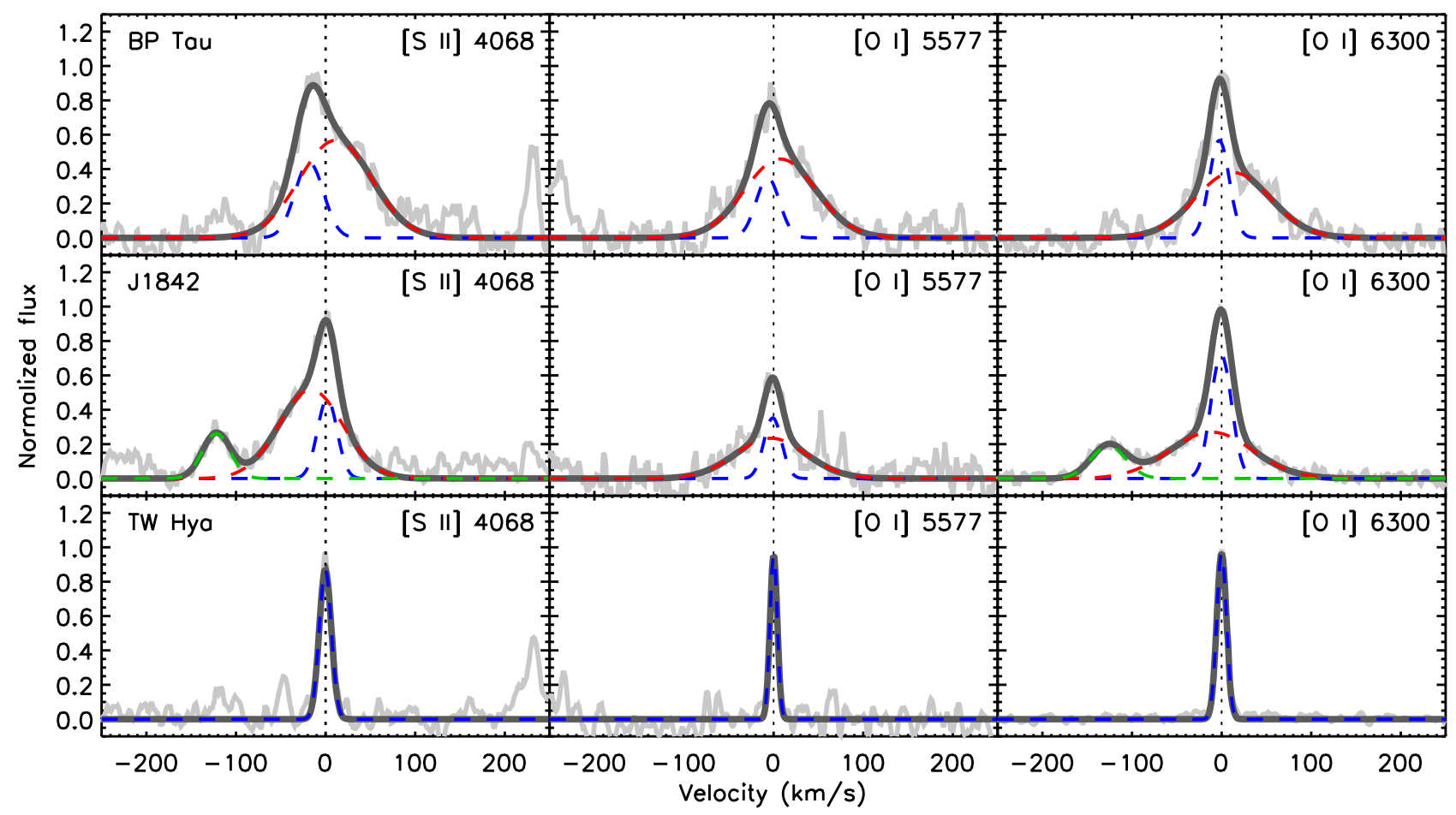

Figure 3. Examples of [S II] $\lambda 4068$, [O I] $\lambda 6300$, and [O I] $\lambda 5577$ line profile fits. A green dashed line is used for the HVC, a red dashed line for the LVC-BC, and a blue dashed line for the LVC-NC. The dark solid line is the sum of the individual components. Line profile fits for all sources are shown in Figure 19.

LVC-NC. Finally, only $20 \%(9 / 45)$ of our sources have both LVC-BC and LVC-NC components.

\section{Thermal [O I] Emission and Associated Gas Properties}

\subsection{The Similarity of [S II] and [O I] Profiles of Individual Kinematic Components}

Figure 5 shows a comparison of HVC, LVC-NC, and LVC$\mathrm{BC}$ profiles for the sources that are detected in both [S II] $\lambda 4068$ and [O I] $\lambda 6300$. Whenever detected, [O I] $\lambda 5577$ lines are also superimposed. To test how similar are the profiles of individual kinematic components, we remove from the observed profile the other components using our best-fit parameters reported in Table 2.

Regardless of kinematic component (HVC, LVC-BC, or LVC-NC), most profiles are similar in the [O I] and [S II] lines. Only BP Tau has an LVC-NC that is clearly more blueshifted in the [S II] $\lambda 4068$ than in the [O I] lines, although the line widths are actually similar. This similarity is strengthened when comparing the centroids and FWHMs of individual components (see Figures 21 and 22 in Appendix F). Thus, we conclude that, within a kinematic component, the three lines trace a similar region. This means that their line ratios can be used to constrain the properties of the emitting gas, in particular temperature and density.

\subsection{Gas Properties Constrained by Line Ratios}

The difference in the HVC and LVC forbidden-line ratios was already noted in Section 3.4 and used to improve on our preliminary kinematic classification. Here, we search for and quantify any difference in line ratios between LVC-NC, LVC$\mathrm{BC}$, and HVC. Table 3 lists the mean and standard deviation of $\log$ OI55/63 and $\log$ SII40/OI63 for three types of components when considering only detections. LVC-BCs have the largest mean log OI55/63 ratio, while HVCs have the lowest one. HVCs have the highest mean log SII40/OI63 ratio, while LVC-NCs the lowest one.

Next, we include upper limits and use Gehan's generalized Wilcoxon test in the ASURV code (Feigelson \& Nelson 1985) to quantify any statistically significant difference between pairs of kinematic components. The test finds a low probability that the HVC $\log$ OI55/63 and $\log$ SII40/OI63 are the same as those of the LVC-BC. HVC and LVC-NC also have statistically different $\log \mathrm{SII} 40 / \mathrm{OI} 63$, but indistinguishable $\log$ OI55/63. Finally, the LVC-BC and LVC-NC differ in both $\log$ OI55/63 and $\log$ SII40/OI63; see Table 4. These findings suggest that the combination of these three forbidden lines is sufficient to separate the kinematic components discussed in this paper.

\subsubsection{Thermally Excited Gas Explains Most LVC Line Ratios}

Figure 6(a) shows the relation between the SII40/OI63 and the OI55/63 ratios for the LVC components. In the same figure we also plot predicted ratios for homogeneous and isothermal gas that is thermally excited by collisions with electrons (gray lines). When computing these ratios, we take the solar sulfur abundance, $\alpha(\mathbf{S})=1.4 \times 10^{-5}$ (Asplund et al. 2005), and a depleted oxygen abundance as in the interstellar medium, $\alpha(\mathrm{O})=3.2 \times 10^{-4}$ (Savage \& Sembach 1996). Following Natta et al. (2014), we also assume that all oxygen is neutral while all sulfur is singly ionized. Gas at higher temperature and electron density is predicted to have higher SII40/OI63 and OI55/63 line ratios.

Most of the observed LVC ratios correspond to thermally excited gas with temperatures between 5000 and $10,000 \mathrm{~K}$ and electron densities of $\sim 10^{7}-10^{8} \mathrm{~cm}^{-3}$. In As 353A, its LVC-BC has an SII40/OI63 ratio higher than expected from this simple model, with a value seen in some of the HVC. As discussed in 

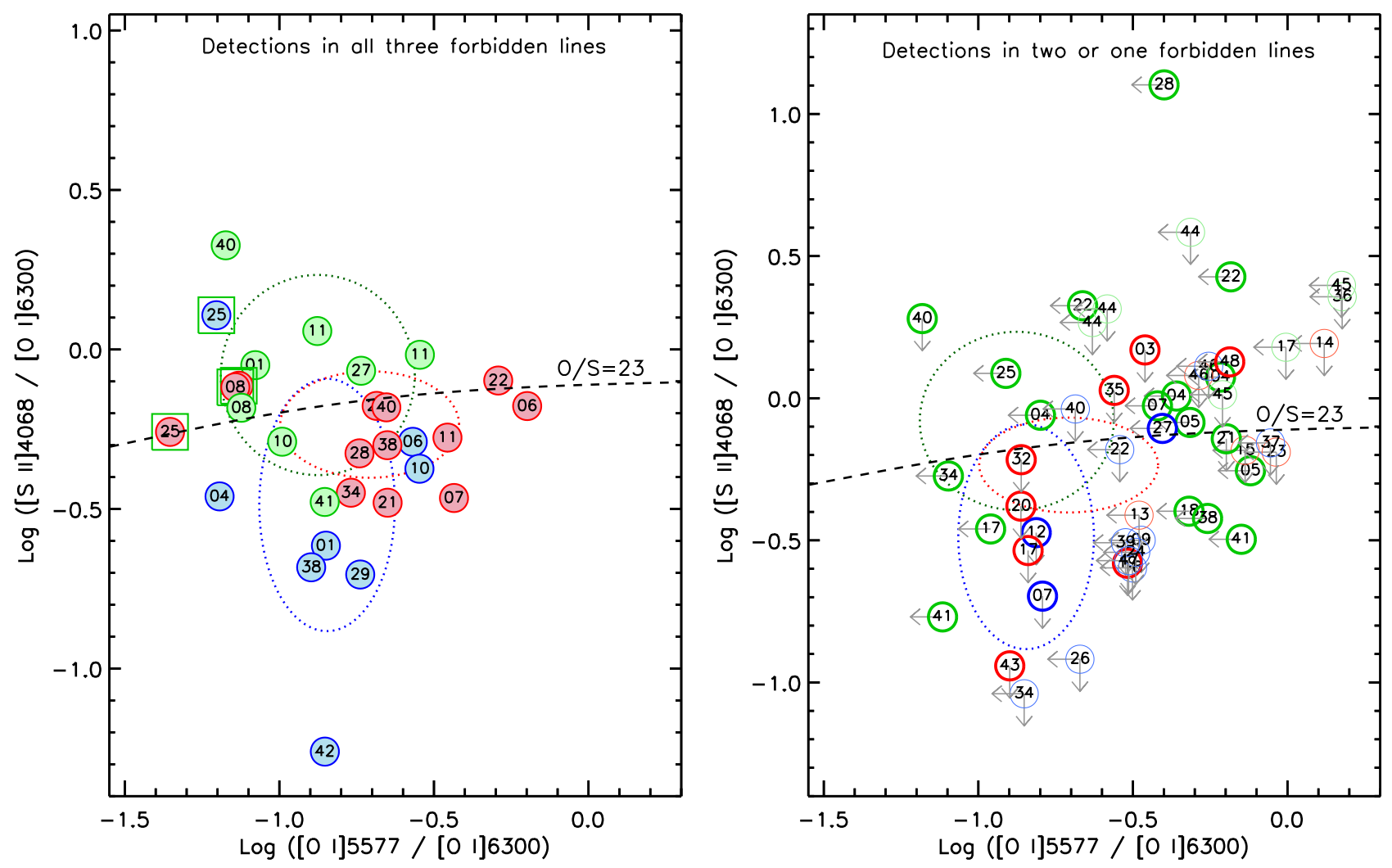

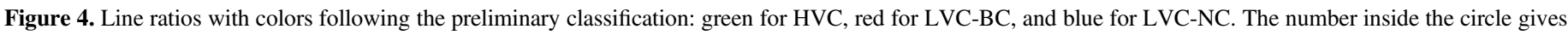

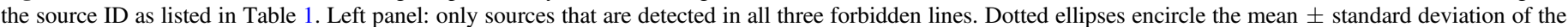

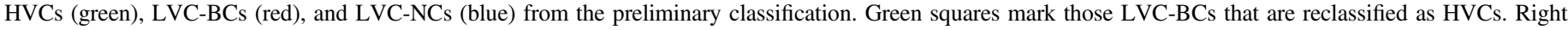

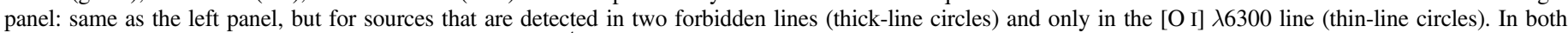

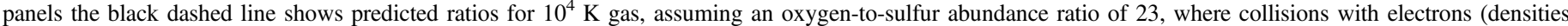
$10^{5.8}-10^{8.5} \mathrm{~cm}^{-3}$ ) excite the upper levels of the transitions; see Section 4.2.1 for more details.

Section 3.4, the [S II] $\lambda 4068$ line in this very high accretion rate star is blended with Fe I emission and classified as suspicious in Table 2, after our attempt at deblending. Turning to the LVC-NC, three of the transition disk sources, TW Hya, TWA 3A, and SZ 111, have much lower SII40/OI63 ratios than those expected for gas at $\sim 5000 \mathrm{~K}$. Gehan's generalized Wilcoxon test finds a low probability $\left(p=6 \times 10^{-2}\right)$ that the SII40/OI63 ratios of LVC-BCs of full disks and TDs are drawn from the same parent population, while their OI53/63 ratios are indistinguishable ( $p=0.3$ for both the LVC-NC and the LVC$\mathrm{BC})$. This can be explained if TDs have a decreased S II/O I abundance ratio, as could happen if sulfur is sequestered in large grains in the disk midplane.

\subsubsection{Shock-heated Gas Explains Most HVC Line Ratios}

Jets generate shock waves in the surrounding medium, where part of their kinetic energy is converted into thermal motion, thereby producing bright forbidden-line emission (e.g., Giannini et al. 2015). To further investigate the origin of the HVCs detected in our sample, we compare their [S II] and [O I] line ratios to those expected from the shock models of Hartigan \& Wright (2015); see Figure 6(b). The model line ratios were calculated for a range of input parameters: $\log n_{0}\left(\mathrm{~cm}^{-3}\right)=2-11$ with an increment of unity in $\log$ scale, $X_{e}=0.1$ and $0.6, V_{\mathrm{s}}=30-80 \mathrm{~km} \mathrm{~s}^{-1}$, $M_{\mathrm{A}}=1.5,4.6$, and 10 . Here, $n_{0}$ is the pre-shock number density of nucleons, $X_{e}$ is the pre-shock ionization fraction, $V_{\mathrm{S}}$ is the shock velocity, and $M_{\mathrm{A}}$ is the Alfvénic Mach number. We have also scaled the ratios to the atomic abundances used in
Section $4.2 .1, \alpha(\mathrm{O})=3.2 \times 10^{-4}$ and $\alpha(\mathrm{S})=1.4 \times 10^{-5}$, from the original solar abundances in Hartigan \& Wright (2015).

Shock models with a pre-shock number density of $\log n_{0}\left(\mathrm{~cm}^{-3}\right) \sim 6-7$ can reproduce most of the observed SII40/OI63 and OI55/63 HVC line ratios. As forbidden-line emission occurs in the post-shock cooling zone, the specific transition critical density and excitation temperature dictate how close to the front the emitting material is located. For instance, the [O I] $\lambda 6300$ line probes gas slightly closer to the front than the [S II] $\lambda 6731$ line (see, e.g., Figure 3 in Hartigan $\&$ Wright 2015), which has a similar excitation temperature but a critical density about 100 times lower. The [S II] $\lambda 4068$ line investigated in this work has similar temperature and density to the [O I] $\lambda 6300$ line and hence should also trace denser/hotter gas closer to the front than the $[\mathrm{S} \mathrm{II}] \lambda 6731$ line.

\section{Mass Outflow to Mass Accretion Rate}

\subsection{Mass Accretion Rates}

After flux-calibrating our spectra (see Appendix B), we estimate the accretion luminosity using 12 emission lines covered by the HIRES spectra, including five Balmer lines $(\mathrm{H} \zeta, \mathrm{H} \delta, \mathrm{H} \gamma$, $\mathrm{H} \beta$, and $\mathrm{H} \alpha$ ), four He I lines (4026, 4471, 5876, and $6678 \AA$ ), the He II line at $4686 \AA$, and the Ca II lines at 3934 and $3968 \AA$. These lines are chosen because their luminosities are known to correlate tightly with accretion luminosities (e.g., Herczeg \& Hillenbrand 2008; Fang et al. 2009; Rigliaco et al. 2012; Alcalá et al. 2014, 2017). After computing line luminosities, we convert 

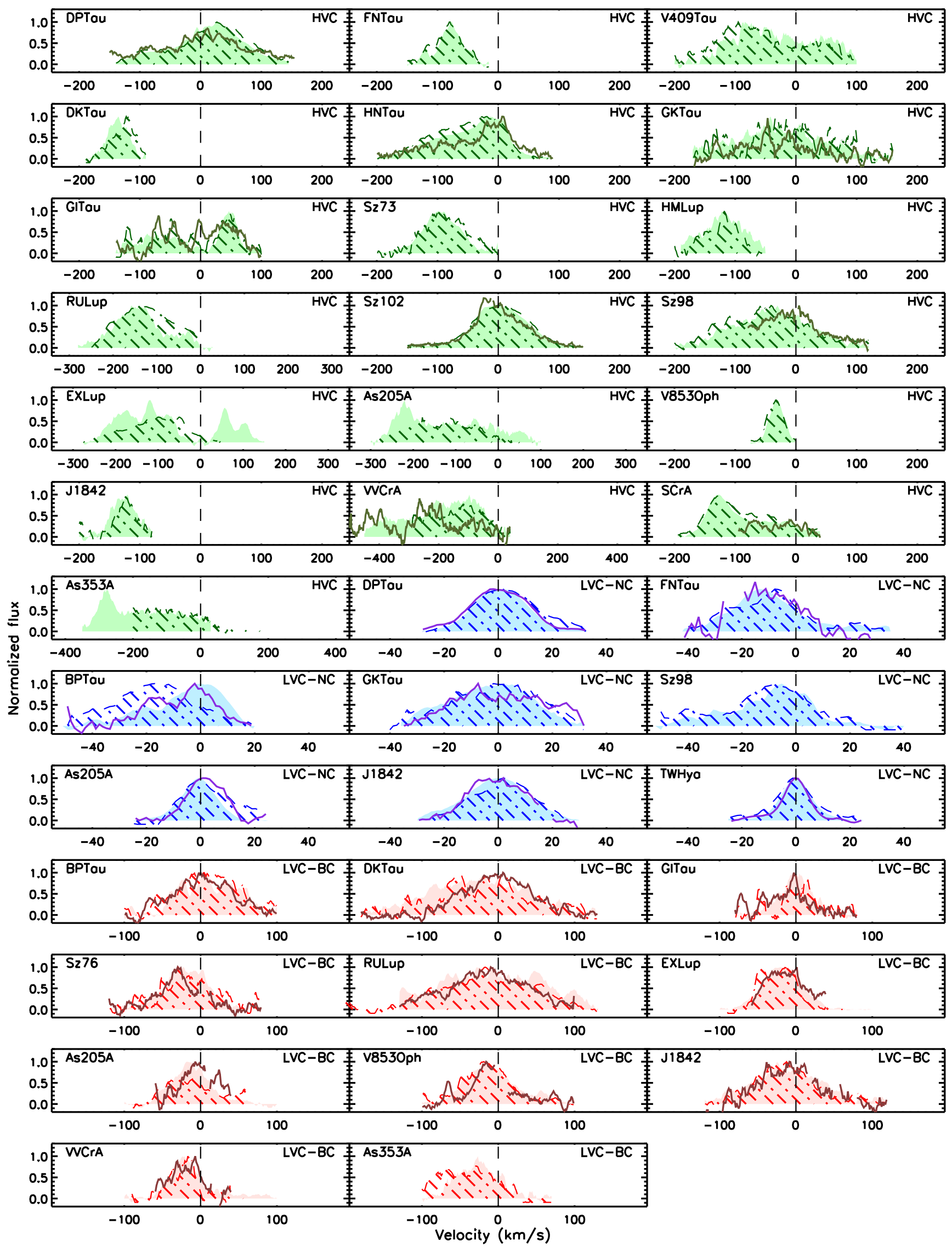

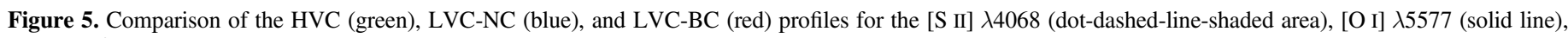
and $[\mathrm{O}$ I] $\lambda 6300$ (color-filled area). Most individual kinematic components have similar profiles in the three different forbidden lines investigated here. 
Table 3

Mean Line Ratios and Standard Deviations after HVC Reclassification

\begin{tabular}{lccc}
\hline \hline Line Ratios & LVC-NC & LVC-BC & HVC \\
\hline $\log$ OI55/63 & $-0.81 \pm 0.19$ & $-0.62 \pm 0.20$ & $-0.97 \pm 0.31$ \\
$\log$ SII40/OI63 & $-0.56 \pm 0.35$ & $-0.25 \pm 0.18$ & $-0.08 \pm 0.36$
\end{tabular}

Table 4

Results fro Gehan's Generalized Wilcoxon Test

\begin{tabular}{lcccc}
\hline \hline \multicolumn{2}{c}{$\log$ OI55/63 } & & \multicolumn{2}{c}{$\log$ SII40/OI63 } \\
\hline Pairs & Probability & & Paris & Probability \\
\hline LVC-NC/ & $p=6 \times 10^{-3}$ & & LVC-NC/ & $p=1 \times 10^{-2}$ \\
LVC-BC & & & LVC-BC & \\
LVC-NC/HVC & $p=1 \times 10^{-1}$ & & LVC-NC/HVC & $p<5 \times 10^{-5}$ \\
LVC-BC/HVC & $p<5 \times 10^{-5}$ & & LVC-BC/HVC & $p=4 \times 10^{-4}$ \\
\hline
\end{tabular}

them into accretion luminosities via the empirical relations listed in Appendix G. Table 5 lists the accretion luminosities derived from the transitions discussed above, as well as the $3 \sigma$-clipping mean of the accretion luminosities for each source. Mean accretion luminosities are converted into mass accretion rates using the following relation:

$$
\dot{M}_{\mathrm{acc}}=\frac{L_{\mathrm{acc}} R_{\star}}{\mathrm{G} M_{\star}\left(1-\frac{R_{\star}}{R_{\mathrm{in}}}\right)},
$$

where $R_{\text {in }}$ denotes the truncation radius of the disk, which is taken to be $5 R_{\star}$ (Gullbring et al. 1998), $G$ is the gravitational constant, $M_{\star}$ is the stellar mass, and $R_{\star}$ is the stellar radius. Mass accretion rates are also listed in Table 5. Our sample covers a large range in accretion luminosities and mass accretion rates, from $\log \left(L_{\text {acc }} / L_{\odot}\right)=-3.48$ to 0.72 and from $\log \left(\dot{M}_{\mathrm{acc}} / M_{\odot} \mathrm{yr}^{-1}\right)=-10.15$ to -6.12 , estimated from the accretion-related emission lines.

We do not detect any accretion-related emission lines from DoAr $21(\lambda=4420-6300 \AA)$, DoAr 24E $(\lambda=4420-6300 \AA)$, SR 21A $(\lambda=3650-6300 \AA)$, and V1057 Cyg $(\lambda=3650-$ $6300 \AA$ ); hence, we do not list their accretion luminosities in Table 5. Note that V1057 Cyg and V1515 Cyg are well-known FU Ori objects. For this class of objects accretion luminosities are best derived from the emission of their self-luminous disks (e.g., Kenyon et al. 1998), and we use the more recent estimates from Green et al. (2006) in our paper. Interestingly, the spectra of DoAr 21 and DoAr 24E do not show any [O I] $\lambda 6300$ emission, while those of SR 21A show only a marginal detection; see Appendix $\mathrm{C}$ for more details. To clarify which sources are truly accreting, we also compare the derived accretion luminosities with typical chromospheric values (see Manara et al. 2017). Only TWA 3A has an accretion luminosity below the chromospheric emission, suggesting that chromospheric activity dominates the line emission.

Figure 7(a) compares the distribution of accretion rates for full disks (gray-filled histogram) and TDs (hatch-filled histogram). The K-S test returns a low probability $\left(P \sim 1.8 \times 10^{-5}\right.$ ) for the full disks and TDs to be drawn from the same parent population. The accretion rates of TDs are systematically lower than those of full disks with a median value of $1.6 \times 10^{-9} M_{\odot} \mathrm{yr}^{-1}$, about seven times lower than that of the full disks in our sample. A similar result is reported in Najita et al. (2015) when comparing full and transition disks in Ophiuchus and Taurus. Figure 7(b) compares the distribution of stellar masses. The K-S test gives a $55 \%$ probability that the two samples are drawn from the same stellar mass distribution; hence, the difference in accretion rates most likely relates to their different evolutionary stage.

\subsection{Correlation between [OI] and Accretion Luminosity}

Previous work has shown that the [O I] $\lambda 6300$ line luminosity correlates with stellar properties like stellar and accretion luminosity but not with X-ray luminosity (e.g., Rigliaco et al. 2013; Nisini et al. 2018). Here, we repeat the analysis by combining our sample with that of Nisini et al. (2018), as they cover a large range in stellar properties, and by implementing the new Gaia distances (Bailer-Jones et al. 2018) for all samples. Our goal is to clarify which correlations are present and provide the most up-to-date relations with accurate distances for individual sources.

Figures 8(a) and (b) show the relationship between the LVC $(\mathrm{NC}+\mathrm{BC})$ and HVC [O I] $\lambda 6300$ line luminosity versus the accretion luminosity. The Spearman's rank correlation coefficients for the LVCs and HVCs are 0.79 and 0.55 , respectively, with a probability lower than $5 \times 10^{-5}$ that the data are uncorrelated. We perform outlier-resistant two-variable linear regressions including only the detections ${ }^{14}$ and obtain the following relationships:

$$
\begin{aligned}
& \log L_{\mathrm{OI} 63, \mathrm{LVC}}=(0.60 \pm 0.03) \log L_{\mathrm{acc}}-(4.07 \pm 0.06) \\
& \log L_{\mathrm{OI} 63, \mathrm{HVC}}=(0.72 \pm 0.07) \log L_{\mathrm{acc}}-(3.90 \pm 0.11) .
\end{aligned}
$$

The slope for the $L_{\mathrm{OI} 63, \mathrm{LVC}}$ versus $L_{\mathrm{acc}}$ in this work is similar to those $(0.52 \pm 0.07,0.59 \pm 0.04)$ in Rigliaco et al. (2013) and Nisini et al. (2018), but much flatter than the one $(0.81 \pm 0.09)$ in Natta et al. (2014), which could be due to their smaller sample size. Our $L_{\mathrm{OI} 63, \mathrm{HVC}}$ versus $L_{\text {acc }}$ slope is also similar to the one $(0.75 \pm 0.08)$ in Nisini et al. (2018), but slightly flatter than the one $(\sim 0.9)$ in Rigliaco et al. (2013). The $L_{\mathrm{OI} 63, \mathrm{HVC}}$ versus $L_{\text {acc }}$ slope is steeper than the $L_{\mathrm{OI} 63, \mathrm{LVC}}$ versus $L_{\mathrm{acc}}$ slope with a $2 \sigma$ confidence, consistent with the findings in Rigliaco et al. (2013). Note that, using only our sources, we find the same slopes as those reported in Equations (2) and (3) within the (now larger) uncertainties $(0.60 \pm 0.06$ and $0.62 \pm 0.16)$.

For our sources we can further correlate the line luminosity of individual LVC components with $L_{\text {acc }}$; see Figure 9. The Pearson correlation coefficients for the LVC-NC and LVC-BC are 0.71 and 0.92 with two-sided $p$-values of $1.4 \times 10^{-4}$ and $7.6 \times 10^{-10}$, respectively. This means that their luminosities are correlated with the accretion luminosity, with the LVC-BC displaying a higher correlation than the NC and the total LVC (see Figure 8(a)). The outlier-resistant two-variable linear regression gives the following relations:

$$
\log L_{\mathrm{OI} 63, \mathrm{LVC}-\mathrm{BC}}=(0.51 \pm 0.05) \log L_{\mathrm{acc}}-(4.22 \pm 0.07)
$$

$\log L_{\mathrm{OI} 63, \mathrm{LVC}-\mathrm{NC}}=(0.49 \pm 0.10) \log L_{\mathrm{acc}}-(4.53 \pm 0.14)$.

The $L_{\mathrm{OI} 63, \mathrm{LVC}-\mathrm{BC}}$ and the $L_{\mathrm{OI} 63, \mathrm{LVC}-\mathrm{NC}}$ versus $L_{\mathrm{acc}}$ slopes are the same within the uncertainties.

\footnotetext{
${ }^{14}$ Upper limits depend on the assumed line profile, which, especially for the HVC, varies substantially from source to source. In the case of line ratios, Section 4, we could include upper limits because we required the detection of at least one line, which then set the profile for the nondetections.
} 

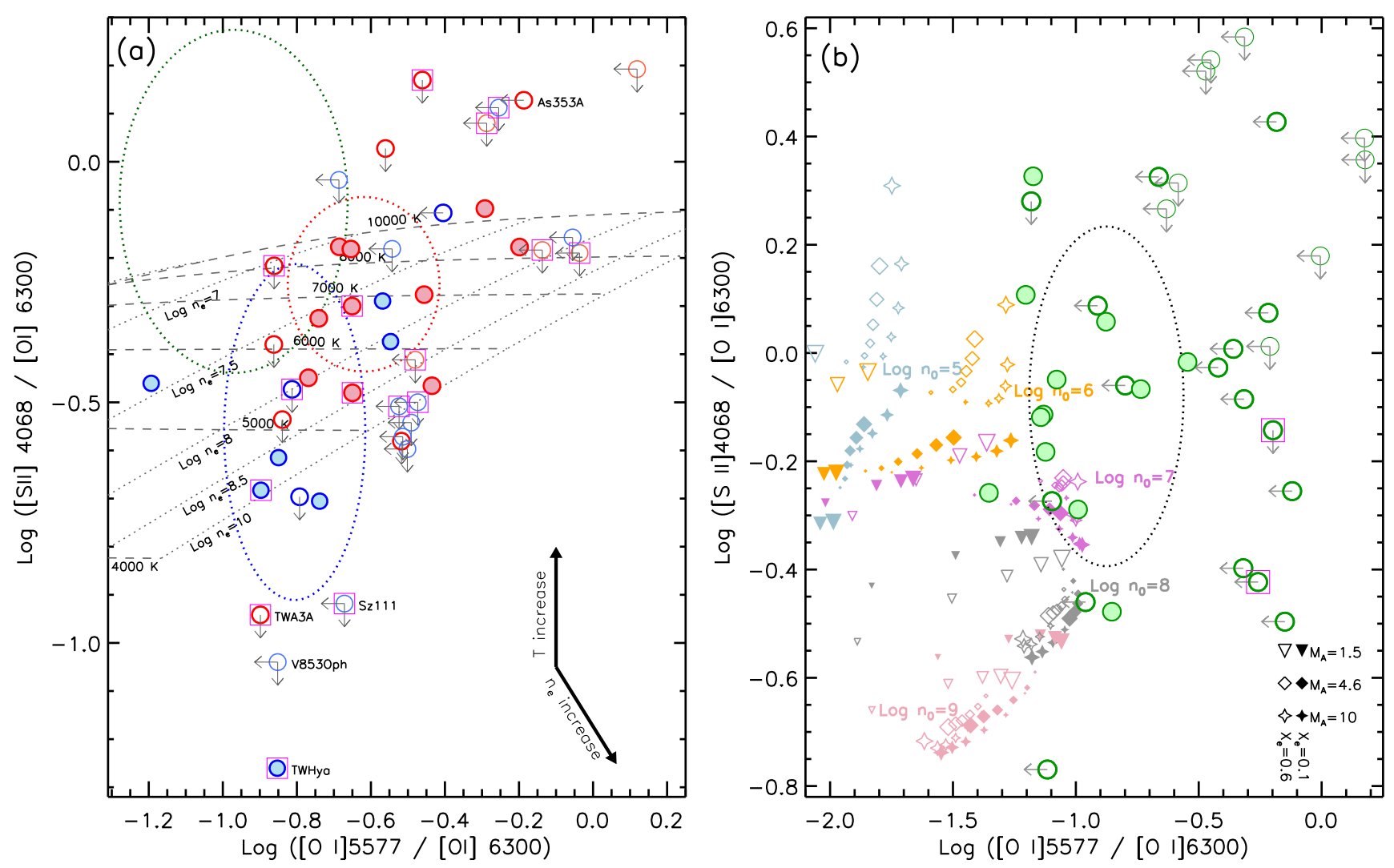

Figure 6. SII40/OI63 vs. OI55/63 line ratios for LVC-NC (blue) and LVC-BC (red) in panel (a) and HVC (green) in panel (b). In each panel, the filled circles mark sources with detections in all three forbidden lines, thick-line open circles are for those with detections in two forbidden lines, and thin-line open circles are for sources that have only an [O I] $\lambda 6300$ detection. Magenta squares mark TDs. Dotted ellipses encircle the mean \pm standard deviation of the HVCs (green), LVC-BCs (red), and LVC-NCs (blue) after the refined classification (see Section 3.4). In panel (a), observed line ratios are compared with those predicted by thermally excited gas. Gray dashed lines locate gas at temperatures from $4000 \mathrm{~K}$ (bottom) to $10,000 \mathrm{~K}$ (top), while dotted lines at different electron densities from log $n_{\mathrm{e}}\left(\mathrm{cm}^{-3}\right)=6.5$ (left) to $\log n_{\mathrm{e}}\left(\mathrm{cm}^{-3}\right)=10$ (right). In panel (b) HVC line ratios are compared with those predicted by shock models (Hartigan \& Wright 2015). Open symbols are for $X_{e}=0.6$, while filled ones are for $X_{e}=0.1$; triangles are for $M_{\mathrm{A}}=1.5$, diamonds are for $M_{\mathrm{A}}=4.6$, and stars are for a higher Mach number of 10. Different colors indicate different pre-shock number densities of nucleons with $\log n_{0}\left(\mathrm{~cm}^{-3}\right)$ from 5 to 9 . Finally, the size of the symbols scales with the shock velocity $\left(V_{\mathrm{s}}\right)$, which ranges from 30 to $80 \mathrm{~km} \mathrm{~s}^{-1}$ in these models.

\subsection{Inner Disk Evolution Traced by the [O I] Emission}

The evolutionary stage of a star+disk system is often assessed from its SED. However, optical forbidden lines can aid in understanding the evolutionary stage of a system by probing the evolution of the gas content (see, e.g., Figure 5 in Ercolano \& Pascucci 2017).

The frequency of different [O I] $\lambda 6300$ line components differs for full and TDs; see Table 6 , where we provide statistics ${ }^{15}$ for our sample. Full disks more frequently than TDs show an HVC in their [OI] $\lambda 6300$ profiles, ${ }^{16}$ as well as a BC accompanied by an NC. In contrast, TDs more frequently than full disks present only an LVC-NC or LVC-BC. In addition, TDs have simpler line profiles: $\sim 81 \%(13 / 16)$ of our TDs only show one LVC-NC or one LVC-BC without any other LVC or HVCs components, while this fraction is down to $24 \%(7 / 29)$ for the full disks $;{ }^{17}$ see also Figures 16 and 17 in Appendix A.

\footnotetext{
${ }^{15}$ Only LVC-NC means that the [O I] $\lambda 6300$ profile does not show any LVCBC component. Similarly, only LVC-BC means that there is no LVC-NC component in the $[\mathrm{O} \mathrm{I}]$ profile.

${ }^{16}$ Note that our $72 \%$ fraction of HVCs in full disks is much higher than the $\sim 30 \%$ quoted in Nisini et al. (2018), perhaps due to a bias toward strong accretors in our sample.

${ }^{17}$ We also note that among the seven full disks, showing only simple [O I] $\lambda 6300$ line profiles, two of them, DS Tau and RY Lup, could also be transition disks based on the submillimeter/millimeter data (Piétu et al. 2014; Ansdell et al. 2016).
}

With a larger sample of $65 \mathrm{~T}$ Tauri stars, Banzatti et al. (2018) also find that those surrounded by a TD tend to show only a single component in their [O I] $\lambda 6300$ line profile (see their Section 5.5 for details). The much lower fraction of TDs with an $\mathrm{HVC}$ and a $\mathrm{BC}+\mathrm{NC}$ with respect to full disks might indicate an evolution in disk winds (see also Ercolano \& Pascucci 2017). The TDs in our sample have lower mass accretion rates than full disks, and hence lower HVC and LVC luminosities (Figure 8), and likely a more depleted inner gas disk. As also suggested in Banzatti et al. (2018), winds in TDs might be launched from larger radii, have a larger opening angle, and not recollimate into jets.

Furthermore, the [O I] $\lambda 6300$ line profiles can be used to distinguish the evolutionary stage of systems with similar SEDs. An example is shown in Figure 10 for LkCa 15 and $\mathrm{J} 1842$, both classified as TDs. The central stars have similar spectral types (K5.5 vs. K3) and accretion rates $\left(1.8 \times 10^{-9}\right.$ vs. $\left.3.0 \times 10^{-9} M_{\odot} \mathrm{yr}^{-1}\right)$, and their disks present very similar SEDs. However, their forbidden-line profiles are clearly different: LkCa 15 only presents an LVC-BC (with an FWHM, $44.6 \mathrm{~km} \mathrm{~s}^{-1}$, near the boundary to separate the LVCNCs and LVC-BCs) in the [O I] $\lambda 6300$, while J1842 shows an LVC-NC, an LVC-BC, and an HVC in both the [S II] $\lambda 4068$ and [O I] $\lambda 6300$ lines and an LVC-NC and an LVC-BC in the [O I] $\lambda 5577$ line. Thus, while both sources would appear to be in the same evolutionary stage based on their SED, the 
Table 5

Accretion Luminosity and Accretion Rates of the Sources in This Work

$\log L_{\mathrm{acc}}$

\begin{tabular}{|c|c|c|c|c|c|c|c|c|c|c|c|c|c|c|c|}
\hline \multirow[b]{2}{*}{ ID } & \multirow[b]{2}{*}{ Name } & \multicolumn{12}{|c|}{$\log L_{\text {acc }}$} & \multirow[b]{2}{*}{$\begin{array}{c}\log \overline{L_{\text {acc }}} \\
\left(L_{\odot}\right)\end{array}$} & \multirow[b]{2}{*}{$\begin{array}{l}\log \dot{M}_{\text {acc }} \\
\left(M_{\odot} \mathrm{yr}^{-1}\right)\end{array}$} \\
\hline & & $\begin{array}{l}(\mathrm{H} \zeta) \\
\left(L_{\odot}\right)\end{array}$ & $\begin{array}{l}(\mathrm{H} \delta) \\
\left(L_{\odot}\right)\end{array}$ & $\begin{array}{l}(\mathrm{H} \gamma) \\
\left(L_{\odot}\right)\end{array}$ & $\begin{array}{l}(\mathrm{H} \beta) \\
\left(L_{\odot}\right)\end{array}$ & $\begin{array}{l}(\mathrm{H} \alpha) \\
\left(L_{\odot}\right)\end{array}$ & $\begin{array}{c}(\mathrm{He} \text { I } \lambda 4026) \\
\left(L_{\odot}\right)\end{array}$ & $\begin{array}{c}(\mathrm{He} \text { I } \lambda 4471) \\
\left(L_{\odot}\right)\end{array}$ & $\begin{array}{c}(\mathrm{He} \text { I } \lambda 5876) \\
\left(L_{\odot}\right)\end{array}$ & $\begin{array}{c}(\mathrm{He} \text { I } \lambda 6678) \\
\left(L_{\odot}\right)\end{array}$ & $\begin{array}{c}(\mathrm{He} \text { II } \lambda 4686) \\
\left(L_{\odot}\right)\end{array}$ & $\begin{array}{c}(\mathrm{Ca} \text { II } \lambda 3934) \\
\left(L_{\odot}\right)\end{array}$ & $\begin{array}{c}(\mathrm{Ca} \text { II } \lambda 3968) \\
\left(L_{\odot}\right)\end{array}$ & & \\
\hline 1 & DP Tau & $\ldots$ & -1.74 & -1.73 & -1.69 & -2.17 & -1.74 & -1.55 & -1.91 & -1.95 & -1.87 & -1.35 & -1.46 & -1.69 & -9.07 \\
\hline 2 & CX Tau & $\ldots$ & $\ldots$ & -2.44 & -2.32 & -2.62 & $\ldots$ & -2.66 & -3.12 & $\mathrm{n}$ & $\mathrm{n}$ & $\ldots$ & $\ldots$ & -2.56 & -9.37 \\
\hline 3 & FP Tau & $\ldots$ & -2.32 & -2.32 & -2.15 & -2.16 & $\ldots$ & -2.24 & -2.53 & -2.23 & -2.31 & $\ldots$ & $\ldots$ & -2.27 & -9.17 \\
\hline 4 & FN Tau & $\ldots$ & -1.50 & -1.47 & -1.48 & -1.87 & -1.58 & -1.44 & -1.33 & -1.12 & -1.92 & $\ldots$ & $\ldots$ & -1.53 & -7.97 \\
\hline 5 & V409 Tau & $\ldots$ & $\mathrm{n}$ & $\mathrm{n}$ & $\mathrm{n}$ & $\mathrm{a}$ & $\mathrm{n}$ & $\mathrm{n}$ & $\mathrm{n}$ & $\mathrm{n}$ & $\mathrm{n}$ & -1.53 & -1.79 & -1.64 & -8.44 \\
\hline 6 & BP Tau & -0.99 & -1.16 & -1.19 & -1.16 & -1.24 & -1.25 & -1.24 & -1.22 & -1.18 & -1.14 & -1.13 & -1.02 & -1.17 & -8.14 \\
\hline 7 & DK Tau A & -0.67 & -0.89 & -0.87 & -0.76 & -1.18 & -0.79 & -0.98 & -0.74 & -0.72 & -0.74 & -0.76 & -0.64 & -0.79 & -7.86 \\
\hline 8 & HN Tau A & $\ldots$ & -1.10 & -1.26 & -1.00 & -1.92 & -0.54 & -0.78 & -1.46 & -0.85 & -1.10 & -0.89 & -0.67 & -0.93 & -8.37 \\
\hline 9 & UX Tau A & $\ldots$ & $\mathrm{n}$ & $\mathrm{n}$ & $\mathrm{n}$ & -1.56 & $\mathrm{n}$ & $\mathrm{n}$ & $\mathrm{n}$ & $\mathrm{n}$ & $\mathrm{n}$ & -1.16 & -1.46 & -1.51 & -8.81 \\
\hline 10 & GK Tau A & $\ldots$ & -1.31 & -1.56 & -1.34 & -1.64 & -1.41 & -1.47 & -1.16 & $\mathrm{n}$ & -1.30 & -0.76 & -0.88 & -1.38 & -8.35 \\
\hline 11 & GI Tau & $\ldots$ & -0.55 & -0.59 & -0.89 & -1.46 & -0.71 & -0.68 & -0.78 & -0.75 & -0.64 & $\ldots$ & $\ldots$ & -0.69 & -7.61 \\
\hline 12 & DM Tau & $\ldots$ & -1.92 & -1.94 & -1.89 & -1.92 & -2.43 & -1.91 & -1.91 & -2.33 & -1.93 & $\ldots$ & $\ldots$ & -1.92 & -8.79 \\
\hline 13 & LkCa 15 & $\ldots$ & -1.70 & -1.88 & -1.84 & -1.65 & -1.66 & -1.78 & -1.51 & $\mathrm{n}$ & -1.22 & $\ldots$ & $\ldots$ & -1.70 & -8.75 \\
\hline 14 & DS Tau & $\ldots$ & -1.30 & -1.20 & -1.45 & -1.67 & -1.28 & -1.39 & -1.12 & -0.93 & -1.13 & -1.76 & -1.51 & -1.28 & -8.42 \\
\hline 15 & SZ 65 & -1.92 & -1.87 & -2.21 & $\mathrm{a}$ & $\ldots$ & $\mathrm{n}$ & $\mathrm{n}$ & -2.76 & $\ldots$ & $\mathrm{n}$ & -1.66 & -1.88 & -1.94 & -8.90 \\
\hline 16 & SZ 68A & $\mathrm{n}$ & $\mathrm{n}$ & $\mathrm{n}$ & $\mathrm{n}$ & $\ldots$ & $\mathrm{n}$ & $\mathrm{n}$ & $\mathrm{n}$ & $\ldots$ & $\mathrm{n}$ & -1.16 & -1.20 & -1.18 & -8.13 \\
\hline 17 & SZ 73 & -1.14 & -1.21 & -1.29 & -1.33 & $\ldots$ & -1.22 & -1.30 & -1.41 & $\ldots$ & -1.27 & -1.16 & -1.05 & -1.22 & -8.54 \\
\hline 18 & HM Lup & -1.54 & -1.62 & -1.64 & -1.43 & $\ldots$ & -1.69 & -1.66 & -1.52 & $\ldots$ & -1.98 & -1.23 & -1.29 & -1.61 & -8.45 \\
\hline 19 & GW Lup & -1.61 & -1.74 & -1.80 & -1.87 & $\ldots$ & -1.84 & -1.99 & -1.91 & $\ldots$ & -1.94 & -1.89 & -1.96 & -1.87 & -8.71 \\
\hline 20 & GQ Lup & -0.32 & -0.49 & -0.66 & -0.40 & $\ldots$ & -0.53 & -0.61 & -0.26 & $\ldots$ & -0.51 & -0.10 & -0.11 & -0.36 & -7.39 \\
\hline 21 & SZ 76 & -1.92 & -2.12 & -2.16 & -2.52 & $\ldots$ & -2.82 & -2.82 & -3.46 & $\ldots$ & $\mathrm{n}$ & -1.85 & -2.22 & -2.23 & -8.96 \\
\hline 22 & RU Lup & -0.34 & -0.16 & 0.03 & -0.01 & $\ldots$ & b & b & 0.11 & $\ldots$ & -0.32 & 0.18 & 0.13 & -0.01 & -6.76 \\
\hline 23 & IM Lup & -1.68 & -1.92 & $\mathrm{a}$ & $\mathrm{a}$ & $\ldots$ & $\mathrm{n}$ & $\mathrm{n}$ & -2.41 & $\ldots$ & $\mathrm{n}$ & -1.49 & -1.72 & -1.75 & -8.68 \\
\hline 24 & RY Lup & $\mathrm{n}$ & $\mathrm{n}$ & $\mathrm{n}$ & $\mathrm{n}$ & $\ldots$ & $\mathrm{n}$ & $\mathrm{n}$ & $\mathrm{n}$ & $\ldots$ & $\mathrm{n}$ & -1.39 & -1.40 & -1.40 & -8.59 \\
\hline 25 & SZ 102 & -2.00 & -1.92 & -1.91 & -1.71 & $\ldots$ & -1.81 & -1.55 & -2.26 & $\ldots$ & -1.91 & -1.80 & -1.57 & -1.89 & \\
\hline 26 & SZ 111 & -1.49 & -1.65 & -1.70 & -1.86 & $\ldots$ & -1.67 & -1.82 & -1.87 & $\ldots$ & -1.69 & -1.99 & -1.89 & -1.74 & -8.79 \\
\hline 27 & SZ 98 & -1.39 & -1.66 & -1.66 & -1.53 & $\ldots$ & -1.56 & -1.80 & -1.65 & $\ldots$ & -1.50 & -1.42 & -1.36 & -1.53 & -8.58 \\
\hline 28 & EX Lup & 0.39 & 0.53 & 0.79 & 0.62 & $\ldots$ & $\mathrm{b}$ & 0.89 & 0.39 & $\ldots$ & 0.19 & 0.99 & 0.99 & 0.72 & -6.12 \\
\hline 29 & As $205 \mathrm{~A}$ & -0.44 & -0.26 & -0.20 & -0.10 & $\ldots$ & -0.05 & -0.39 & 0.02 & $\ldots$ & -0.30 & 0.23 & 0.20 & -0.07 & -6.58 \\
\hline 30 & DoAr 21 & $\ldots$ & $\ldots$ & $\ldots$ & $\mathrm{n}$ & $\ldots$ & $\ldots$ & $\mathrm{n}$ & $\mathrm{n}$ & $\ldots$ & $\mathrm{n}$ & $\ldots$ & $\ldots$ & & \\
\hline 31 & DoAr 24E & $\ldots$ & $\ldots$ & $\ldots$ & $\mathrm{n}$ & $\ldots$ & $\ldots$ & $\mathrm{n}$ & $\mathrm{n}$ & $\ldots$ & $\mathrm{n}$ & $\ldots$ & $\ldots$ & & \\
\hline 32 & DoAr 44 & -0.64 & -0.87 & -0.91 & -0.66 & $\ldots$ & -1.04 & -1.30 & -0.70 & $\ldots$ & -1.20 & -0.42 & -0.41 & -0.73 & -8.05 \\
\hline 33 & SR 21A & $\mathrm{n}$ & $\mathrm{n}$ & $\mathrm{n}$ & $\mathrm{n}$ & $\ldots$ & $\mathrm{n}$ & $\mathrm{n}$ & $\mathrm{n}$ & $\ldots$ & $\mathrm{n}$ & $\mathrm{n}$ & $\mathrm{n}$ & & \\
\hline 34 & V853 Oph & -1.21 & -1.43 & -1.50 & -1.48 & $\ldots$ & -1.41 & -1.39 & -1.38 & $\ldots$ & -1.43 & -1.65 & -1.54 & -1.46 & -8.08 \\
\hline 35 & RNO 90 & -0.01 & 0.13 & -0.01 & 0.02 & $\ldots$ & 0.32 & 0.12 & 0.05 & $\ldots$ & $\mathrm{n}$ & $\ldots$ & $\ldots$ & 0.06 & -7.26 \\
\hline 36 & V2508 Oph & -0.56 & -0.63 & -0.70 & -0.61 & $\ldots$ & -0.76 & -0.89 & -0.74 & $\ldots$ & -0.86 & -0.56 & -0.50 & -0.66 & -7.35 \\
\hline 37 & V1121 Oph & -1.01 & -1.39 & -1.47 & -1.06 & $\ldots$ & -1.35 & -1.56 & -1.10 & $\ldots$ & -1.00 & -0.90 & -0.95 & -1.12 & -8.30 \\
\hline 38 & $\mathrm{~J} 1842$ & -1.09 & -1.34 & -1.42 & -1.11 & $\ldots$ & -1.33 & -1.69 & -1.08 & $\ldots$ & -1.18 & -0.91 & -1.11 & -1.18 & -8.52 \\
\hline 39 & J1852 & -1.62 & -1.83 & -1.94 & -1.61 & $\ldots$ & -1.82 & $\mathrm{n}$ & -1.69 & $\ldots$ & -1.49 & -1.66 & -1.75 & -1.69 & -9.03 \\
\hline 40 & VV CrA & -0.22 & 0.24 & 0.25 & 0.12 & $\ldots$ & $\mathrm{b} ?$ & -0.12 & -0.65 & $\ldots$ & $\mathrm{n}$ & 1.07 & 0.70 & 0.21 & -6.43 \\
\hline 41 & SCrA A + B & $\mathrm{a}$ & $\mathrm{a}$ & $\mathrm{a}$ & $\mathrm{a}$ & $\ldots$ & b & -0.75 & 0.14 & $\ldots$ & -0.59 & $\mathrm{a}$ & $\mathrm{a}$ & -0.66 & -7.43 \\
\hline 42 & TW Hya & -1.43 & -1.48 & -1.42 & -1.25 & $\ldots$ & -1.68 & -1.64 & -1.49 & $\ldots$ & -1.64 & -1.83 & -1.79 & -1.53 & -8.67 \\
\hline 43 & TWA 3A & -3.51 & -3.70 & -3.54 & -3.58 & $\ldots$ & -3.87 & -3.84 & -3.47 & $\ldots$ & $\mathrm{n}$ & -3.18 & -3.18 & -3.48 & -10.15 \\
\hline 44 & V1057 Cyg ${ }^{c}$ & $\mathrm{n}$ & $\mathrm{n}$ & $\mathrm{n}$ & $\mathrm{n}$ & $\ldots$ & $\mathrm{n}$ & $\mathrm{n}$ & $\mathrm{n}$ & $\ldots$ & $\mathrm{n}$ & $\mathrm{a}$ & $\mathrm{n}$ & & $\sim-4.3$ \\
\hline 45 & V1515 $\mathrm{Cyg}^{\mathrm{c}}$ & $\mathrm{n}$ & $\mathrm{n}$ & $\mathrm{n}$ & $\mathrm{n}$ & $\ldots$ & $\mathrm{n}$ & $\mathrm{n}$ & $\mathrm{n}$ & $\ldots$ & $\mathrm{n}$ & 1.09 & $\mathrm{a}$ & & $\sim-4.5$ \\
\hline 46 & HD 143006 & $\mathrm{n}$ & $\mathrm{n}$ & $\mathrm{n}$ & -0.68 & $\ldots$ & $\mathrm{n}$ & $\mathrm{n}$ & $\mathrm{n}$ & $\ldots$ & $\mathrm{n}$ & -0.65 & $\mathrm{~b}$ & -0.66 & -7.99 \\
\hline
\end{tabular}


Table 5

(Continued)

\begin{tabular}{|c|c|c|c|c|c|c|c|c|c|c|c|c|c|c|c|}
\hline \multirow[b]{2}{*}{ ID } & \multirow[b]{2}{*}{ Name } & \multicolumn{12}{|c|}{$\log L_{\text {acc }}$} & \multirow[b]{2}{*}{$\begin{array}{l}\log \overline{L_{\text {acc }}} \\
\left(L_{\odot}\right)\end{array}$} & \multirow[b]{2}{*}{$\begin{array}{l}\log \dot{M}_{\mathrm{acc}} \\
\left(M_{\odot} \mathrm{yr}^{-1}\right)\end{array}$} \\
\hline & & $\begin{array}{l}(\mathrm{H} \zeta) \\
\left(L_{\odot}\right)\end{array}$ & $\begin{array}{l}(\mathrm{H} \delta) \\
\left(L_{\odot}\right)\end{array}$ & $\begin{array}{l}(\mathrm{H} \gamma) \\
\left(L_{\odot}\right)\end{array}$ & $\begin{array}{l}(\mathrm{H} \beta) \\
\left(L_{\odot}\right)\end{array}$ & $\begin{array}{l}(\mathrm{H} \alpha) \\
\left(L_{\odot}\right)\end{array}$ & $\begin{array}{c}(\mathrm{He} \text { I } \lambda 4026) \\
\left(L_{\odot}\right)\end{array}$ & $\begin{array}{c}\text { (He I } \lambda 4471) \\
\left(L_{\odot}\right)\end{array}$ & $\begin{array}{c}(\text { He I } \lambda 5876) \\
\left(L_{\odot}\right)\end{array}$ & $\begin{array}{c}(\mathrm{He} \text { I } \lambda 6678) \\
\left(L_{\odot}\right)\end{array}$ & $\begin{array}{c}\text { (He II } \lambda 4686) \\
\left(L_{\odot}\right)\end{array}$ & $\begin{array}{c}(\mathrm{Ca} \text { II } \lambda 3934) \\
\left(L_{\odot}\right)\end{array}$ & $\begin{array}{c}\text { (Ca II } \lambda 3968) \\
\left(L_{\odot}\right)\end{array}$ & & \\
\hline 47 & DI Cep & -0.05 & -0.04 & 0.19 & 0.53 & $\ldots$ & $\mathrm{n}$ & 0.04 & 0.27 & $\ldots$ & -0.24 & 0.54 & 0.45 & 0.26 & -6.97 \\
\hline 48 & As $353 \mathrm{~A}$ & $\mathrm{n}$ & $\mathrm{a}$ & $\mathrm{a}$ & $\mathrm{a}$ & $\ldots$ & $\mathrm{n}$ & 0.08 & -0.22 & $\ldots$ & $\mathrm{n}$ & -0.35 & $\mathrm{a}$ & -0.13 & -6.81 \\
\hline
\end{tabular}

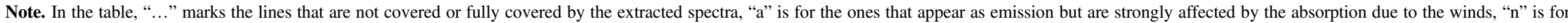
those that do not clearly appear as emission lines, and "b" is for the ones that are seriously contaminated by other nearby emission lines.

${ }^{c}$ For the two FU Ori objects, their accretion rates are obtained from Green et al. (2006) by fitting the SEDs, assuming a central stellar mass of $1 M_{\odot}$. 

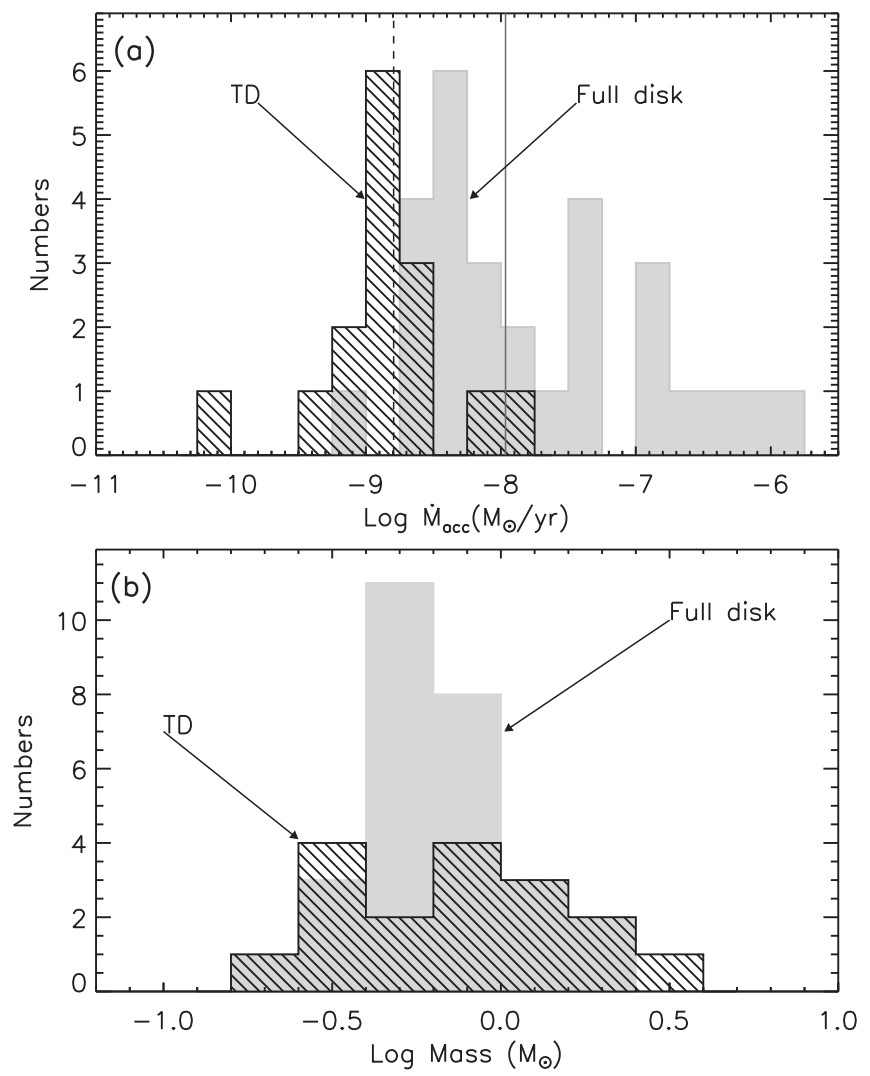

Figure 7. (a) Distribution of accretion rates for full disks (gray-filled histogram) and TDs (hatch-filled histogram). The solid line and the dashed line mark the median accretion rates of full disks and TDs, respectively. (b) Distribution of stellar masses for full disks (gray-filled histogram) and TDs (hatch-filled histogram).

forbidden-line profiles demonstrate that the inner gaseous disk of $\mathrm{LkCa} 15$ is in a more evolved stage than that of J1842.

\subsection{Mass Outflow Rates}

Previous analysis of [O I] $\lambda 6300$ high-resolution spectra has demonstrated that a significant number of LVC-BC and LVCNC peak centroids are blueshifted with respect to the stellar velocity (Simon et al. 2016; Banzatti et al. 2018; McGinnis et al. 2018). With the largest blueshifts found in sources surrounded by lower-inclination disks and an emitting region within $\sim 0.5$ au from the star, Simon et al. (2016) attributed the LVC-BC to the base of an MHD-driven wind. Although the focus of our paper is on forbidden-line luminosities and line ratios, here we show that the kinematics of the LVC-BC and LVC-NC of our sample are consistent with previous findings.

The top panel of Figure 11 shows the distribution of the NC and BC centroids. About 63\% (15/24) of NCs and 57\% (13/23) of BCs have blueshifts larger than $1.5 \mathrm{~km} \mathrm{~s}^{-1}$, while only $8 \%$ $(2 / 24)$ of $\mathrm{NCs}$ and $22 \%(5 / 23)$ of BCs are redshifted by more than $1.5 \mathrm{~km} \mathrm{~s}^{-1}$. The larger proportion of blueshifts when compared to no shift or redshifts is consistent with a wind origin for both components. The bottom panel of Figure 11 shows the relation between disk inclination and the BC and NC FWHMs, corrected for instrumental broadening and normalized by stellar mass.

If we attribute the line width primarily to Keplerian broadening as in previous studies (Simon et al. 2016; McGinnis et al. 2018), most BCs trace radii within $0.5 \mathrm{au}$, while NCs probe gas farther out but mostly within $\sim 5$ au. A few sources appear as outliers in this plot and are worth discussing. First, the FWHMs from DK Tau A and RU Lup are much larger than those expected from pure Keplerian rotation using the disk inclinations given in Table 1. The disk inclination of DK Tau A is not well constrained: the $20^{\circ}$ value we adopted here comes from the analysis of millimeter $\mathrm{CO}$ lines, but the continuum emission points to a much higher inclination of $65^{\circ}$ (Simon et al. 2017). In the case of RU Lup the outer disk inclination of $3^{\circ}$ derived from ALMA millimeter imagery may not apply to the forbidden lines studied here. Indeed, spectroastrometry in the $\mathrm{CO}$ rovibrational band suggests a higher inclination of $35^{\circ}$ for the inner disk (Pontoppidan et al. 2011), and an even higher inclination is implied by MIDI visibilities (see Varga et al. 2018 and Banzatti et al. 2018 for further details). If we use these alternative/higher disk inclinations, the BC FWHM of DK Tau A falls in the same region as the other BCs, and that of RU Lup becomes much closer to that region. Finally, the BCs from LkCa 15, SZ 73, RNO 90, and VV CrA fall within the domain of LVC-NC; they could have been misclassified using our stringent cut in FWHM that does not take into account disk inclination.

Having established that our sample supports the scenario in which LVC-BC and LVC-NC trace a wind and their FWHMs are consistent with being broadened by Keplerian rotation, we use the [O I] $\lambda 6300$ luminosity and our constraints on the gas temperature, velocity, and emitting radii to compute wind mass-loss rates. Figure 6(a) shows that electron densities $n_{e} \geqslant 3 \times 10^{6} \mathrm{~cm}^{-3}$ are needed to reproduce the measured LVC line ratios. As these densities are larger than the [O I] $\lambda 6300$ critical density, which is $1.8 \times 10^{6} \mathrm{~cm}^{-3}$, the LVC emitting gas is most likely in LTE. In addition, very high densities are required to make the [O I] $\lambda 6300$ line optically thick (see Table 9 in Hollenbach \& McKee 1989). Hence, we will use equations for optically thin LTE gas to relate the LVC [O I] $\lambda 6300$ luminosity to a gas mass. As a shock origin is more likely for the HVC emission and this lower-density gas may not be in LTE (see Section 4.2.2 and Figure 6(b)), we will use equations derived for radiative shocks to estimate the mass-loss rate from this HVC.

\subsubsection{Mass-Loss Rates from the LVC}

As the [O I] $\lambda 6300$ line is optically thin, its luminosity $\left(L_{6300}\right)$ can be written as

$$
L_{6300}=N_{\mathrm{u}} A h \nu,
$$

where $N_{\mathrm{u}}$ is the total number of $\mathrm{O}$ atoms in the upper ${ }^{1} D_{2}$ level, $A$ is the transition probability $\left(6.503 \times 10^{-3} \mathrm{~s}^{-1}\right)$, and $h \nu$ is the associated energy they emit (Dere et al. 1997). In addition, for gas in LTE the total number of $\mathrm{O}$ atoms $(N)$ is related to $N_{\mathrm{u}}$ as

$$
N_{\mathrm{u}}=N \frac{g_{u} e^{-h \nu / k T}}{Z(T)},
$$

where $g_{u}$ is the upper-level statistical weight, $k$ is the Boltzmann constant, $T$ is the gas temperature, and $Z(T)$ is the partition function at temperature $T$. We calculate $Z(T)$ assuming a five-level oxygen atom. Considering that in disks the abundance of neutral oxygen is reduced because half of the cosmic $\mathrm{O}$ is in silicate grains (Jenkins 2009) and the wind is launched from outside the dust sublimation radius (Figure 11 lower panel), we take $\alpha(\mathrm{O})=3.2 \times 10^{-4}$ to calculate the total 

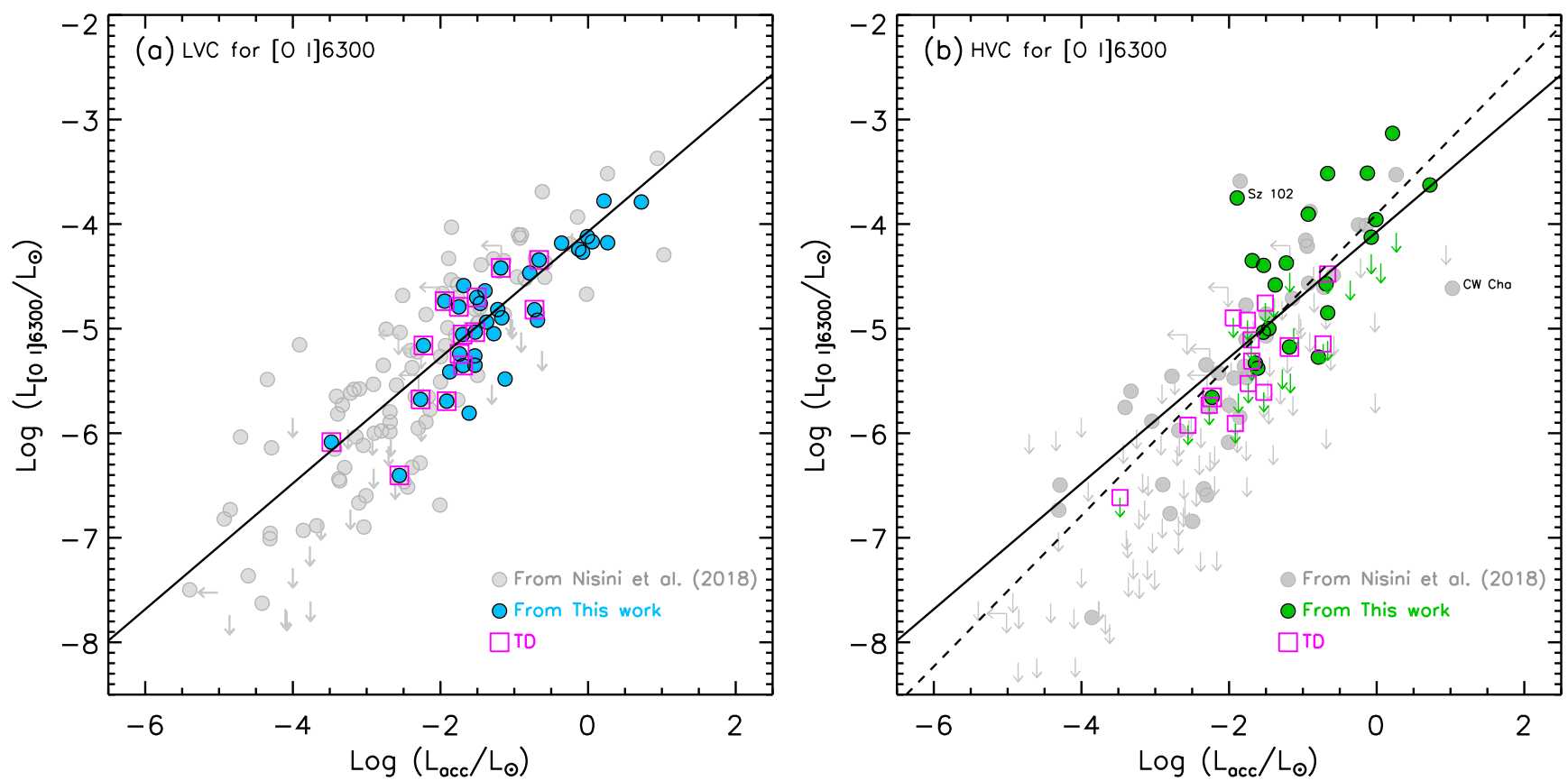

Figure 8. Line luminosities for the [O I] $\lambda 6300$ LVC (panel (a)) and for the HVC (panel (b)) vs. accretion luminosities. Magenta squares mark TDs, color-filled circles are sources from this work, and gray circles are additional sources from Nisini et al. (2018). Solid lines are the best linear fit to all LVC detections present in panel (a). The additional dashed line in panel (b) gives the best-fit linear relation to the detected HVCs. The upper limits in panel (b) for our sample are calculated assuming a Gaussian profile with an FWHM of $70 \mathrm{~km} \mathrm{~s}^{-1}$, a median of the HVC FWHM, and a peak of $3 \times$ rms.

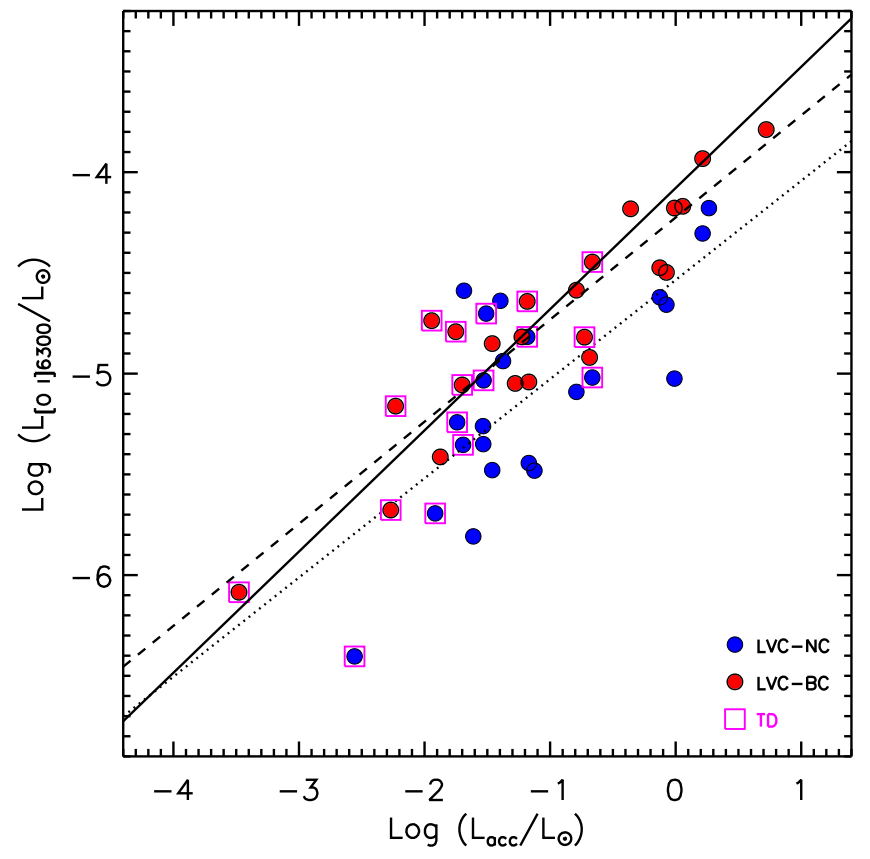

Figure 9. Line luminosities for [O I] $\lambda 6300$ LVC-NC (blue) and LVC-BC (red) vs. accretion luminosities. Magenta squares mark TDs. The solid line is the linear fit to the total LVC (see Figure 8(a)), while the dashed line and dotted line are the best fits to the LVC-BC and LVC-NC, respectively.

mass of gas $M$ from the total number of $\mathrm{O}$ I atoms. Finally, the mass-loss rate $\dot{M}_{\text {wind }}$ can be written as

$$
\dot{M}_{\text {wind }}=M \frac{V_{\text {wind }}}{l_{\text {wind }}}
$$

where $V_{\text {wind }}$ and $l_{\text {wind }}$ are the velocity and wind height, respectively. We further write the unconstrained wind height as
Table 6

Statistics on the Line Profiles

\begin{tabular}{lcccr}
\hline \hline & Only NC & Only BC & NC+BC & \multicolumn{1}{c}{ HVC } \\
\hline Full disks & $28 \%(8 / 29)$ & $24 \%(7 / 29)$ & $24 \%(7 / 29)$ & $72 \%(21 / 29)$ \\
TDs & $44 \%(7 / 16)$ & $44 \%(7 / 16)$ & $13 \%(2 / 16)$ & $13 \%(2 / 16)$
\end{tabular}

$l_{\text {wind }}=f \times r_{\text {base }}$, i.e., the wind vertical extent is $f$ times the emitting radius at the base of the wind. Combining Equations (6) through (8), we have

$$
\begin{aligned}
\dot{M}_{\text {wind }} & =\frac{V_{\text {wind }}}{l_{\text {wind }}} \frac{\eta m_{\mathrm{H}}}{\alpha(\mathrm{O})} e^{h \nu / k T} Z(T) \frac{L_{6300}}{g_{\mathrm{u}} A h \nu} \\
& =\mathrm{C}(\mathrm{T})\left(\frac{V_{\text {wind }}}{10 \mathrm{~km} \mathrm{~s}^{-1}}\right)\left(\frac{l_{\text {wind }}}{1 \mathrm{au}}\right)^{-1}\left(\frac{L_{6300}}{L_{\odot}}\right) M_{\odot} \mathrm{yr}^{-1},
\end{aligned}
$$

where $\eta=1.34$ is the ratio of total gas mass to hydrogen mass. From this equation it is clear that the estimated mass-loss rate has a strong dependence on the gas temperature $T$ and scales linearly with $V_{\text {wind }}$ and $l_{\text {wind }}$. LVC gas temperatures range from 5000 to $10,000 \mathrm{~K}$ (Section 4.2.1), and within this range $C(T)$ varies by an order of magnitude: from $2.4 \times 10^{-4}$ for $T=5000 \mathrm{~K}$ down to $2.6 \times 10^{-5}$ for $T=10,000 \mathrm{~K}$.

For each source with an [O I] $\lambda 6300$ detection we calculate its own $\dot{M}_{\text {wind }}$ by taking as $V_{\text {wind }}$ the median of projected peak velocities and as $r_{\text {base }}$ the Keplerian radius from half of the line FWHM, corrected for the instrumental broadening and projected (see Figure 11, bottom panel). For the BC these values correspond to $-15 \mathrm{~km} \mathrm{~s}^{-1}$ and $0.15 \mathrm{au}$, while for the NC they are $-6 \mathrm{~km} \mathrm{~s}^{-1}$ and $1.7 \mathrm{au} .{ }^{18}$ We then divide $\dot{M}_{\text {wind }}$ by each

\footnotetext{
$\overline{18}$ Unprojected values for the $\mathrm{BC}$ are $-12 \mathrm{~km} \mathrm{~s}^{-1}$ and $0.33 \mathrm{au}$, while for the $\mathrm{NC}$ they are $-4 \mathrm{~km} \mathrm{~s}^{-1}$ and $4.3 \mathrm{au}$.
} 

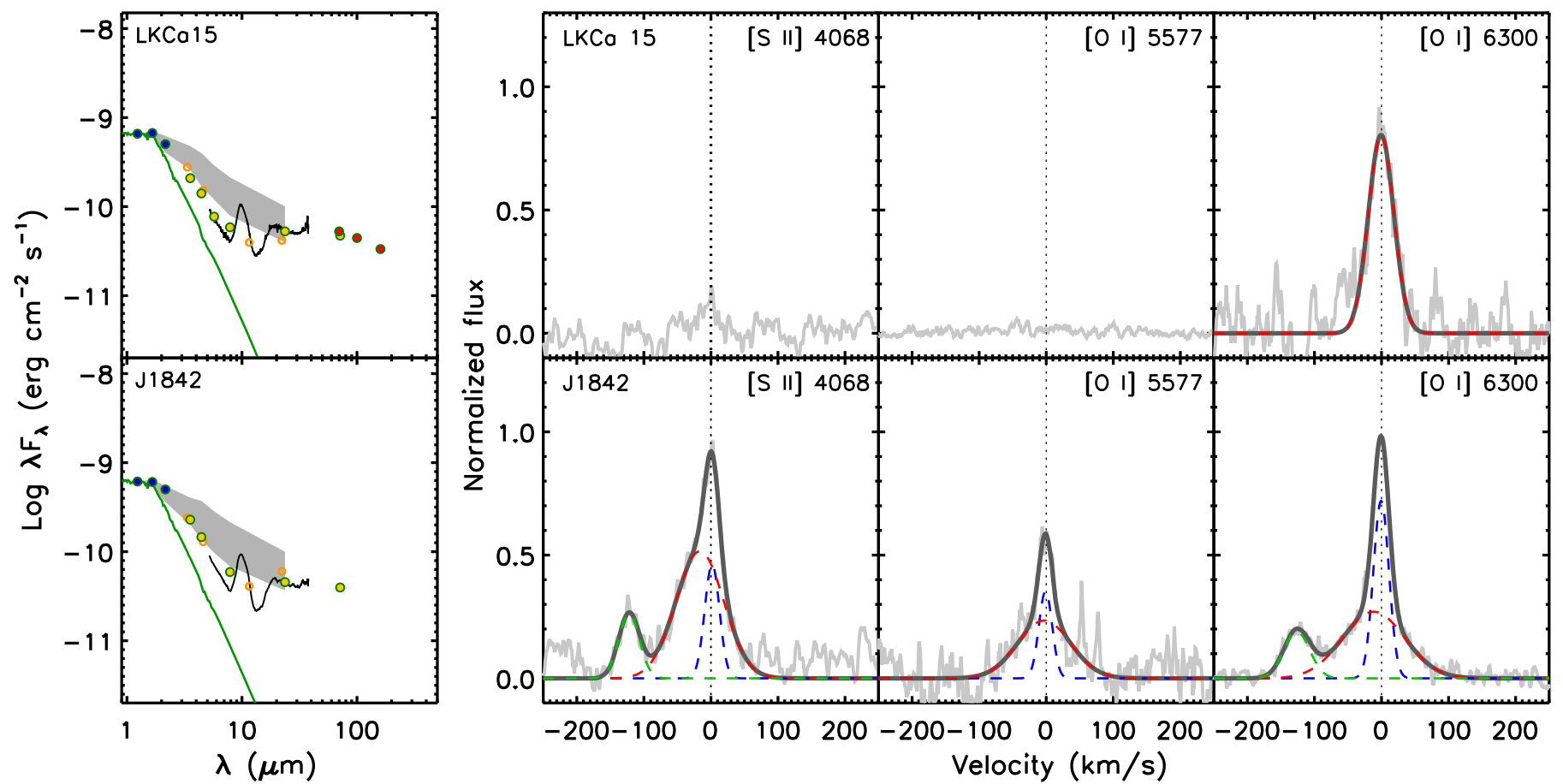

Figure 10. Left panels: SEDs of two TDs, LkCa 15 and J1842, around stars of similar spectral type and accretion rate. In each panel, the gray region shows the upper and lower quartiles of the Lynds 1641 CTTS median SED with the same spectral type. The median SED has been reddened with the extinction of each source and then normalized to the $J$-band flux. Right panels: [S II] $\lambda 4068$, [O I] $\lambda 5577$, and [O I] $\lambda 6300$ line profiles from the same sources. While these systems have very similar SEDs, the forbidden-line profiles suggest that the inner gaseous disk of LkCa 15 is in a more evolved stage than that of J1842.

source $\dot{M}_{\text {acc }}$ and show in Figure 12 how the median and upperand lower-quartile ratios depend on $T$ and $l_{\text {wind }}$. As expected from Equation (9), $\dot{M}_{\text {wind }}$, and hence $\dot{M}_{\text {wind }} / \dot{M}_{\text {acc }}$ ratios, increase with decreasing gas temperature and wind height. Since the extent of the [O I] $\lambda 6300$ emitting region is unknown, we vary the factor $f$ within a range of plausible values for which $l_{\text {wind }}$ is similar in the LVC-BC and LVC-NC.

For the BC, $f=1$ produces unreasonably large $\dot{M}_{\text {wind }} / \dot{M}_{\text {acc }}$ ratios for most temperatures, except for $10,000 \mathrm{~K}$. However, such a high temperature is unlikely; otherwise, emission lines from ionized oxygen would have been also easily detected (see Section 5.7 in Simon et al. 2016). Heating processes not driven by photons (e.g., ambipolar drift heating; see Safier 1993) could result in higher gas temperatures without affecting the ionization state, but their efficiency has not been sufficiently explored in this context. We will further discuss the implications of Figure 12 in Section 6. However, it is already worth noting that, for likely wind extents, the mass-loss rates implied by the BC are much higher than those implied by the NC. This results from the fact that [O I] $\lambda 6300$ luminosities, as well as the median peak centroid, are larger for the LVC-BC than for the LVC-NC (see Figures 9 and 11), and the wind heights of the $\mathrm{NC}$ are likely higher than for the $\mathrm{BC}$.

This last point is important; hence, we summarize here additional arguments supporting a large vertical extent of the LVC-NC. First, it is highly unlikely that the wind height of the LVC-NC is small $(f<1)$ compared to the Keplerian radius because with our high-resolution $\left(\Delta v \sim 6 \mathrm{~km} \mathrm{~s}^{-1}\right)$ spectra we would see double-peaked NC profiles. Next, as we will discuss, it is very likely that the extent of the NC is larger than that of the BC. The LVC-BC traces an MHD wind because of its small Keplerian radius (0.05-0.5 au) and blueshifted centroids; thermal pressure is insufficient to launch the gas flow, and
MHD forces are required. The NC may similarly also trace an MHD wind and being further out than the $\mathrm{BC}$ is likely to be more vertically extended. If the LVC-NC is partly supported by a photoevaporative (thermal) wind, it should be even more extended vertically given the gas temperature $(5000-10,000 \mathrm{~K})$ inferred from the line ratios (see Figure 6). As an example, in the X-ray-driven photoevaporative wind model (Ercolano \& Owen 2016), the winds can extend to about 35 au above the disk. While the wind height cannot be determined without spatially resolved observations, our finding that the mass-loss rates implied by the $\mathrm{BC}$ are higher than those implied by the NC should be reliable.

\subsubsection{Mass-loss Rates from the HVC}

As discussed in Section 4.2.2, shock models reproduce most of the [O I] and [S II] HVC line ratios. In fast shocks, most of the mechanical energy of the pre-shock gas is converted into heat (e.g., Hollenbach \& McKee 1979; Shull \& McKee 1979). The mass-loss rate can then be estimated assuming that a certain fraction of the kinetic energy is radiated away in the [O I] $\lambda 6300$ line. Hence, we use the equations for radiative shocks as presented, e.g., in Hollenbach \& Gorti (2009). If the hydrogen density is high $\left(n_{\mathrm{H}} \sim 10^{6} \mathrm{~cm}^{-3}\right)$, the line luminosity can be computed as

$$
L_{6300}=\frac{y_{0}}{2} \dot{M}_{\text {loss }} V_{\mathrm{s}}^{2},
$$

with $y_{0}$ being the fraction of cooling that goes into the [O I] $\lambda 6300$ line and $V_{\mathrm{s}}$ being the shock velocity. The mass-loss rate is then

$$
\dot{M}_{\mathrm{jet}}=L_{6300} \frac{2}{y_{0}} V_{\mathrm{s}}^{-2} .
$$



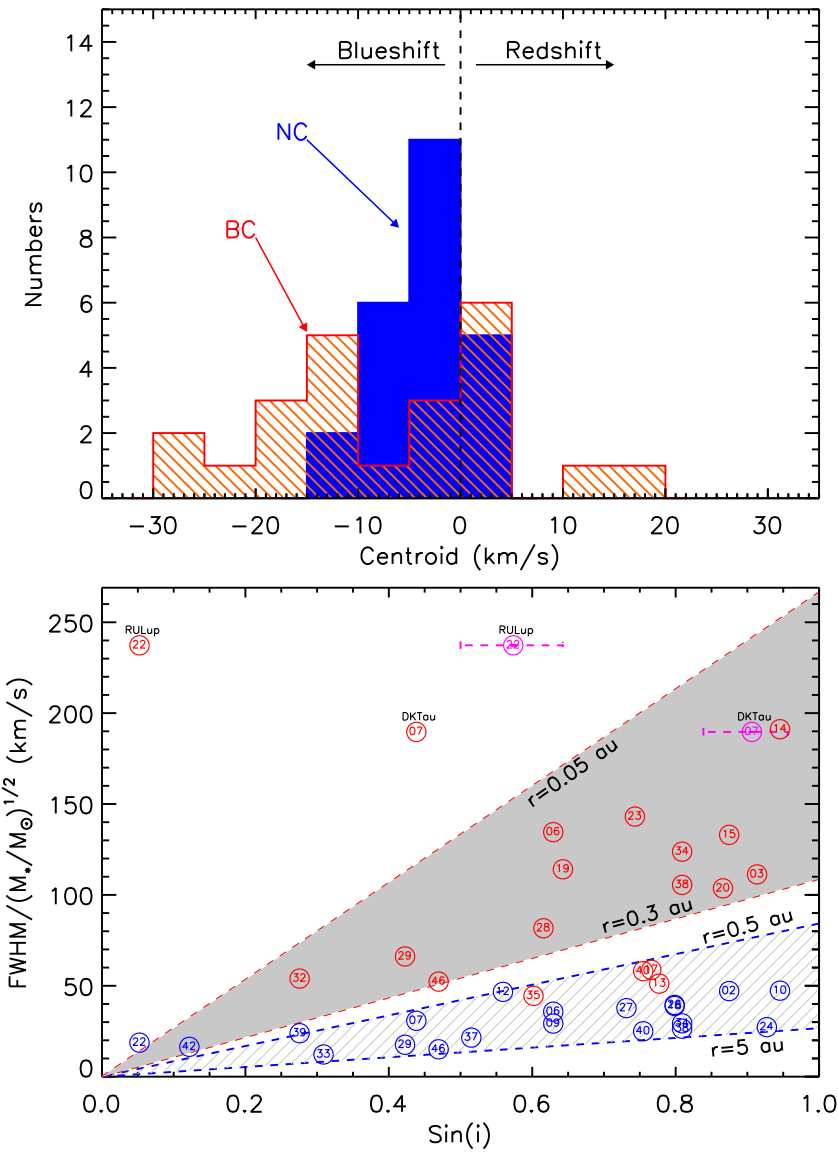

Figure 11. Top panel: distribution of centroids for the LVC-NC (blue-filled histogram) and the LVC-BC (hatch-filled histogram). Note that many centroids are blueshifted with respect to the stellar velocity (see also Banzatti et al. 2018 for more details on the [O I] kinematics). Bottom panel: FWHM corrected for instrumental broadening and normalized by the stellar mass vs. disk inclination, LVC-BC in red and LVC-NC in blue. Dashed lines show Keplerian FWHM as a function of disk inclination at radii of $0.05,0.3,0.5$, and $5 \mathrm{au}$. The hatch-filled area and gray-filled area enclose most of the $\mathrm{NC}$ and $\mathrm{BC}$, respectively. The magenta circles locate two sources (RU Lup, ID 22, and DK Tau A, ID 7) for which we also use alternative disk inclinations.

At a density $n_{\mathrm{H}} \sim 10^{6} \mathrm{~cm}^{-3}, y_{0}$ is around $0.1-0.3$; hence, we take here $y_{0}=0.2$. As for the $\mathrm{LVC}$, we first compute individual $\dot{M}_{\text {jet }}$, divide them by each source $\dot{M}_{\text {acc }}$, and show the behavior of median and upper- and lower-quartile ratios for a range of shock velocities compatible with observations (e.g., Hartigan et al. 1987). The faster the shock velocity, the lower the $\dot{M}_{\text {jet }} / \dot{M}_{\text {acc }}$ ratio. For a typical shock velocity of $30 \mathrm{~km} \mathrm{~s}^{-1}$ (Hartigan et al. 1994), the median $\dot{M}_{\text {jet }} / \dot{M}_{\text {acc }}$ ratio is around 0.1 .

The approach we have taken above differs from the one typically used in the literature, where it is assumed that the jet has uniform properties within the slit width and the line luminosity is calculated from a collisional excitation model akin to that described in Section 4.2.1 for the LVC (see also Figure 6, left panel). More specifically, and following Hartigan et al. (1995) and Nisini et al. (2018), the mass-loss rate from the jet can be written as

$$
\dot{M}_{\text {jet }}=C\left(T, n_{\mathrm{e}}\right)\left(\frac{V_{\perp}}{100 \mathrm{~km} \mathrm{~s}^{-1}}\right)\left(\frac{l_{\perp}}{100 \mathrm{au}}\right)^{-1}\left(\frac{L_{6300}}{L_{\odot}}\right) M_{\odot} \mathrm{yr}^{-1},
$$

where $V_{\perp}$ is the component of the jet velocity in the plane of the sky (we assume $100 \mathrm{~km} \mathrm{~s}^{-1}$ as Nisini et al. 2018) and $l_{\perp}$ is the projected size of the slit aperture $(0$ " 861$)$ on the plane of the sky. $C\left(T, n_{\mathrm{e}}\right)$ depends on the gas temperature and electron density and is calculated assuming a five-level oxygen atom as in Section 4.2.1. For $T=10,000 \mathrm{~K}, C\left(T, n_{\mathrm{e}}\right)$ are $9.0 \times 10^{-5}$, $6.1 \times 10^{-5}$, and $4.6 \times 10^{-5}$ for $n_{\mathrm{e}}=5.0 \times 10^{4}, 7.5 \times 10^{4}$, and $1.0 \times 10^{5} \mathrm{~cm}^{-3}$, respectively. Taking the same temperature $(10,000 \mathrm{~K})$ and electron density $\left(5.0 \times 10^{4} \mathrm{~cm}^{-3}\right)$ as Nisini et al. (2018), we find a median $\dot{M}_{\text {jet }} / \dot{M}_{\text {acc }}$ ratio of $\sim 0.1$. The right panel of Figure 13 also shows the $\dot{M}_{\text {jet }} / \dot{M}_{\text {acc }}$ for the [O I] $\lambda 6300$ detected HVCs in Nisini et al. (2018) after implementing the new Gaia distances (Bailer-Jones et al. 2018) and assuming $\alpha(\mathrm{O})=$ $3.2 \times 10^{-4}, T=10,000 \mathrm{~K}$, and $n_{\mathrm{e}}=5.0 \times 10^{4} \mathrm{~cm}^{-3}$. The median $\dot{M}_{\text {jet }} / \dot{M}_{\text {acc }}$ ratio is $\sim 0.18$ for this sample. ${ }^{19}$ Furthermore, the same panel shows the dependence of $\dot{M}_{\text {jet }} / \dot{M}_{\text {acc }}$ on $T$ and $n_{\mathrm{e}}$ : the ratio decreases with increasing gas temperature and electron density. For values of $T \sim 7000-15,000 \mathrm{~K}$ and $n_{\mathrm{e}}=(2-6) \times 10^{4} \mathrm{~cm}^{-3}$ that are appropriate for the base of the T Tauri jets (Hartigan \& Morse 2007; Agra-Amboage et al. 2011; Giannini et al. 2013; Maurri et al. 2014), the median $\dot{M}_{\text {jet }} / \dot{M}_{\text {acc }}$ ratios vary from 0.8 to 0.04 .

\section{Discussion}

It is already well established that the HVC and the LVC of forbidden lines trace different physical environments, where the HVC is more spatially extended and formed in shock-excited collimated jets, and the LVC, with a higher density than the $\mathrm{HVC}$, is confined to scales of less than $5 \mathrm{au}$ (e.g., Hartigan et al. 1995; Hirth et al. 1997; Simon et al. 2016). Our study expands on the literature by combining the [S II] $\lambda 4068$ transition with the well-studied [O I] $\lambda 6300$ and [O I] $\lambda 5577$ lines, demonstrating that the same kinematic components appear in all three lines, thus enabling the use of component line ratios to constrain the properties of the emitting gas (Section 4.1 and Figure 5). By including detections and upper limits, we found that the HVC SII40/OI63 ratios are statistically higher than the LVC (both BC and $\mathrm{NC}$ ) and that there is a low probability that the $\mathrm{BC}$ OI55/63 ratios are drawn from the same parent population as the NC and HVC (Table 4). These differences and the mean line ratios of the three components corroborate previous suggestions, mostly based on kinematics (e.g., Simon et al. 2016; Banzatti et al. 2018; McGinnis et al. 2018), that the LVC-BC traces a higher density/hotter region than the LVC-NC, probably the base of an MHD disk wind. Finally, we could show that thermally excited gas with temperatures of $5000-10,000 \mathrm{~K}$ and electron densities of $\sim 10^{7}-10^{8} \mathrm{~cm}^{-3}$ can explain most of the LVC-BC and LVC-NC line ratios, while the HVC ratios are best reproduced by radiative shock models where forbidden lines arise in the hot $(\sim 7000-10,000 \mathrm{~K})$ but less dense (total $\mathrm{H}$ density $\sim 10^{6} \mathrm{~cm}^{-3}$ ) post-shock cooling zone (Sections 4.2 .1 and 4.2.2).

Armed with the physical properties of the emitting gas in each component, we computed mass-loss rates for the LVCBC, LVC-NC, and HVC (Section 5.4). To evaluate the efficiency of winds in removing disk mass, we also computed

\footnotetext{
19 The difference between our 0.18 value and the 0.07 value in Nisini et al. (2018) is due to the following: (1) we use a different oxygen abundance $\left(3.2 \times 10^{-4}\right.$ vs. $\left.4.6 \times 10^{-4}\right) ;(2)$ our value gives the median of the distribution, while Nisini et al. (2018) report the most common log $\dot{M}_{\text {jet }} / \dot{M}_{\text {acc }}$ value; and (3) we use the new Gaia DR2 distances.
} 

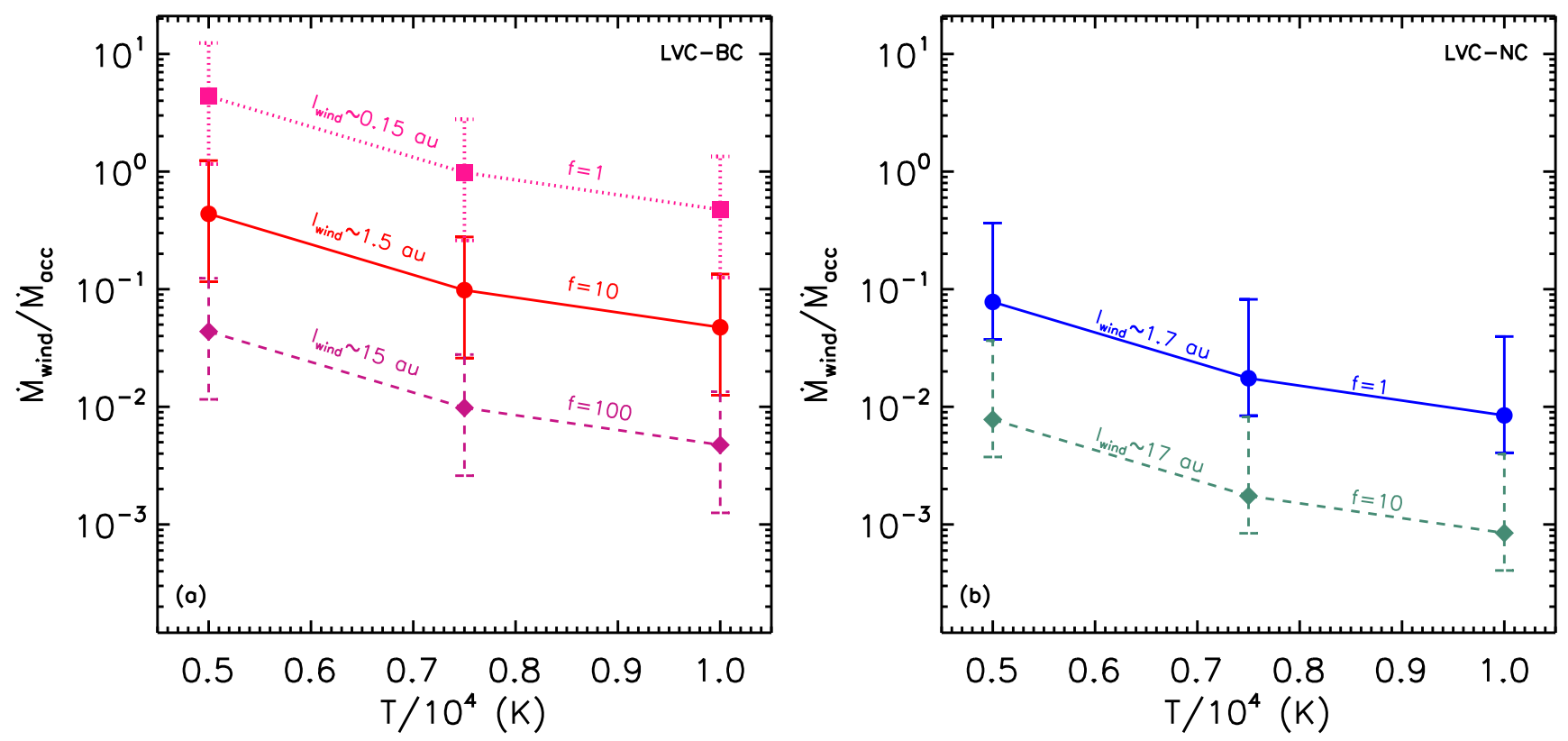

Figure 12. Median $\dot{M}_{\text {wind }} / \dot{M}_{\text {acc }}$ ratios for our sample as a function of gas temperature and for different wind heights $\left(l_{\text {wind }}=f \times r_{\text {base }}\right.$ ), for LVC-BC (left panel) and LVC-NC (right panel). In each panel, the error bars show the upper and lower quartiles of the $\dot{M}_{\text {wind }} / \dot{M}_{\text {acc }}$ ratio distributions.

mass accretion rates by converting the luminosity of several permitted lines covered in our spectra to the accretion luminosity (Section 5.1). For the HVC, we relied on radiative shock models that convert a fraction of the mechanical energy of the pre-shock gas into the [O I] $\lambda 6300$ luminosity. We found relatively low $\dot{M}_{\text {jet }} / \dot{M}_{\text {acc }}$ with a median value of $\sim 0.1$ for typical shock velocities of $30 \mathrm{~km} \mathrm{~s}^{-1}$, but this ratio could be as low as 0.01 for a high shock velocity of $100 \mathrm{~km} \mathrm{~s}^{-1}$ or as high as 0.3 for a low shock velocity of $20 \mathrm{~km} \mathrm{~s}^{-1}$. These values are similar to those previously reported in the literature, which were instead calculated assuming a collisional excitation model, and hence sensitive to several not well-constrained quantities such as gas temperature and electron density in addition to jet velocity (e.g., Hartigan et al. 1995; Nisini et al. 2018; see also Section 5.4.2).

A first estimate for mass-loss rates for the LVC, assuming a spherical outflow geometry, comes from Natta et al. (2014), who find $\dot{M}_{\text {wind }} / \dot{M}_{\text {acc }}$ between 0.1 and 1 for their sample of $\mathrm{T}$ Tauri stars in Lupus and $\sigma$ Ori. These values are on the high end of the HVC $\dot{M}_{\text {jet }} / \dot{M}_{\text {acc }}$ ratios, suggesting that the LVC may have a higher mass-loss rate than the jet. Our work is the first to provide $\dot{M}_{\text {wind }} / \dot{M}_{\text {acc }}$ separately for the $\mathrm{BC}$ and NC. One important finding is that the mass-loss rate from the $\mathrm{BC}$ exceeds that from the $\mathrm{NC}$ by at least a factor of 5 . A comparison of the mass-loss rates between the $\mathrm{BC}$ and $\mathrm{HVC}$ is more uncertain. To this end we explored the sensitivity of wind mass-loss rates in thermally excited gas to the parameters that are the least constrained and most affect the estimates, namely, the gas temperature and $l_{\text {wind }}$ (Section 5.4.1). For the BC, a wind extent equal to the radial extent inferred from Keplerian broadening of the [O I] $\lambda 6300$ lines $(f=1$, Figure 12, left panel) provides unreasonably large $\dot{M}_{\text {wind }} / \dot{M}_{\text {acc }}$, i.e., larger than 1 , for most sources. Most likely the vertical extent of the BC, which remains unconstrained from our data, is larger than the Keplerian radius inferred from the BC FWHM. If we assume a vertical extent comparable to the $\mathrm{NC}$ radius inferred from Keplerian broadening ( $f=1$, Figure 12, right panel; and $f=10$, Figure 12 , left panel), the median $\dot{M}_{\text {wind }} / \dot{M}_{\text {acc }}$ from the BC ranges from $\sim 0.05$ for gas as hot as $10,000 \mathrm{~K}$ up to $\sim 0.4$ for gas at $5000 \mathrm{~K}$. As already discussed in Section 5.4.1, a temperature of $10,000 \mathrm{~K}$ is unlikely for the LVC gas; hence, higher mass-loss rates are more realistic. However, given the sensitivity of $\dot{M}_{\text {wind }} / \dot{M}_{\text {acc }}$ on $l_{\text {wind }}$, we cannot conclusively state that the LVC-BC is carrying away more mass than the HVC. What is more certain is that the NC $\dot{M}_{\text {wind }} / \dot{M}_{\text {acc }}$ is lower than the $\mathrm{BC}$ for reasonable $l_{\text {winds }}$ by at least a factor of 5 . Thus, most of the disk mass appears to be lost close to the central star in the region traced by the $\mathrm{BC}$.

How do our results compare with theoretical models of winds? Both photoevaporative and MHD winds have been proposed to explain forbidden-line emission. However, as already pointed out by Simon et al. (2016) and confirmed by McGinnis et al. (2018) and Banzatti et al. (2018), the only component that may be tracing a thermal photoevaporative wind is the LVC-NC, as the BC is formed well inside the gravitational potential well of the star. Our findings that most $\mathrm{NC}$ ratios can be reproduced by thermally excited gas and that the line luminosity correlates with accretion luminosity agree with the most recent predictions from X-ray-driven photoevaporative winds (Ercolano \& Owen 2016). In these models, stellar EUV photons heat the forbidden-line-emitting region and, due to their low penetration depth, constrain it to a thin vertically extended (up to $\sim 35$ au) zone above the inner disk (see, e.g., their Figure 4). In addition, Ercolano \& Owen (2016) claim that most of the EUV luminosity comes from accretion and that would lead to a correlation between the [O I] luminosity and $L_{\text {acc }}$. The vertical extent of the emitting region results in a wide range of wind velocities that can broaden the [O I] lines up to $\sim 30 \mathrm{~km} \mathrm{~s}^{-1}$ for close to edge-on disks while keeping moderate blueshifts of up to $\sim 7 \mathrm{~km} \mathrm{~s}^{-1}$ for disks inclined by $\sim 50^{\circ}$ with respect to the observer. ${ }^{20}$ While the predicted FWHM and peak centroids are consistent with most

\footnotetext{
${ }^{20}$ Note that the $l_{\text {wind }}$ in these models is so large, corresponding to $f=20$ with respect to the $\mathrm{NC} r_{\text {base, }}$, that the wind mass loss is too low to remove any significant mass.
} 

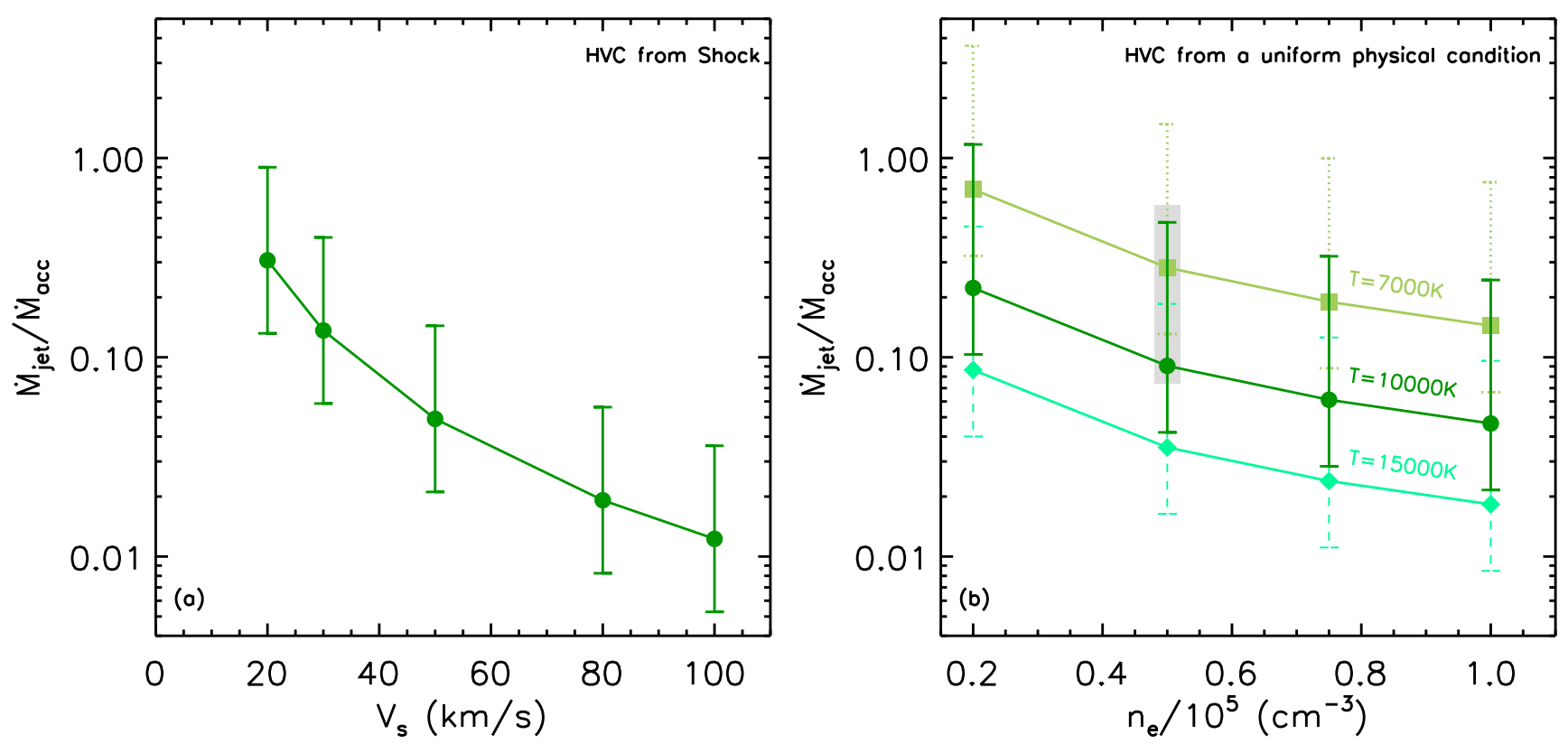

Figure 13. Left panel: median $\dot{M}_{\text {jet }} / \dot{M}_{\text {acc }}$ ratio as a function of shock velocity assuming that HVC arises in post-shock gas. Right panel: median $\dot{M}_{\text {jet }} / \dot{M}_{\text {acc }}$ as a function of electron density and for different gas temperatures assuming that HVCs are produced in uniform physical conditions. Error bars show the upper and lower quartiles of the median $\dot{M}_{\text {jet }} / \dot{M}_{\text {acc }}$ ratios for our sample, while the gray region is for sources in Nisini et al. (2018), which are reported in their paper only for $T=10,000 \mathrm{~K}$ and $n_{\mathrm{e}}=5 \times 10^{4} \mathrm{~cm}^{-3}$.

of the LVC-NC values we report here, more detailed kinematic comparisons disfavor a thermal outflow origin because several [O I] $\lambda 6300$ widths are still larger than predicted (Banzatti et al. 2018; McGinnis et al. 2018), the largest observed blueshifts occur for a lower disk inclination than predicted, and the centroids of the $\mathrm{BC}$ and $\mathrm{NC}$ are correlated (see Banzatti et al. 2018 for further details). For these reasons we favor a similar origin for both the $\mathrm{BC}$ and the $\mathrm{NC}$ in an MHD wind.

MHD wind models come in two broad categories: (1) winds arising close to the corotation radius $(\leqslant 0.1 \mathrm{au})$, such as X-winds (e.g., Shang et al. 2007), conical winds (e.g., Romanova et al. 2009), magnetospheric ejections (e.g., Zanni \& Ferreira 2009), or accretion-powered stellar winds (e.g., Matt $\&$ Pudritz 2005), and (2) winds arising over a broad range of radii in the disk, from 0.1 to $10 \mathrm{au}$, sometimes called D-winds (e.g., Pudritz et al. 2007). A key difference between these two genres of models is that the former are important in removing angular momentum from the accreting star, enabling it to spin down, and the latter in removing angular momentum from the disk itself, enabling accretion to occur through the disk rather than relying on some kind of magnetic viscosity (e.g., MRI). Note that these types of winds are not mutually exclusive. All of these scenarios can reproduce the terminal velocity of the fast $\left(\sim 100 \mathrm{~km} \mathrm{~s}^{-1}\right)$ outflowing gas traced by the HVC. However, only D-winds can account for the high angular resolution observations showing a decrease in outflow velocity with increasing jet radius, implying an "onion-like" layering of a faster flow engirdled by a slower flow (e.g., Bacciotti et al. 2000, 2002; Pyo et al. 2002, 2003; Takami et al. 2004; Beck et al. 2010; Agra-Amboage et al. 2011). Also, D-winds provide a natural explanation for possible rotation signatures in jets, implying wind footpoints spanning a large range of disk radii, from $\sim 0.1$ to 5 au (e.g., Ferreira et al. 2006; Cabrit 2009).
Our results provide strong support for the presence of D-winds, although they do not help in determining whether D-winds are the dominant contributor to the jet traced by the HVC, or whether the jet is primarily fed by an MHD wind from near the corotation radius. However, the Keplerian radii for the BC inferred from their FWHM are larger than the corotation radii $(\sim 0.05 \mathrm{au})$, and the ones for NCs are well beyond the corotation radii, implying a large range of wind radii (from $\sim 0.05$ out to $5 \mathrm{au}$ ), similar to those expected in $\mathrm{D}$-winds and inferred from jet rotation signatures. The observed correlation between the [O I] line and accretion luminosity might indicate that the wind truly drives accretion: as the [O I] $\lambda 6300$ line is optically thin, a higher luminosity implies more mass removed in the wind, and hence more angular momentum promoting mass accretion from the disk onto the central star.

Another prediction from some D-wind models is that most of the mass is lost within a few au from the central star (e.g., Pelletier \& Pudritz 1992), in agreement with our finding that the BC has larger $\dot{M}_{\text {wind }} / \dot{M}_{\text {acc }}$ than the NC. This last result also places interesting constraints on more recent disk simulations that include nonideal MHD effects (e.g., Bai 2014; Gressel et al. 2015; Béthune et al. 2017 ). These simulations show that disk winds are launched from radii extending out to $\sim 10-20$ au with vigorous mass-loss rates out to these radii (see also Bai 2017). However, even for gas at $5000 \mathrm{~K}$ we obtain an $\dot{M}_{\text {wind }} / \dot{M}_{\text {acc }}$ of 0.4 from the upper quartile of the $f=1 \mathrm{LVC}$ NC curve (Figure 12, right panel), suggesting that the massloss rate is not as large as predicted out to the $\sim 5$ au radii we can trace with the NC. If the theoretically predicted winds exist, most of the gas must be cooler $(<5000 \mathrm{~K})$ than what can be traced with the forbidden lines analyzed here. Other wind diagnostics that can probe cooler neutral hydrogen in the flow would be valuable in determining the presence of more extended disk winds. 


\section{Summary}

We analyzed optical high-resolution spectra, covering the [S II] $\lambda 4068$, [O I] $\lambda 5577$, and [O I] $\lambda 6300$ forbidden lines, from a sample of $48 \mathrm{~T}$ Tauri stars, 31 of which are surrounded by full disks and 17 by TDs. We detected the [O I] $\lambda 6300$ from 45 sources, the [O I] $\lambda 5577$ from 26 sources, and the [S II] $\lambda 4068$ from 22 sources. Following Simon et al. (2016), we decomposed the line profiles into HVC, LVC-NC, and LVCBC. As in previous studies, we attribute the line width primarily to Keplerian broadening, with forbidden emission arising in both the inner (BC within $0.5 \mathrm{au}$ ) and outer (NC within 1-5 au) disk. Many components show peak centroids blueshifted by more than $1.5 \mathrm{~km} \mathrm{~s}^{-1}$ with respect to the stellar velocity. Thus, the LVC is most likely tracing unbound slow wind gas. After flux-calibrating our spectra, we derived line luminosity and line ratios for individual kinematic components to assess the properties of the emitting gas and with the goal of measuring mass-loss rates. We also estimated mass accretion rates using 12 accretion-related permitted lines to then evaluate the mass loss over mass accretion rate. Our main results can be summarized as follows:

1. About $72 \%$ of full disks present an HVC, while this number is down to $13 \%$ for TDs. Furthermore, TDs more frequently than full disks show only an LVC-NC (44\% for TDs vs. 28\% for the full disks), while full disks tend to show line profiles with multiple components. These findings point to a depletion of gas in the inner disk of TDs, in agreement with their lower average mass accretion rate.

2. The HVC and LVC [O I] $\lambda 6300$ luminosity is confirmed to correlate with the accretion luminosity. When the [O I] $\lambda 6300 \mathrm{LVC}$ is decomposed into LVC-BC and NC, we see that the LVC-BC luminosity is more tightly correlated with accretion luminosity than the NC.

3. The profiles of individual kinematic components are similar in the [S II] $\lambda 4068$, [O I] $\lambda 5577$, and [O I] $\lambda 6300$ lines, when detected. This indicates that they trace a similar region; hence, their line ratios can be used to infer the physical properties of the emitting gas.

4. The HVC has statistically different SII40/OI63 ratios than the LVC-BC and NC. Its OI55/63 ratios are also statistically different from the LVC-BC, while they are not distinguishable from the LVC-NC. These differences and the mean line ratios corroborate previous suggestions that the $\mathrm{BC}$ traces hot/dense gas close to the base of an MHD wind.

5. Most LVC-BC and NC ratios can be explained by thermally excited gas with electron densities of $\sim 10^{7}-10^{8} \mathrm{~cm}^{-3}$ and temperatures of $5000-10,000 \mathrm{~K}$. HVC line ratios are better explained by shock models with a pre-shock number density of nucleons of $\sim 10^{6}-10^{7} \mathrm{~cm}^{-3}$.

6. Building on the [O I] $\lambda 6300 \mathrm{HVC}$ luminosity, converting a fraction of the shock mechanical energy into radiant energy, and adopting a typical shock velocity of $30 \mathrm{~km} \mathrm{~s}^{-1}$, we find a median $\dot{M}_{\text {jet }} / \dot{M}_{\text {acc }}$ ratio of 0.1 . This value is comparable to previous results from the literature that were calculated with a very different approach. The agreement between these different approaches to convert HVC luminosity into a mass outflow rate suggests that this ratio may be robust. An $\dot{M}_{\text {jet }} / \dot{M}_{\text {acc }}$ of 0.1 reflects a minimal impact on disk mass removal by outflowing material seen in the jet.

7. Building on our finding that the [O I] LVC emission is most likely thermal, gas is in LTE, and the [O I] $\lambda 6300$ line is optically thin, we estimate for the first time $\dot{M}_{\text {wind }} / \dot{M}_{\text {acc }}$ ratios separately for the $\mathrm{BC}$ and $\mathrm{NC}$. We show that $\dot{M}_{\text {wind }}$ is a factor of 5 higher in the $\mathrm{BC}$ (inner disk) than the NC (outer disk). However, absolute $\dot{M}_{\text {wind }}$ values are sensitive to the gas temperature and wind height, making direct comparisons between outflow rates in the LVC and the HVC uncertain. However, for plausible wind heights, we find that the mass flowing out in the $\mathrm{BC}$ is likely at least as large as in the HVC and may be considerably higher. If so, then the inner disk wind traced by the BC may play an important role in the evolution of the disk mass.

Taken together, our results favor D-wind models. In particular, we find that winds are launched from a range of disk radii beyond the gas corotation radius even in the LVC-BC alone. In addition, most of the mass is lost close to the star, within a few au. For plausible wind heights, LVC-NC $\dot{M}_{\text {wind }} / \dot{M}_{\text {acc }}$ ratios are lower than $\sim 0.4$, and thus lower than the ratios predicted by recent nonideal MHD simulations for radii of $\sim 1-20$ au (e.g., Bai 2017). Additional wind diagnostics tracing cooler gas would be helpful to test whether indeed radially extended winds could drive accretion.

We thank Kelle Cruz and Scott Dahm for doing the observations with Keck/HIRES. I.P., U.G., and S.E. acknowledge support from a Collaborative NSF Astronomy \& Astrophysics Research Grant (ID:1715022, ID:1713780, and ID:1714229). This material is based on work supported by the National Aeronautics and Space Administration under agreement no. NNX15AD94G for the program "Earths in Other Solar Systems." The results reported herein benefited from collaborations and/or information exchange within the NASA Nexus for Exoplanet System Science (NExSS) research coordination network sponsored by NASA's Science Mission Directorate. The data presented here were obtained at the W. M. Keck Observatory, which is operated as a scientific partnership among the California Institute of Technology, the University of California, and the National Aeronautics and Space Administration. The Observatory was made possible by the generous financial support of the W. M. Keck Foundation. The authors wish to recognize and acknowledge the very significant cultural role and reverence that the summit of Maunakea has always had within the indigenous Hawaiian community. We are most fortunate to have the opportunity to conduct observations from this mountain. This research has made use of the Keck Observatory Archive (KOA), which is operated by the W. M. Keck Observatory and the NASA Exoplanet Science Institute (NExScI), under contract with the National Aeronautics and Space Administration.

Facility: Keck:I (HIRES).

\section{Appendix A Disk Classification and $[O \mathrm{I}] \boldsymbol{\lambda} 6300$ Line Profiles}

Figures 14 and 15 show the SEDs of our sources. SEDs are constructed using the Two Micron All Sky Survey (2MASS; 


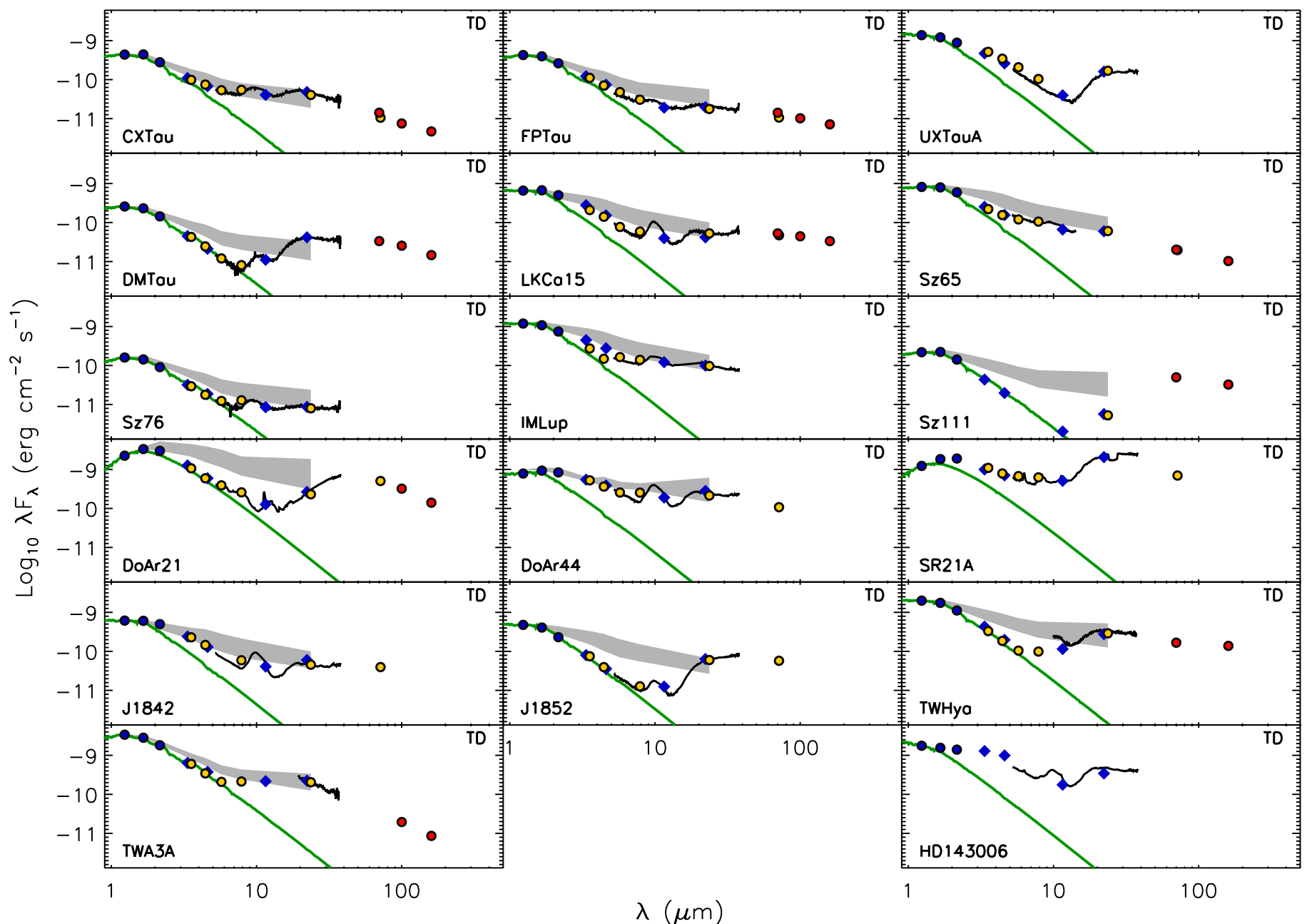

Figure 14. SEDs for the TDs analyzed in this paper. Filled circles are photometric data from 2MASS (blue), Spitzer (yellow), and Herschel (red). Open circles are used for WISE photometry. The black solid line shows the Spitzer IRS spectrum, while the green solid line shows the stellar photosphere. The gray region shows the upper and lower quartiles of the Lynds 1641 CTTS median SED. The median SED has been reddened with the extinction of each source and then normalized to the $J$ band flux.

Skrutskie et al. 2006), the Spitzer and Herschel photometric data, the Spitzer IRS spectra, and the Wide-field Infrared Survey Explorer (WISE; Wright et al. 2010). For the sources in Taurus, their Spitzer data are collected from Luhman et al. (2010) and Rebull et al. (2010), and the Herschel data are from Howard et al. (2013). For the sources in Lupus and $\rho$ Oph, the Spitzer data are from the c2d catalog (Evans et al. 2003), and the Herschel data are from Bustamante et al. (2015) and Rebollido et al. (2015). For the sources in Corona Australis, the Spitzer data are collected from Hillenbrand et al. (2008) and Dunham et al. (2015), and the Herschel data are from Sicilia-Aguilar et al. (2013). For the two sources in the TW Hya association, their Spitzer data are collected from Luhman et al. (2010), and their Herschel data are from Riviere-Marichalar et al. (2013).

For each source in our sample, we construct a median SED from CTTSs with similar spectral type in Lynds 1641
(Fang et al. 2009, 2013a). The age of Lynds 1641 is around 1-2 Myr (Fang et al. 2013a), which is similar to most of the sources in this work, besides the TW Hya association. In each panel of Figures 14 and 15, we show in gray the upper and lower quartiles of the median SEDs. We classify a disk as TD if its SED is below the lower quartile of the Lynds 1641 median SED at more than two bands, and otherwise as a full disk. As Lynds 1641 lacks G- and F-type stars (Fang et al. 2013a), we cannot use our approach to classify SR 21A, HD 143006, V1057 Cyg, V1515 Cyg, and DI Cep. In this case we adopt the TD classification for SR 21A and HD 143006, found in the literature (Najita et al. 2015; van der Marel et al. 2016), and consider the other three ones as full disks.

Figures 16 and 17 show the [O I] $\lambda 6300$ line profiles for the TDs and full disks. It is clear that the TDs have simpler forbidden-line profiles than the full disks. 


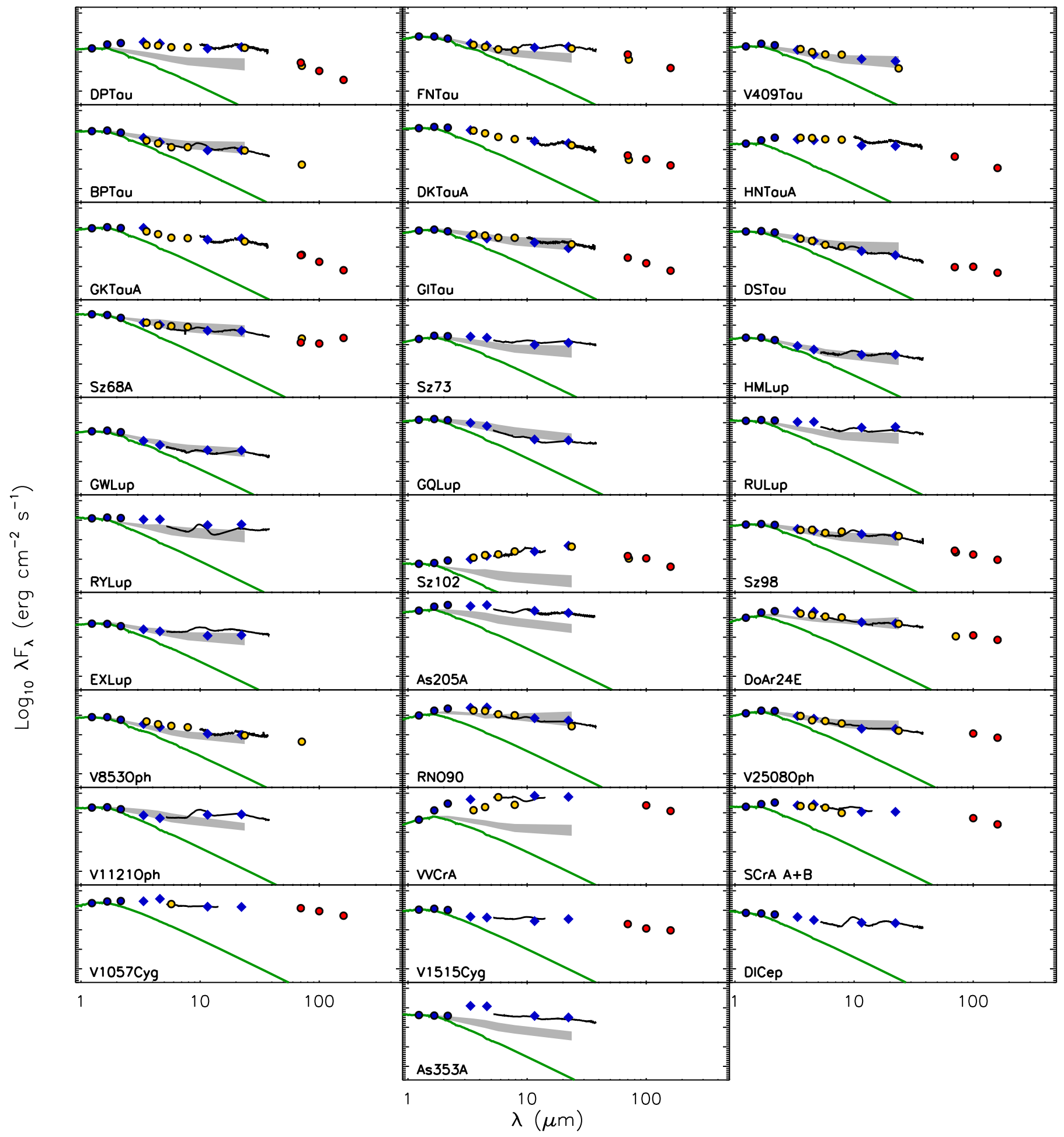

Figure 15. Same as Figure 14, but for full disks. 


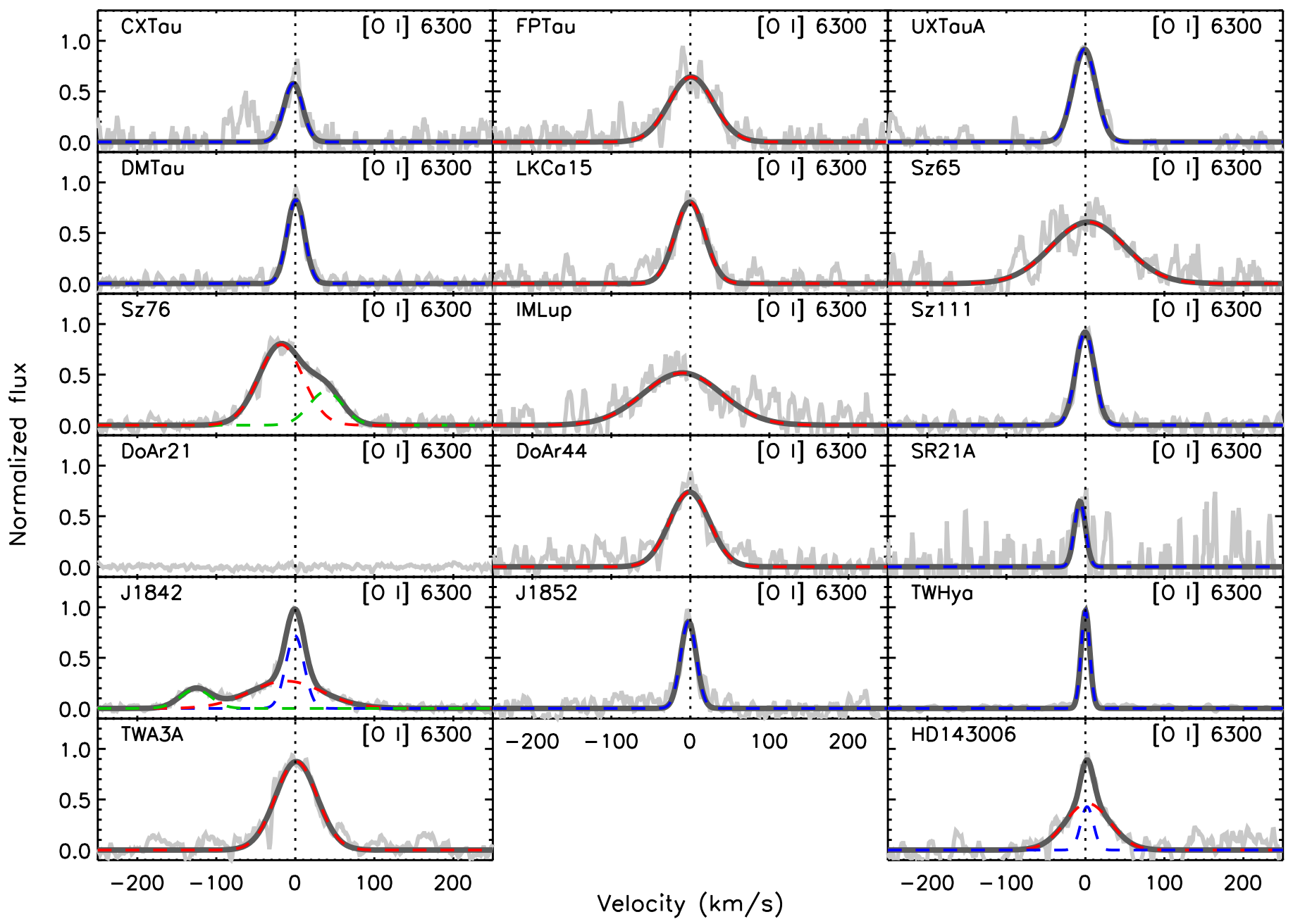

Figure 16. [O I] $\lambda 6300$ line profiles for TDs. The SEDs of the corresponding sources are shown in Figure 14. 


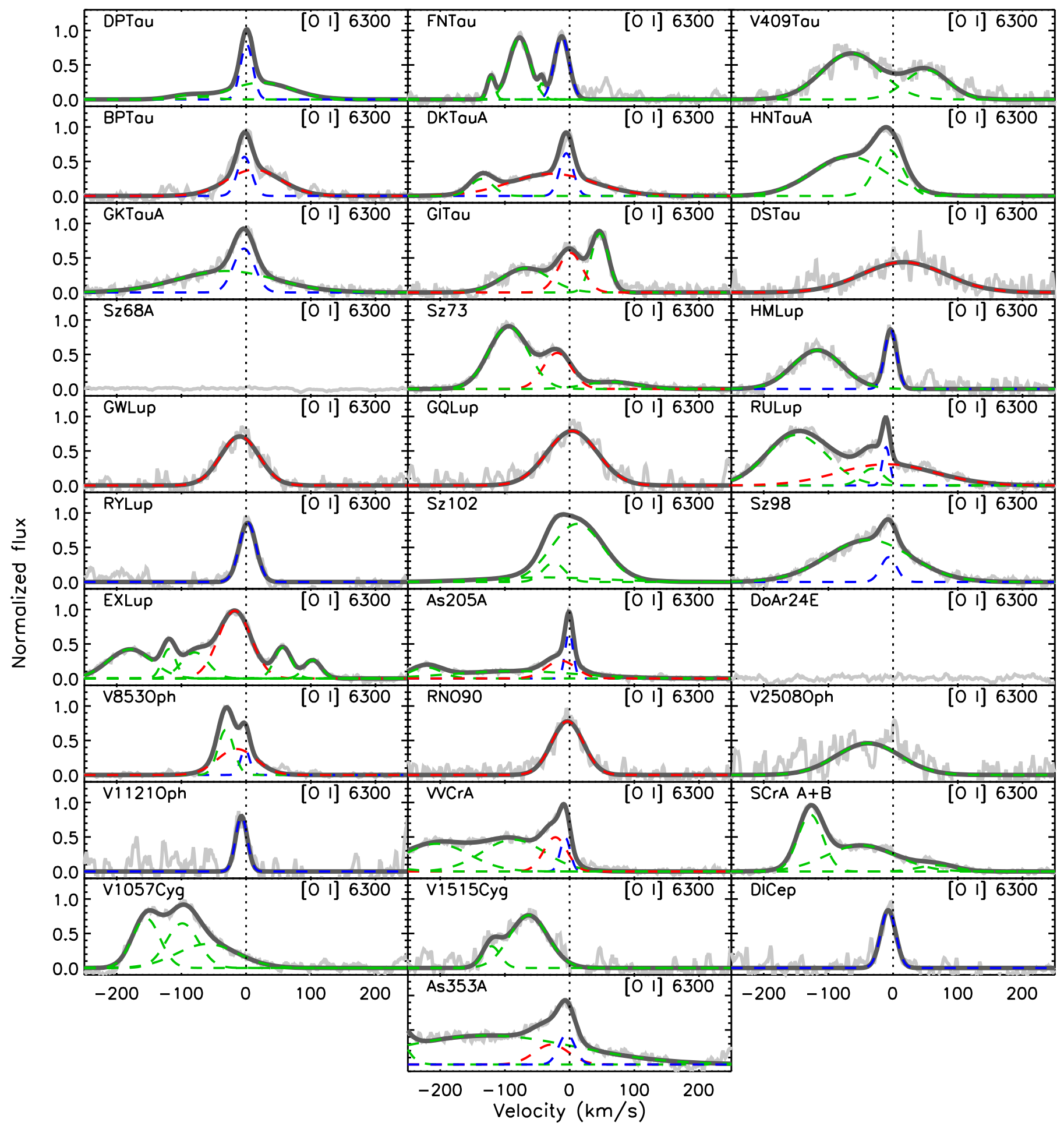

Figure 17. [O I] $\lambda 6300$ line profiles for full disks. The SEDs of the corresponding sources are shown in Figure 15.

\section{Appendix B \\ Flux Calibration}

We use three spectrophotometric standards each night to flux-calibrate the spectra of our scientific targets. First, we obtain the BT-settl model atmosphere (Allard et al. 2011) corresponding to each telluric standard spectral type in the night of 2008 January 23 and the white dwarf model atmosphere (Koester 2010) for the white dwarf telluric standards in the night of 2008 May 23. Next, we fit its broadband photometry using the aforementioned model with two free parameters, extinction and stellar angular radius, as in Fang et al. (2009, 2013a). Then, we shift and rotationally broaden the best-fit model atmosphere and degrade it to the Keck spectral resolution. In each order, the ratio between the observed and model spectrum is fitted with a fourth-order polynomial function, from which we obtain the conversion from counts to absolute flux. The spectrophotometric standard that is observed closest in time to each of our targets is chosen 


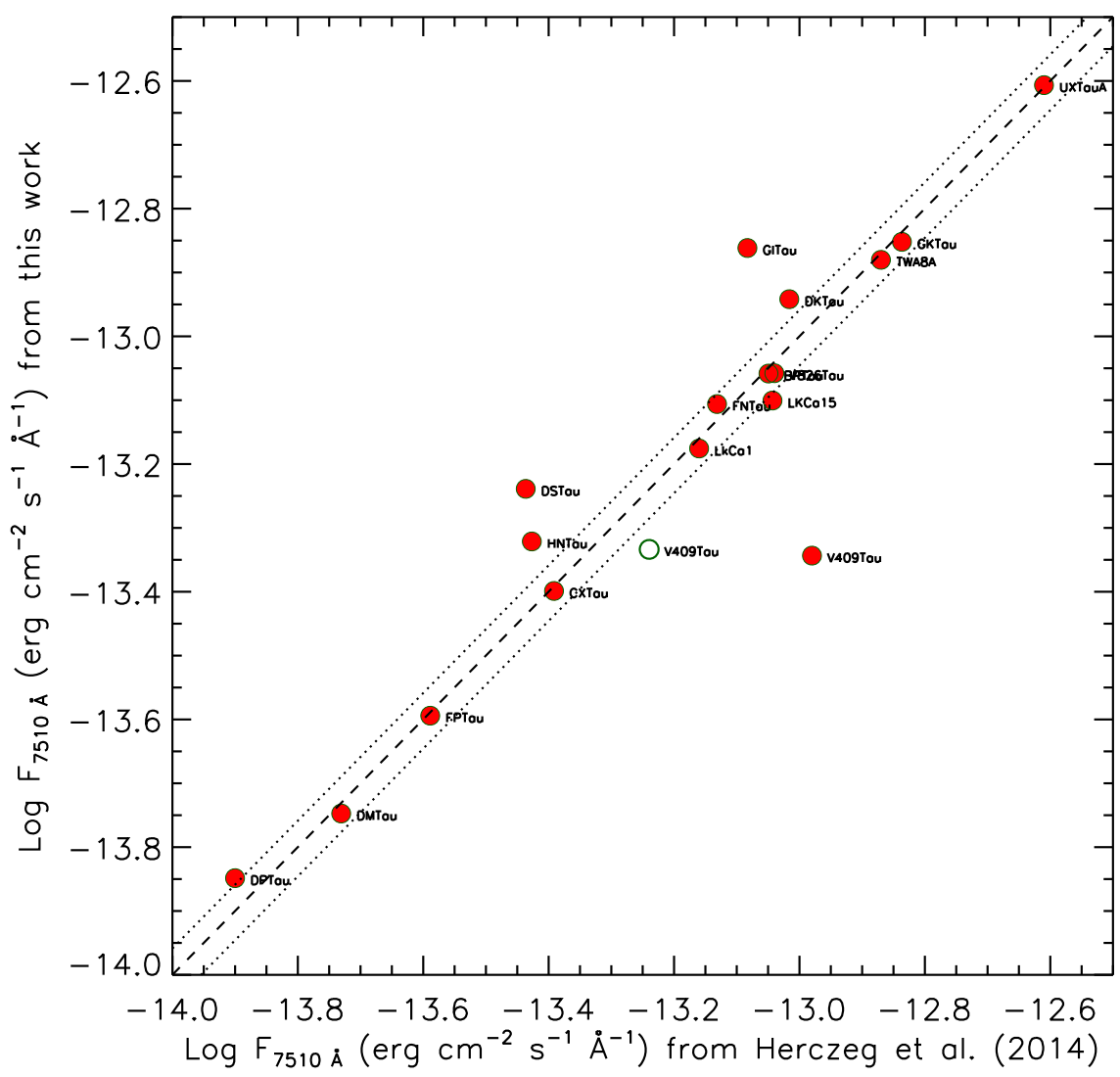

Figure 18. Comparison of $7510 \AA$ fluxes from our work and from Herczeg \& Hillenbrand (2014). The open circle also locates our $7510 \AA$ flux for V409 Tau with respect to the SDSS photometry. The dashed line shows the 1:1 line, while dotted lines mark a \pm 0.04 dex difference.

as its flux calibration. As our slit width is narrow (0". 861), we also correct for the missing flux due to the difference in point spread functions between the target and the spectrophotometric standard. Atmospheric absorption due to the different air mass between the spectrophotometric standards and the scientific objects is also corrected using optical extinction coefficients proper for Maunakea. ${ }^{21}$ The flux calibration did not account for atmospheric dispersion in the observations of DK Tau A, where the slit position angle was offset from the parallactic angle. For SCrA A + B, the MAKEE pipeline cannot resolve the SCrA A and B components, and thus the flux calibration overcorrects the flux for the slit loss by $\sim 20 \%$.

We use two methods to estimate the uncertainty on the flux calibration. The first one is to flux-calibrate one spectrophotometric standard using another close-in-time spectrophotometric standard and compare the calibrated flux with the expected one. We found differences of $\sim 14 \%$ for the night on 2008 January 23 and only 3\% for the night on 2008 May 23. To evaluate the uncertainty on line ratios close to the forbidden lines analyzed here, we also calculate the $F_{\text {cont }, 4068} / F_{\text {cont,6300 }}$ and the $F_{\text {cont,5577 }} / F_{\text {cont,6300, basically }}$ continuum fluxes near the $[\mathrm{S} \mathrm{II}] \lambda 4068$, [O I] $\lambda 5577$, and [O I] $\lambda 6300$ transitions, from the flux-calibrated spectra of one spectrophotometric standard using another one close in time. When comparing them with the expected values, we find differences of $\sim 5 \%$ and $3 \%$ on 2008 January 23 and $\sim 5 \%$

\footnotetext{
$\overline{21}$ The coefficients used are listed at www.gemini.edu/sciops/telescopes-andsites/observing-condition-constraints/extinction.
}

and $1 \%$ on 2008 May 23 for the $F_{\text {cont,4068 }} / F_{\text {cont,6300 }}$ and $F_{\text {cont,5577 }} / F_{\text {cont,6300 }}$ ratios, respectively.

The data acquired on 2008 January 23 cover wavelengths near $7510 \AA$ A Hence, we can further estimate the uncertainty on our flux calibration by comparing our calibrated $7510 \AA$ fluxes with those reported in Herczeg \& Hillenbrand (2014). Unfortunately, the $7510 \AA$ wavelength is located in the gap between two HIRES orders. So, we did not measure the $7510 \AA$ flux directly, but instead interpolate the flux using wavelengths near $7510 \AA$. Figure 18 shows our flux values compared to those from Herczeg \& Hillenbrand (2014). The mean difference between the two works is about 0.07 dex. Four sources, DS Tau, HN Tau A, GI Tau, and V409 Tau, show larger differences $(0.10-0.37 \mathrm{dex})$, which may be due to their brightness variability (see Rodriguez et al. 2015 for V409 Tau and Guo et al. 2018 for GI Tau). V409 Tau, which sports the largest difference $(0.37 \mathrm{dex})$, has been also observed with the Sloan Digital Sky Survey (SDSS; Ahn et al. 2012) and is $11.934 \mathrm{mag}$ in SDSS $-i$ band. We convert the $i$-band photometry to flux and correct for the extinction $A_{\mathrm{V}}=1$ from Herczeg \& Hillenbrand (2014), which gives a flux of $5.76 \times 10^{-14} \mathrm{erg} \mathrm{s}^{-1} \mathrm{~cm}^{-2} \AA^{-1}$ at $7625 \AA$ (the center of SDSS $-i$ band). The difference between our $7510 \AA$ flux and the SDSS $7625 \AA$ flux is $\sim 0.09 \mathrm{dex}$, much lower than that with Herczeg \& Hillenbrand (2014), which supports the hypothesis of variability driving a larger difference. If we exclude the four outliers in Figure 18, the mean difference between our $7510 \AA$ fluxes and those from Herczeg \& Hillenbrand (2014) is reduced to $\sim 0.03$ dex. In summary, two different methods suggest that 
the uncertainty on our absolute flux calibration is likely better than $10 \%$.

\section{Appendix C \\ Comments on Sources without $\left[\begin{array}{l}\mathrm{O} \\ \mathbf{1}\end{array} \mathbf{\lambda 6 3 0 0}\right.$ Detection}

Our sample includes three sources (SZ 68A, DoAr 21, and DoAr 24E) with no [O I] $\lambda 6300$ detection. Neither DoAr 21 nor DoAr 24E shows emission lines in our spectra that can be used to derive accretion luminosities; thus, they are inferred to have weak or absent disk accretion. In the following, we provide additional information on these sources.

SZ 68A is a a triple system with a close binary (separation 0 ". 126) and a third faint companion (M6) with a separation of 2".808 (Correia et al. 2006). Our spectroscopic observation targeted the close binary. We detect the Ca II lines at 3934 and $3968 \AA$ but only weak $\mathrm{H} \alpha$ emission $(\mathrm{EW} \sim-2.8 \AA$ ). This star has been classified as a weak-line $\mathrm{T}$ Tauri star, a young nonaccreting star, by Cieza et al. (2007) and Alcalá et al. (2017). The [O I] $\lambda 6300$ emission is not detected in our HIRES spectra, and it is also absent in the X-Shooter spectra by Nisini et al. (2018). Ansdell et al. (2016) reported cold CO gas emission around the binary and no gas or dust cavity at a resolution of $50 \times 40 \mathrm{au}$. The SED of SZ 68A is compatible with that of a full disk.

DoAr 21 is a close binary with a separation of 1.2-1.8 au (Loinard et al. 2008). The HIRES spectrum of DoAr 21, which covers from 4420 to $6310 \AA$, does not show any accretionrelated emission lines. Salyk et al. (2013) detected $\operatorname{Pf} \beta$ emission from DoAr 21 and estimated a mass accretion rate of $1.7 \times 10^{-8} M_{\odot} \mathrm{yr}^{-1}$. DoAr 21 is detected at far-infrared wavelengths (Rebollido et al. 2015) and has reduced midinfrared emission as TDs (see Figure 14 from our paper).
However, based on high spatial resolution mid-infrared imagery, Jensen et al. (2009) find very weak, almost absent, excess emission from this source at $9-18 \mu \mathrm{m}$ and argue that low-resolution data could be contaminated by an extended infrared source. Thus, they suggested that either the disk is in the final stage of clearing or there is no disk around DoAr 21. The recent ALMA imaging of DoAr 21 at $870 \mu \mathrm{m}$ shows no detection at a spatial resolution of 0.2 and a sensitivity of $0.02 \mathrm{mJy}$ (Cox et al. 2017). Therefore, even if there is a disk around DoAr 21, its disk mass should be very low.

DoAr 24E has a faint companion at a separation of 2."03 (Ratzka et al. 2005). Our HIRES spectrum covering from 4420 to $6310 \AA$ does not show any accretion-related emission lines. Natta et al. (2006) detected $\mathrm{Pa} \beta$ emission from its near-infrared spectrum and estimated a mass accretion rate of $6.2 \times 10^{-9} M_{\odot} \mathrm{yr}^{-1}$. The source SED is compatible with that of full disks. The continuum emission from the disk has been detected at far-infrared, submillimeter, and millimeter wavelengths (Mohanty et al. 2013; Rebollido et al. 2015). No [O I] and $\mathrm{o}-\mathrm{H}_{2} \mathrm{O}$ emission is detected around $63 \mu \mathrm{m}$ (RiviereMarichalar et al. 2016).

\section{Appendix D \\ Corrected Line Profiles and Kinematic Decomposition}

The rationale for decomposing corrected line profiles with Gaussians is discussed in Section 3.2, while the best-fit parameters are summarized in Table 2. Figure 19 provides the best-fit Gaussian profiles color-coded by kinematic component and superimposed on the corrected [S II] $\lambda 4068$, $[\mathrm{OI}] \lambda 5577$, and [O I] $\lambda 6300$ profiles for all the sources in our sample. 


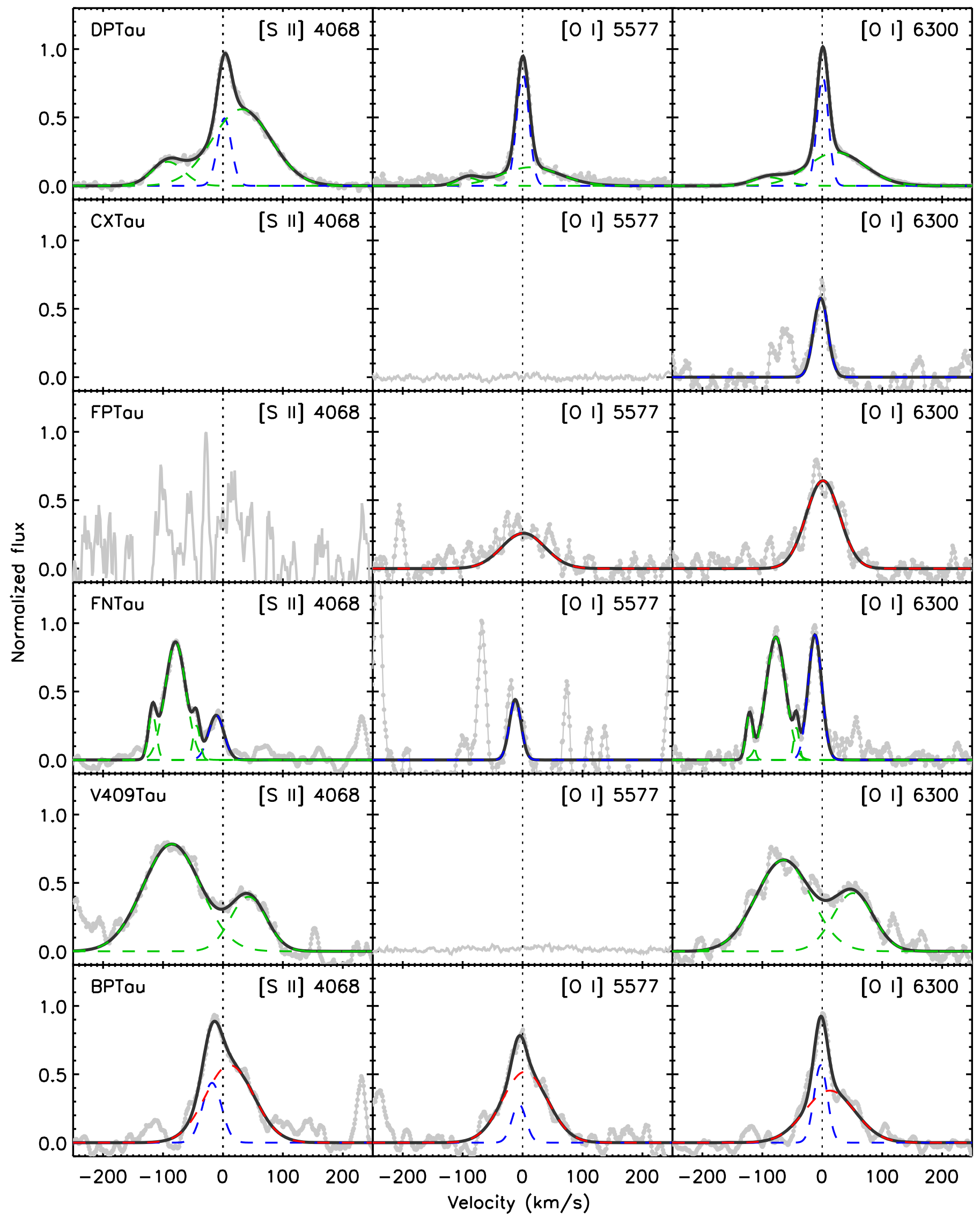

Figure 19. [S II] $\lambda 4068$, [O I] $\lambda 6300$, and [O I] $\lambda 5577$ line profiles for our sample. In each panel, the green dashed line is for the HVC, the red dashed line is for the LVC-BC, the blue dashed line is for the LVC-NC, and the dark solid line is the sum of all components. For EX Lup, Fe lines contaminating the profiles of the forbidden lines are marked with vertical dashed light-blue lines. (An extended version of this figure is available.) 


\section{Appendix E}

\section{Line Decontamination}

Seven sources present [S II] $\lambda 4068$ line profiles that are highly contaminated by Fe I emission at 4063.6 and $4071.7 \AA$. In addition, the [OI] $\lambda 5577$ line from RU Lup is also contaminated by Fe I emission at $5572.8 \AA$ A. Figure 20 shows the contaminated line profiles. We fit the wings of the Fe I emission with linear functions and tentatively decontaminate the profiles by subtracting the fitted function from the observed spectra. The corrected [S II] $\lambda 4068$ and [O I] $\lambda 5577$ line profiles are also shown in Figure 20. Due to the strong contamination from the $\mathrm{Fe}$ I line at $4063.6 \AA$, any [S II] $\lambda 4068$ flux more blueshifted than $\sim 200 \mathrm{~km} \mathrm{~s}^{-1}$ cannot be recovered. A comparison of [S II] $\lambda 4068$ and [O I] $\lambda 6300$ profiles suggests that we achieve a good correction for less blueshifted emission (see Figure 5 in the main text).
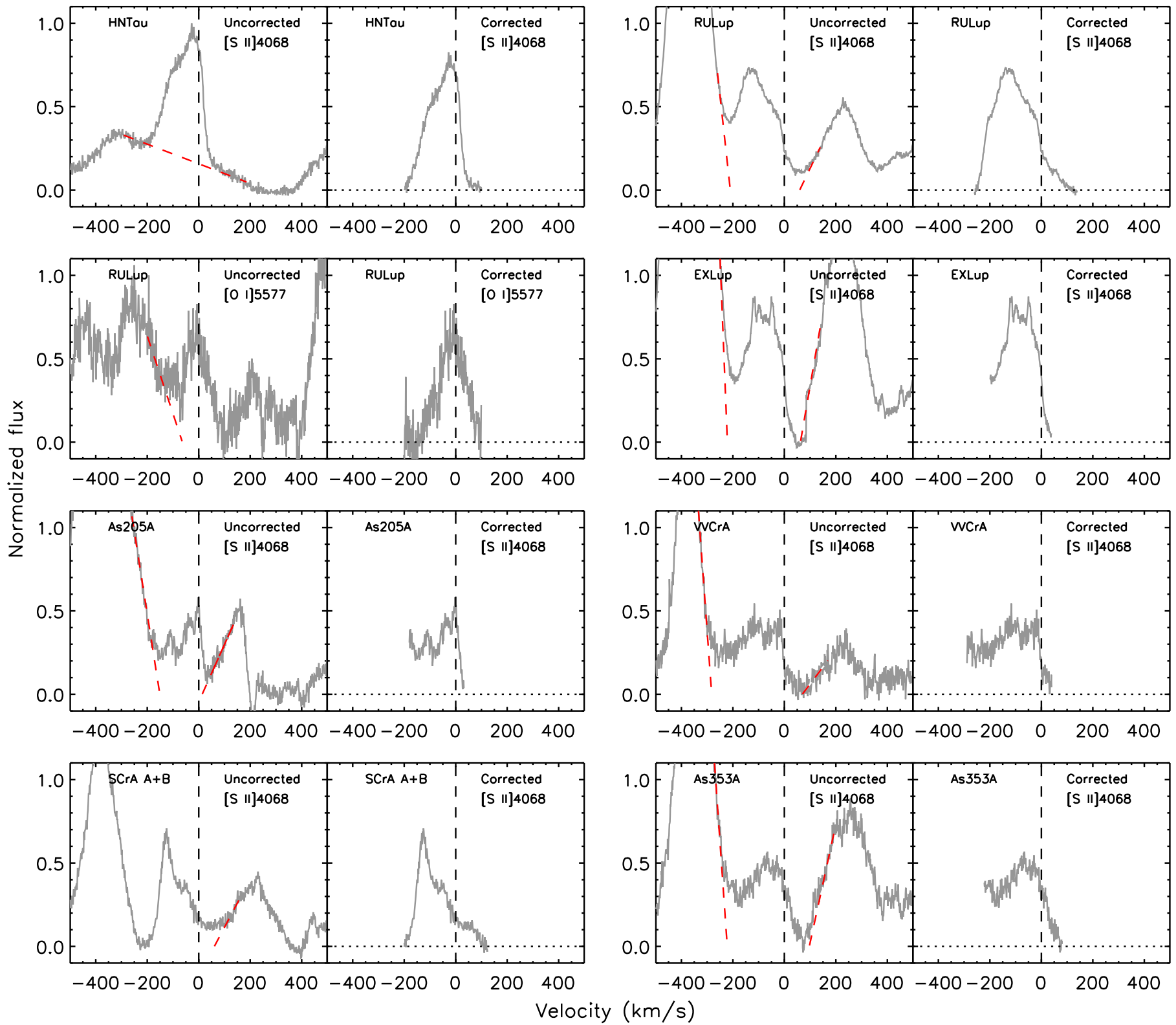

Figure 20. Profiles that are contaminated by Fe I lines (left panels). The right panels show our corrected profiles. 


\section{Appendix F \\ A Comparison of the Individual Components in [S II] $\lambda 4068$ and $\left[\begin{array}{ll}\mathrm{I}\end{array}\right] \boldsymbol{2} \mathbf{6 3 0 0}$}

As discussed in the main text (Section 4.1), individual kinematic components present similar [S II] $\lambda 4068$, [O I] $\lambda 5577$, and $[\mathrm{OI}] \lambda 6300$ profiles, indicating that they trace the same physical region. Here, we provide further evidence to this finding by showing a comparison of centroids and FWHMs of individual [S II] $\lambda 4068$ and $[\mathrm{O} \mathrm{I}] \lambda 5577$ components versus the [OI] $\lambda 6300$ components (see Figures 21 and 22). Note the similarity in centroids and FWHMs for individual kinematic components.
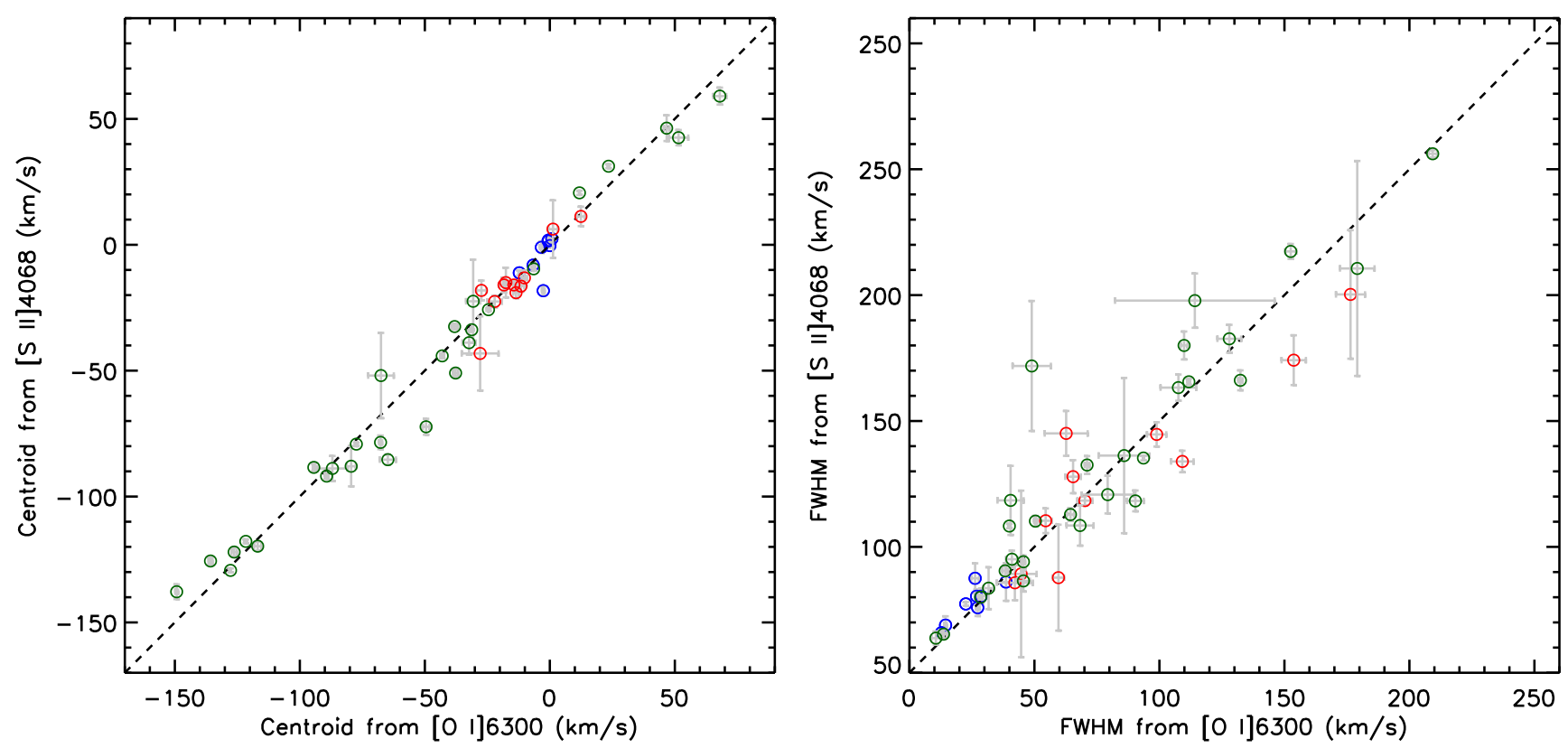

Figure 21. Centroids (left) and FWHMs (right) of individual [S II] $\lambda 4068$ and [O I] $\lambda 6300$ components (HVC in green, LVC-BC in red, and LVC-NC in blue). A dashed line shows the 1:1 relation.
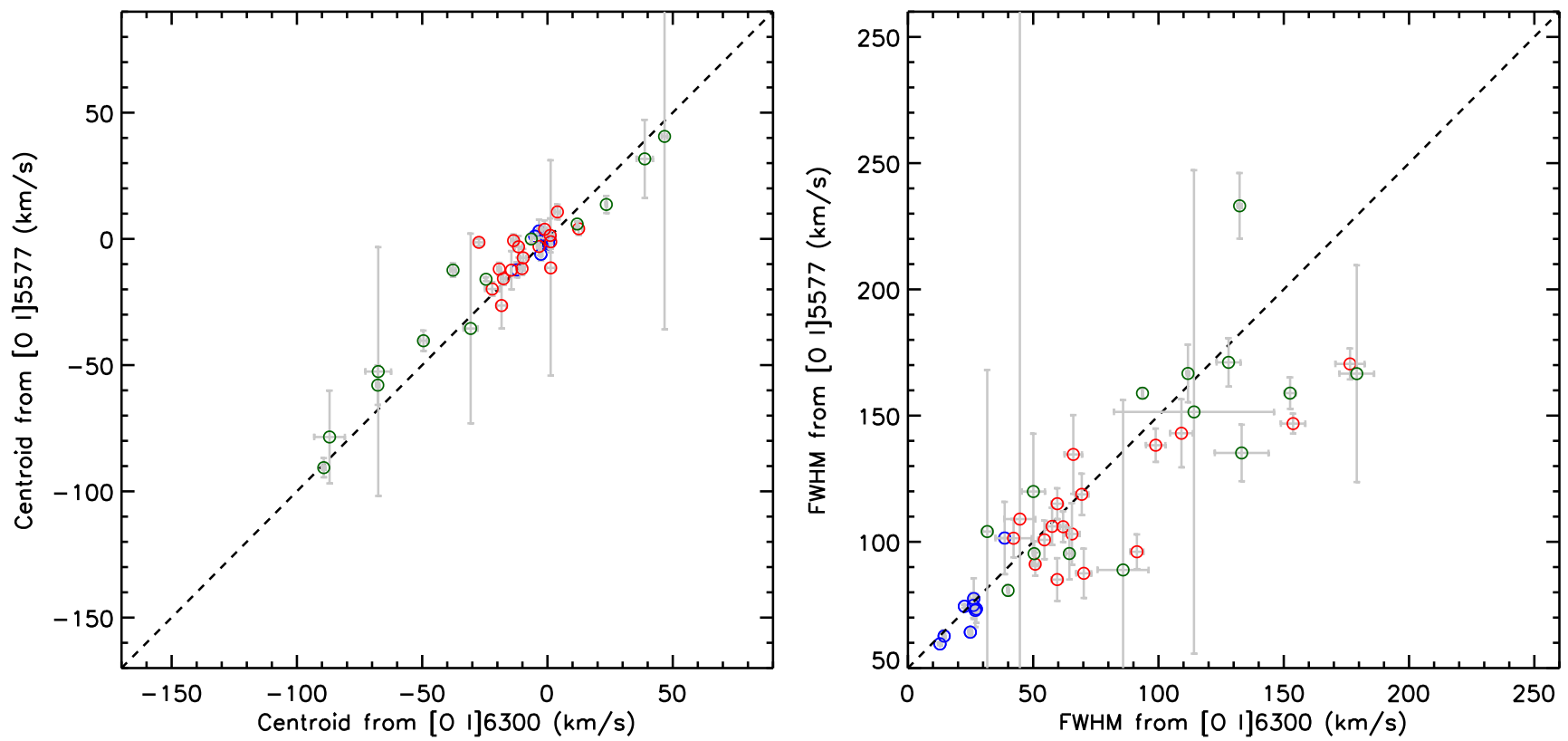

Figure 22. Same as Figure 21, but for the [O I] $\lambda 5577$ and [O I] $\lambda 6300$ lines. 


\section{Appendix G \\ Updated Relation between Line and Accretion Luminosity}

In view of the Gaia DR2 release (Bailer-Jones et al. 2018), we revisit the relations between the luminosity of permitted lines $\left(L_{\text {line }}\right)$ and the accretion luminosity $\left(L_{\text {acc }}\right)$. We collect $L_{\text {line }}$ and $L_{\text {acc }}$ from Alcalá et al. (2017) and scale them to the new Gaia DR2 distance of each source. ${ }^{22}$ We carry out a linear regression to fit $\log L_{\text {acc }}=a \times \log L_{\text {line }}+b$. We have excluded weak (or dubious) accretors and subluminous objects from the fit. The best-fit $a$ and $b$ coefficients and their uncertainties are listed in Table 7. Within $1 \sigma$, most of them are the same as those reported in Alcalá et al. (2017).

Table 7

Revised $\log L_{\text {acc }}-\log L_{\text {line }}$ Linear Fit

\begin{tabular}{lccc}
\hline \hline Lines & $\lambda(\AA)$ & $a( \pm$ err $)$ & $b( \pm$ err $)$ \\
\hline $\mathrm{H} \alpha$ & 6562.80 & $1.15( \pm 0.05)$ & $1.81( \pm 0.20)$ \\
$\mathrm{H} \beta$ & 4861.33 & $1.15( \pm 0.03)$ & $2.64( \pm 0.16)$ \\
$\mathrm{H} \gamma$ & 4340.46 & $1.12( \pm 0.03)$ & $2.75( \pm 0.17)$ \\
$\mathrm{H} \delta$ & 4101.73 & $1.09( \pm 0.04)$ & $2.74( \pm 0.19)$ \\
$\mathrm{H} \zeta$ & 3889.05 & $1.06( \pm 0.03)$ & $2.76( \pm 0.18)$ \\
\hline $\mathrm{Pa} \beta$ & 12818.07 & $1.08( \pm 0.07)$ & $2.81( \pm 0.36)$ \\
$\mathrm{Pa} \gamma$ & 10938.09 & $1.21( \pm 0.06)$ & $3.43( \pm 0.29)$ \\
$\mathrm{Pa} \delta$ & 10049.37 & $1.27( \pm 0.09)$ & $3.93( \pm 0.44)$ \\
\hline $\mathrm{Br} \gamma$ & 21661.2 & $1.20( \pm 0.11)$ & $4.07( \pm 0.57)$ \\
\hline $\mathrm{He}$ I & 4026.19 & $1.05( \pm 0.04)$ & $3.67( \pm 0.22)$ \\
$\mathrm{He}$ I & 4471.48 & $1.05( \pm 0.04)$ & $3.48( \pm 0.23)$ \\
$\mathrm{He}$ I & 4713.15 & $0.86( \pm 0.08)$ & $3.02( \pm 0.50)$ \\
$\mathrm{He}$ I & 5015.68 & $1.01( \pm 0.04)$ & $3.61( \pm 0.24)$ \\
$\mathrm{He}$ I & 5875.62 & $1.17( \pm 0.04)$ & $3.78( \pm 0.22)$ \\
$\mathrm{He}$ I & 6678.15 & $1.24( \pm 0.06)$ & $4.71( \pm 0.34)$ \\
$\mathrm{He}$ I & 7065.19 & $1.20( \pm 0.05)$ & $4.57( \pm 0.29)$ \\
$\mathrm{He}$ II & 4685.80 & $1.05( \pm 0.05)$ & $3.90( \pm 0.33)$ \\
\hline $\mathrm{Ca}$ II $(\mathrm{K})$ & 3933.66 & $1.05( \pm 0.04)$ & $2.60( \pm 0.18)$ \\
$\mathrm{Ca}$ II $(\mathrm{H})$ & 3968.47 & $1.09( \pm 0.03)$ & $2.78( \pm 0.16)$ \\
\hline
\end{tabular}

\section{ORCID iDs}

Min Fang (다 https://orcid.org/0000-0001-8060-1321 Ilaria Pascucci (i) https://orcid.org/0000-0001-7962-1683 Uma Gorti (1) https://orcid.org/0000-0002-3311-5918 Andrea Banzatti (i) https://orcid.org/0000-0003-4335-0900 Mario Flock (1) https://orcid.org/0000-0002-9298-3029 Patrick Hartigan (1) https://orcid.org/0000-0002-5380-549X Gregory J. Herczeg (1) https://orcid.org/0000-0002-7154-6065 Andrea K. Dupree (i) https://orcid.org/0000-0002-8985-8489

\section{References}

Agra-Amboage, V., Dougados, C., Cabrit, S., \& Reunanen, J. 2011, A\&A, 532, A59

Ahn, C. P., Alexandroff, R., Allende Prieto, C., et al. 2012, ApJS, 203, 21 Alcalá, J. M., Manara, C. F., Natta, A., et al. 2017, A\&A, 600, A20 Alcalá, J. M., Natta, A., Manara, C. F., et al. 2014, A\&A, 561, A2 Alexander, R. D. 2008, MNRAS, 391, L64

\footnotetext{
$\overline{22}$ Note that Alcalá et al. (2017) take 200 pc as the distance of Lupus III However, the Gaia DR2 distance of most sources in Lupus III is $\sim 160$ pc.
}

Allard, F., Homeier, D., \& Freytag, B. 2011, in ASP Conf. Ser. 448, 16th Cambridge Workshop on Cool Stars, Stellar Systems, and the Sun, ed. C. Johns-Krull, M. K. Browning, \& A. A. West (San Francisco, CA: ASP), 91

Andrews, S. M., Wilner, D. J., Hughes, A. M., Qi, C., \& Dullemond, C. P. 2010, ApJ, 723, 1241

Ansdell, M., Williams, J. P., van der Marel, N., et al. 2016, ApJ, 828, 46

Armitage, P. J., Simon, J. B., \& Martin, R. G. 2013, ApJL, 778, L14

Asplund, M., Grevesse, N., \& Sauval, A. J. 2005, in ASP Conf. Ser. 336, Cosmic Abundances as Records of Stellar Evolution and Nucleosynthesis, ed. T. G. Barnes, III \& F. N. Bash (San Francisco, CA: ASP), 25

Audard, M., Ábrahám, P., Dunham, M. M., et al. 2014, in Protostars and Planets VI, ed. H. Beuther et al. (Tucson, AZ: Univ. Arizona Press), 387

Bacciotti, F., Mundt, R., Ray, T. P., et al. 2000, ApJL, 537, L49

Bacciotti, F., Ray, T. P., Mundt, R., Eislöffel, J., \& Solf, J. 2002, ApJ, 576, 222 Bai, X.-N. 2014, ApJ, 791, 137

Bai, X.-N. 2017, ApJ, 845, 75

Bai, X.-N., \& Stone, J. M. 2013, ApJ, 769, 76

Bai, X.-N., \& Stone, J. M. 2017, ApJ, 836, 46

Bailer-Jones, C. A. L., Rybizki, J., Fouesneau, M., Mantelet, G., \& Andrae, R. 2018, AJ, 156, 58

Banzatti, A., Pascucci, I., Edwards, S., et al. 2018, ApJ, submitted

Beck, T. L., Bary, J. S., \& McGregor, P. J. 2010, ApJ, 722, 1360

Bertaux, J. L., Lallement, R., Ferron, S., Boonne, C., \& Bodichon, R. 2014, A\&A, 564, A46

Béthune, W., Lesur, G., \& Ferreira, J. 2017, A\&A, 600, A75

Blandford, R. D., \& Payne, D. G. 1982, MNRAS, 199, 883

Bustamante, I., Merín, B., Ribas, Á., et al. 2015, A\&A, 578, A23

Cabrit, S. 2009, ASSP, 13, 247

Cieza, L., Padgett, D. L., Stapelfeldt, K. R., et al. 2007, ApJ, 667, 308

Cohen, M., \& Kuhi, L. V. 1979, ApJS, 41, 743

Correia, S., Zinnecker, H., Ratzka, T., \& Sterzik, M. F. 2006, A\&A, 459, 909

Cox, E. G., Harris, R. J., Looney, L. W., et al. 2017, ApJ, 851, 83

Dere, K. P., Landi, E., Mason, H. E., Monsignori Fossi, B. C., \& Young, P. R. 1997, A\&AS, 125, 149

Donaldson, J. K., Weinberger, A. J., Gagné, J., et al. 2016, ApJ, 833, 95

Dougados, C., Bacciotti, F., Cabrit, S., \& Nisini, B. 2010, in Jets from Young Stars IV, Vol. 793, ed. P. J. V. Garcia \& J. M. Ferreira (Berlin: Springer), 213

Dunham, M. M., Allen, L. E., Evans, N. J., II, et al. 2015, ApJS, 220, 11

Edwards, S., Cabrit, S., Strom, S. E., et al. 1987, ApJ, 321, 473

Ercolano, B., \& Owen, J. E. 2016, MNRAS, 460, 3472

Ercolano, B., \& Pascucci, I. 2017, RSOS, 4, 170114

Espaillat, C., Muzerolle, J., Najita, J., et al. 2014, in Protostars and Planets VI, ed. H. Beuther et al. (Tucson, AZ: Univ. Arizona Press), 497

Evans, N. J., II, Allen, L. E., Blake, G. A., et al. 2003, PASP, 115, 965

Fang, M., Kim, J. S., van Boekel, R., et al. 2013a, ApJS, 207, 5

Fang, M., van Boekel, R., Bouwman, J., et al. 2013b, A\&A, 549, A15

Fang, M., van Boekel, R., Wang, W., et al. 2009, A\&A, 504, 461

Fang, Q., Herczeg, G. J., \& Rizzuto, A. 2017, ApJ, 842, 123

Feiden, G. A. 2016, A\&A, 593, A99

Feigelson, E. D., \& Nelson, P. I. 1985, ApJ, 293, 192

Ferreira, J., Dougados, C., \& Cabrit, S. 2006, A\&A, 453, 785

Frank, A., Ray, T. P., Cabrit, S., et al. 2014, in Protostars and Planets VI, ed. H. Beuther et al. (Tucson, AZ: Univ. Arizona Press), 451

Frasca, A., Biazzo, K., Alcalà, J. M., et al. 2017, A\&A, 602, A33

Fromang, S., Latter, H., Lesur, G., \& Ogilvie, G. I. 2013, A\&A, 552, A71

Gammie, C. F. 1996, ApJ, 457, 355

Giannini, T., Antoniucci, S., Nisini, B., Bacciotti, F., \& Podio, L. 2015, ApJ, 814,52

Giannini, T., Nisini, B., Antoniucci, S., et al. 2013, ApJ, 778, 71

Gorti, U., Hollenbach, D., Najita, J., \& Pascucci, I. 2011, ApJ, 735, 90

Gorti, U., Liseau, R., Sándor, Z., \& Clarke, C. 2016, SSRv, 205, 125

Green, J. D., Hartmann, L., Calvet, N., et al. 2006, ApJ, 648, 1099

Gressel, O., Turner, N. J., Nelson, R. P., \& McNally, C. P. 2015, ApJ, 801,84

Guilloteau, S., Dutrey, A., Piétu, V., \& Boehler, Y. 2011, A\&A, 529, A105

Gullbring, E., Hartmann, L., Briceno, C., \& Calvet, N. 1998, ApJ, 492, 323

Guo, Z., Herczeg, G. J., Jose, J., et al. 2018, ApJ, 852, 56

Hales, A. S., Peréz, S., Saito, M., et al. 2018, ApJ, 859, 111

Harich, S. A., Hwang, D. W. H., Yang, X., et al. 2000, JChPh, 113, 10073

Hartigan, P., Edwards, S., \& Ghandour, L. 1995, ApJ, 452, 736

Hartigan, P., Hartmann, L., Kenyon, S., Hewett, R., \& Stauffer, J. 1989, ApJS, 70,899

Hartigan, P., \& Morse, J. 2007, ApJ, 660, 426

Hartigan, P., Morse, J. A., \& Raymond, J. 1994, ApJ, 436, 125

Hartigan, P., Raymond, J., \& Hartmann, L. 1987, ApJ, 316, 323

Hartigan, P., \& Wright, A. 2015, ApJ, 811, 12 
Herbig, G. H. 1977, ApJ, 217, 693

Herbig, G. H., \& Bell, K. R. 1988, Third Catalog of Emission-Line Stars of the Orion Population, Vol. 3 (Santa Cruz, CA: Lick Observatory)

Herbig, G. H., Petrov, P. P., \& Duemmler, R. 2003, ApJ, 595, 384

Herczeg, G. J., \& Hillenbrand, L. A. 2008, ApJ, 681, 594

Herczeg, G. J., \& Hillenbrand, L. A. 2014, ApJ, 786, 97

Hillenbrand, L. A., Carpenter, J. M., Kim, J. S., et al. 2008, ApJ, 677, 630

Hirth, G. A., Mundt, R., \& Solf, J. 1997, A\&AS, 126, 437

Hollenbach, D., \& Gorti, U. 2009, ApJ, 703, 1203

Hollenbach, D., \& McKee, C. F. 1979, ApJS, 41, 555

Hollenbach, D., \& McKee, C. F. 1989, ApJ, 342, 306

Howard, C. D., Sandell, G., Vacca, W. D., et al. 2013, ApJ, 776, 21

Hughes, A. M., Andrews, S. M., Wilner, D. J., et al. 2010, AJ, 140, 887

Husser, T.-O., Wende-von Berg, S., Dreizler, S., et al. 2013, A\&A, 553, A6

Jenkins, E. B. 2009, ApJ, 700, 1299

Jensen, E. L. N., Cohen, D. H., \& Gagné, M. 2009, ApJ, 703, 252

Johns-Krull, C. M., Valenti, J. A., \& Linsky, J. L. 2000, ApJ, 539, 815

Kennedy, G. M., \& Kenyon, S. J. 2009, ApJ, 695, 1210

Kenyon, S. J., Brown, D. I., Tout, C. A., \& Berlind, P. 1998, AJ, 115, 2491

Koester, D. 2010, MmSAI, 81, 921

Lavalley-Fouquet, C., Cabrit, S., \& Dougados, C. 2000, A\&A, 356, L41

Lazareff, B., Berger, J. P., Kluska, J., et al. 2017, A\&A, 599, A85

Lindegren, L., Hernandez, J., Bombrun, A., et al. 2018, A\&A, 616, A2

Loinard, L., Torres, R. M., Mioduszewski, A. J., \& Rodríguez, L. F. 2008, ApJL, 675, L29

Louvet, F., Dougados, C., Cabrit, S., et al. 2016, A\&A, 596, A88

Luhman, K. L., Allen, P. R., Espaillat, C., Hartmann, L., \& Calvet, N. 2010, ApJS, 186, 111

Luhman, K. L., \& Mamajek, E. E. 2012, ApJ, 758, 31

Luhman, K. L., Mamajek, E. E., Allen, P. R., \& Cruz, K. L. 2009, ApJ, 703, 399

Luhman, K. L., \& Rieke, G. H. 1999, ApJ, 525, 440

MacGregor, M. A., Wilner, D. J., Czekala, I., et al. 2017, ApJ, 835, 17

Manara, C. F., Frasca, A., Alcalá, J. M., et al. 2017, A\&A, 605, A86

Manara, C. F., Testi, L., Natta, A., et al. 2014, A\&A, 568, A18

Manara, C. F., Testi, L., Natta, A., \& Alcalá, J. M. 2015, A\&A, 579, A66

Matt, S., \& Pudritz, R. E. 2005, ApJL, 632, L135

Maurri, L., Bacciotti, F., Podio, L., et al. 2014, A\&A, 565, A110

McGinnis, P., Dougados, C., Alencar, S. H. P., Cabrit, S., \& Bouvier, J. 2018, arXiv: 1803.10287

Mohanty, S., Greaves, J., Mortlock, D., et al. 2013, ApJ, 773, 168

Najita, J. R., Andrews, S. M., \& Muzerolle, J. 2015, MNRAS, 450, 3559

Natta, A., Testi, L., Alcalá, J. M., et al. 2014, A\&A, 569, A5

Natta, A., Testi, L., \& Randich, S. 2006, A\&A, 452, 245

Nisini, B., Antoniucci, S., Alcalá, J. M., et al. 2018, A\&A, 609, A87

Pascucci, I., Edwards, S., Heyer, M., et al. 2015, ApJ, 814, 14

Pelletier, G., \& Pudritz, R. E. 1992, ApJ, 394, 117

Piétu, V., Guilloteau, S., Di Folco, E., Dutrey, A., \& Boehler, Y. 2014, A\&A, 564, A95

Pontoppidan, K. M., Blake, G. A., \& Smette, A. 2011, ApJ, 733, 84
Pudritz, R. E., Ouyed, R., Fendt, C., \& Brandenburg, A. 2007, in Protostars and Planets V, ed. B. Reipurth, D. Jewitt, \& K. Keil (Tucson, AZ: Univ. Arizona Press), 277

Pudritz, R. E., Rogers, C. S., \& Ouyed, R. 2006, MNRAS, 365, 1131

Pyo, T.-S., Hayashi, M., Kobayashi, N., et al. 2002, ApJ, 570, 724

Pyo, T.-S., Kobayashi, N., Hayashi, M., et al. 2003, ApJ, 590, 340

Ratzka, T., Köhler, R., \& Leinert, C. 2005, A\&A, 437, 611

Rebollido, I., Merín, B., Ribas, Á., et al. 2015, A\&A, 581, A30

Rebull, L. M., Padgett, D. L., McCabe, C.-E., et al. 2010, ApJS, 186, 259

Rice, E. L., Prato, L., \& McLean, I. S. 2006, ApJ, 647, 432

Rigliaco, E., Natta, A., Testi, L., et al. 2012, A\&A, 548, A56

Rigliaco, E., Pascucci, I., Gorti, U., Edwards, S., \& Hollenbach, D. 2013, ApJ, 772,60

Riviere-Marichalar, P., Merín, B., Kamp, I., Eiroa, C., \& Montesinos, B. 2016, A\&A, 594, A59

Riviere-Marichalar, P., Pinte, C., Barrado, D., et al. 2013, A\&A, 555, A67

Rodriguez, J. E., Pepper, J., Stassun, K. G., et al. 2015, AJ, 150, 32

Romanova, M. M., Ustyugova, G. V., Koldoba, A. V., \& Lovelace, R. V. E. 2009, MNRAS, 399, 1802

Safier, P. N. 1993, ApJ, 408, 115

Salyk, C., Herczeg, G. J., Brown, J. M., et al. 2013, ApJ, 769, 21

Sartori, M. J., Lépine, J. R. D., \& Dias, W. S. 2003, A\&A, 404, 913

Savage, B. D., \& Sembach, K. R. 1996, ARA\&A, 34, 279

Scicluna, P., Wolf, S., Ratzka, T., et al. 2016, MNRAS, 458, 2476

Shang, H., Li, Z.-Y., \& Hirano, N. 2007, in Protostars and Planets V, ed. B. Reipurth, D. Jewitt, \& K. Keil (Tucson, AZ: Univ. Arizona Press), 261 Shu, F. H. 1977, ApJ, 214, 488

Shull, J. M., \& McKee, C. F. 1979, ApJ, 227, 131

Sicilia-Aguilar, A., Henning, T., Kainulainen, J., \& Roccatagliata, V. 2011, ApJ, 736, 137

Sicilia-Aguilar, A., Henning, T., Linz, H., et al. 2013, A\&A, 551, A34

Simon, M., Guilloteau, S., Di Folco, E., et al. 2017, ApJ, 844, 158

Simon, M. N., Pascucci, I., Edwards, S., et al. 2016, ApJ, 831, 169

Skrutskie, M. F., Cutri, R. M., Stiening, R., et al. 2006, AJ, 131, 1163

Suzuki, T. K., \& Inutsuka, S.-i. 2009, ApJL, 691, L49

Takami, M., Chrysostomou, A., Ray, T. P., et al. 2004, A\&A, 416, 213

Tazzari, M., Testi, L., Natta, A., et al. 2017, A\&A, 606, A88

Tripathi, A., Andrews, S. M., Birnstiel, T., \& Wilner, D. J. 2017, ApJ, 845,44

van der Marel, N., Verhaar, B. W., van Terwisga, S., et al. 2016, A\&A, 592, A126

van der Marel, N., Williams, J. P., Ansdell, M., et al. 2018, ApJ, 854, 117

Varga, J., Ábrahám, P., Chen, L., et al. 2018, A\&A, 617, A83

Vogt, S. S., Allen, S. L., Bigelow, B. C., et al. 1994, Proc. SPIE, 2198, 362

Wahhaj, Z., Cieza, L., Koerner, D. W., et al. 2010, ApJ, 724, 835

Woitas, J., Ray, T. P., Bacciotti, F., Davis, C. J., \& Eislöffel, J. 2002, ApJ, 580,336

Wright, E. L., Eisenhardt, P. R. M., Mainzer, A. K., et al. 2010, AJ, 140, 1868

Zanni, C., \& Ferreira, J. 2009, A\&A, 508, 1117 